\title{
Aerodynamic Load Analysis of a Variable Camber Continuous Trailing Edge Flap System on a Flexible Wing Aircraft
}

\author{
Eric Ting* \\ Stinger Ghaffarian Technologies, Inc., Moffett Field, CA 94035 \\ Tung $\mathrm{Dao}^{\dagger}$ \\ Stinger Ghaffarian Technologies, Inc., Moffett Field, CA 94035 \\ Nhan Nguyen ${ }^{\ddagger}$ \\ NASA Ames Research Center, Moffett Field, CA 94035
}

\begin{abstract}
This paper presents an investigative aerodynamic analysis conducted on the novel control surface known as a Variable Camber Continuous Trailing Edge Flap (VCCTEF). The VCCTEF is modeled as a control effector on the NASA Generic Transport Model (GTM) where wing flexibility is considered. Aerodynamic modeling of the aircraft is conducted using vortex-lattice method (VLM), and an aeroelastic model of the aircraft that utilizes a coupled finite-element analysis (FEA) vortex-lattice solution is employed. VLM solutions are used to determine quasi-steady aerodynamic loading over the aeroelastic wing structures with VCCTEF. The load data is used to calculate aerodynamic sensitivities to control surface deployment and is also integrated to determine overall hinge moments. This analysis is conducted for different flight conditions, where control sensitivities compare the VCCTEF effectiveness against conventional control surfaces. Hinge moment results provide insight into aeroelastic wing loads, and worst case hinge moments for the VCCTEF can be estimated. Results show that the VCCTEF offers greater control authority than conventional ailerons at cruise, and nominal flap settings are determined for a low-speed take-off condition where the VCCTEF maintains comparable control effectiveness. Worst case hinge moment values are presented, where for a possible VCCTEF configuration, the stiff wing model demonstrates up to a $5.90 \%$ increase in flap hinge moment relative to a rigid model, and a reduced stiffness model demonstrates up to a $11.42 \%$ increase.
\end{abstract}

\section{Introduction}

With recent progress made in light-weight material technologies, the commercial aircraft industry has been investigating the design of lighter weight wing structures. These modern wing materials maintain the same load carrying capacity as conventional materials but with reduced structural rigidity. The advantage of using lighter weight materials is a desired direct reduction of aircraft weight, but the reduction in stiffness increases the aeroelastic interactions between flight aerodynamics and the wing structure. As a transition to these modern materials occurs, modeling capabilities for flexible wing aircraft becomes increasingly important.

In 2010, a conceptual study titled "Elastically Shaped Future Air Vehicle Concept"1 was conducted to investigate the benefits of several advanced aircraft technologies. The results demonstrated the potential benefits of using active control to tailor a flexible wing structure's aeroelastic shape during flight. To take advantage of the potential benefit, a novel control surface known as a Variable Camber Continuous Trailing Edge Flap (VCCTEF) system was proposed as a control surface candidate. ${ }^{1,2}$ Under the Fixed Wing Project Active Aeroelastic Shape Control (AASC) element, NASA and Boeing conducted joint studies to investigate the application of the VCCTEF system. ${ }^{3,4}$ The VCCTEF is implemented on the NASA Generic Transport Model (GTM), ${ }^{5}$ a notional model of short-to-medium range class commercial transport. The VCCTEF can be utilized to modify the wing's aeroelastic shape, allowing for optimization of the wing's spanwise twist at any point within the flight envelope to achieve a best lift-to-drag (L/D) ratio. This

\footnotetext{
*Engineer, Intelligent Systems Division, eric.b.ting@nasa.gov

${ }^{\dagger}$ Engineer, Intelligent Systems Division, tung.x.dao@ nasa.gov

${ }^{\ddagger}$ Research Scientist, Intelligent Systems Division, nhan.t.nguyen@ nasa.gov, AIAA Associate Fellow
} 
advantage is not conventionally possessed by commercial aircraft designs whose wings are twisted for a set cruise configuration and are not reshaped during flight. The VCCTEF also maintains a continuous trailing edge to avoid drag due to trailing edge discontinuities, generates lift more efficiently using continuous camber segments instead of a plain flap configuration, and also offers potential noise benefits.

Because the VCCTEF is a novel control surface, it is necessary to evaluate certain characteristics of the design. Given a VCCTEF physical specification, characterizing the loads on the control surface is necessary in order to gauge the efficacy of the system, estimate control deflections, and size the actuation system for the wing. The VCCTEF also possesses more moving parts than a conventional flap and aileron system, and the actuators for each of the sections and segments need to be sized based on the worst case hinge moments that the wing will experience. These types of analyses aim to provide insight into the VCCTEF system and its utility as a control surface to facilitate future detailed design considerations.

This paper describes a preliminary analysis of the aerodynamic loading on the GTM wing equipped with the VCCTEF. The analyses will be conducted for a rigid wing design with no flexibility, a baseline stiff wing design, and a flexible wing design. The flexible wing design is modeled with a reduction in stiffness of the baseline stiff wing values such that the flexible wing experiences tip deflection of about $10 \%$ of wing semi-span in flight. In order to determine the aeroelastic wing shapes at different flight conditions, a coupled vortex-lattice finite-element static aeroelasticity model that has been investigated in previous literature ${ }^{6,7}$ is utilized as needed for analysis of the VCCTEF.

Vortex-lattice method (VLM) ${ }^{8,9}$ is used as the primary aerodynamic modeling tool for analyzing sectional loads and is also run by the aeroelastic model. A geometry generation tool based upon the GTM geometry, jig-shape wing geometry, and VCCTEF configuration is used to create VLM input geometry. The load data can be used to determine VCCTEF control sensitivities as well as calculate hinge moments at the VCCTEF hinges. Previous studies have shown some preliminary estimates in obtaining this information through VLM, $, 4,10$ and this work explores the analysis framework and results in further detail and completeness. Aerodynamic hinge moment loads are evaluated for baseline steady flight conditions such as cruise, and control sensitivities are used to estimate a low-speed take-off condition as well as simulate coordinated turn maneuvers. Control sensitivities determined from VLM analysis for the VCCTEF are compared against the baseline GTM control surfaces, and an estimated worst-case aerodynamic hinge loading is determined.

\section{Generic Transport Model with Variable Camber Continuous Trailing Edge Flap System}

The NASA GTM (Generic Transport Model) is a sub-scale research platform representative of a classic commercial aircraft configuration. It includes a wind tunnel model and a remotely piloted vehicle, and the full-scale aircraft geometry is obtained by scaling up the sub-scale model by a ratio of 200:11. The GTM is modeled as a notional single-aisle, mid-size, 200-passenger aircraft representing one of the most common types of short-to-medium range passenger transport aircraft in the commercial aviation section. It has been tested within the 14-foot-by-22-foot wind tunnel test at NASA Langley Research Center and wind tunnel test data is available to validate computation models. Figure 1 is an illustration of the GTM aircraft. 


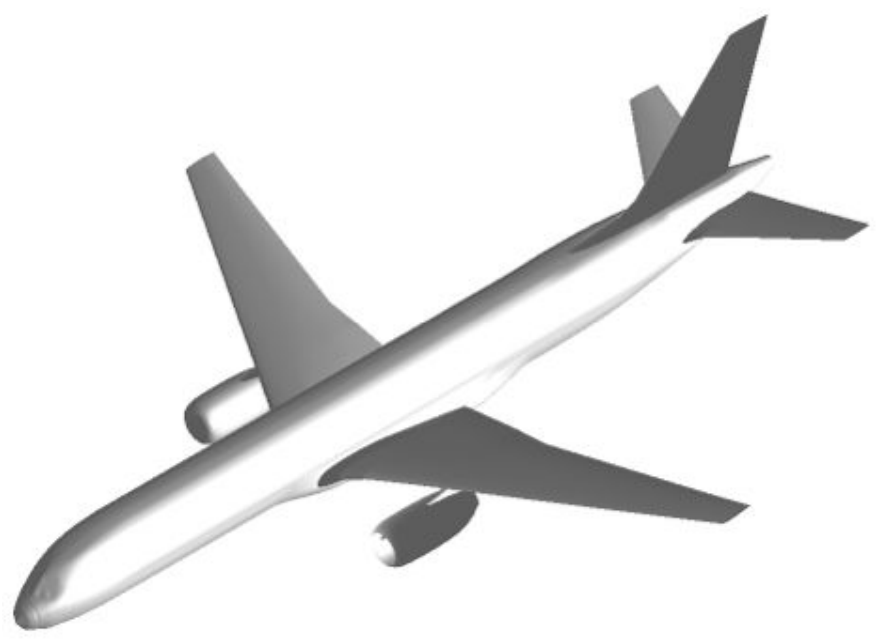

Figure 1. Illustration of GTM Aircraft

In the aeroelastic model of the GTM, the wing is allowed to freely deform based on reference wing stiffness values and the GTM jig-shape planform. A weight distribution model ${ }^{11}$ is used to determine the mass and inertia properties of the wing based on its operating condition. The "stiff" mass and stiffness model is representative of a commercial aircraft of the 757-era, similar in class to the GTM. ${ }^{4}$ A "flexible" mass and stiffness model is a notional wing model that is formulated by a $50 \%$ reduction of bending and torsion stiffnesses of the stiff wing. The flexible wing is designed to achieve a $10 \%$ wing tip deflection, mimicking the behavior of modern composite, high aspect ratio wing designs.

The GTM is equipped with the VCCTEF (Variable Camber Continuous Trailing Edge Flap) system as a wing shaping control and replacement for conventional ailerons and flaps. Details of the proposed VCCTEF concept are found in references. ${ }^{2-4}$ The VCCTEF is divided into a series of flap sections outboard of the wing break and one flap inboard of the wing break. Each of the flap sections are then composed of a series of camber segments that can be individually commanded. Figure 3 shows an example of an airfoil section with three camber segments.

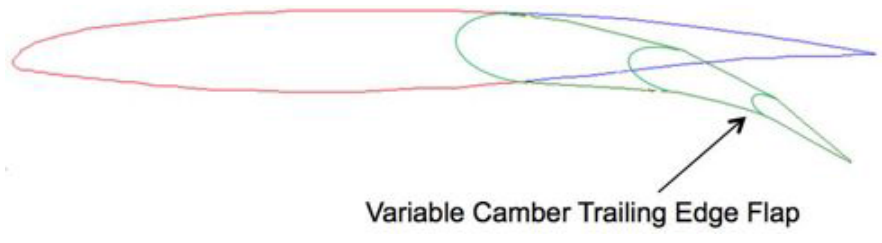

Figure 2. Variable Camber Flap

Two different configurations of VCCTEF will be examined in this study. Configuration \#1 represents a VCCTEF configuration where there are 15 outboard flap sections and three camber segments per flap section as shown in Fig. 3. Configuration \#2 represents a VCCTEF configuration where there are eight outboard flap sections and two camber segments per flap section as shown in Fig. 4. 


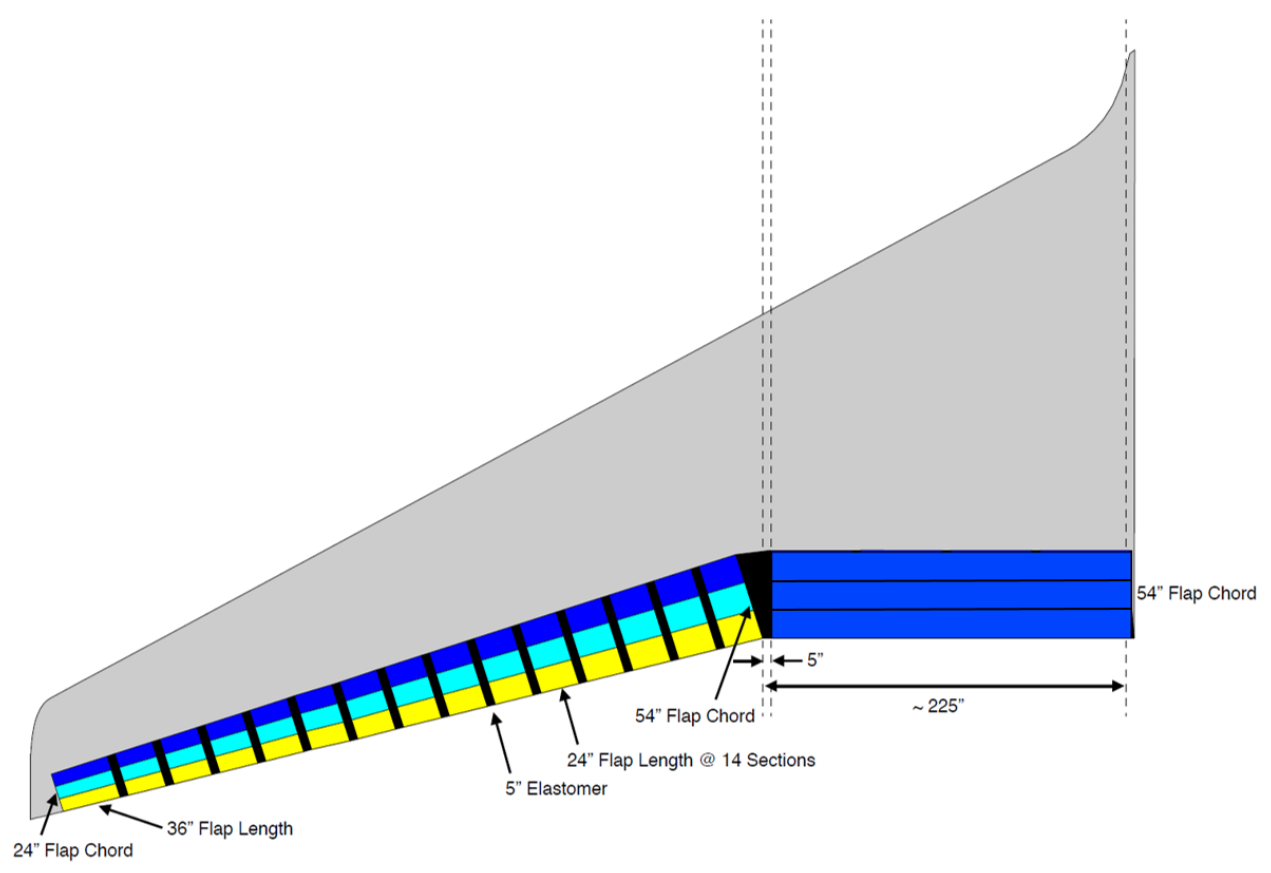

Figure 3. VCCTEF Configuration \#1

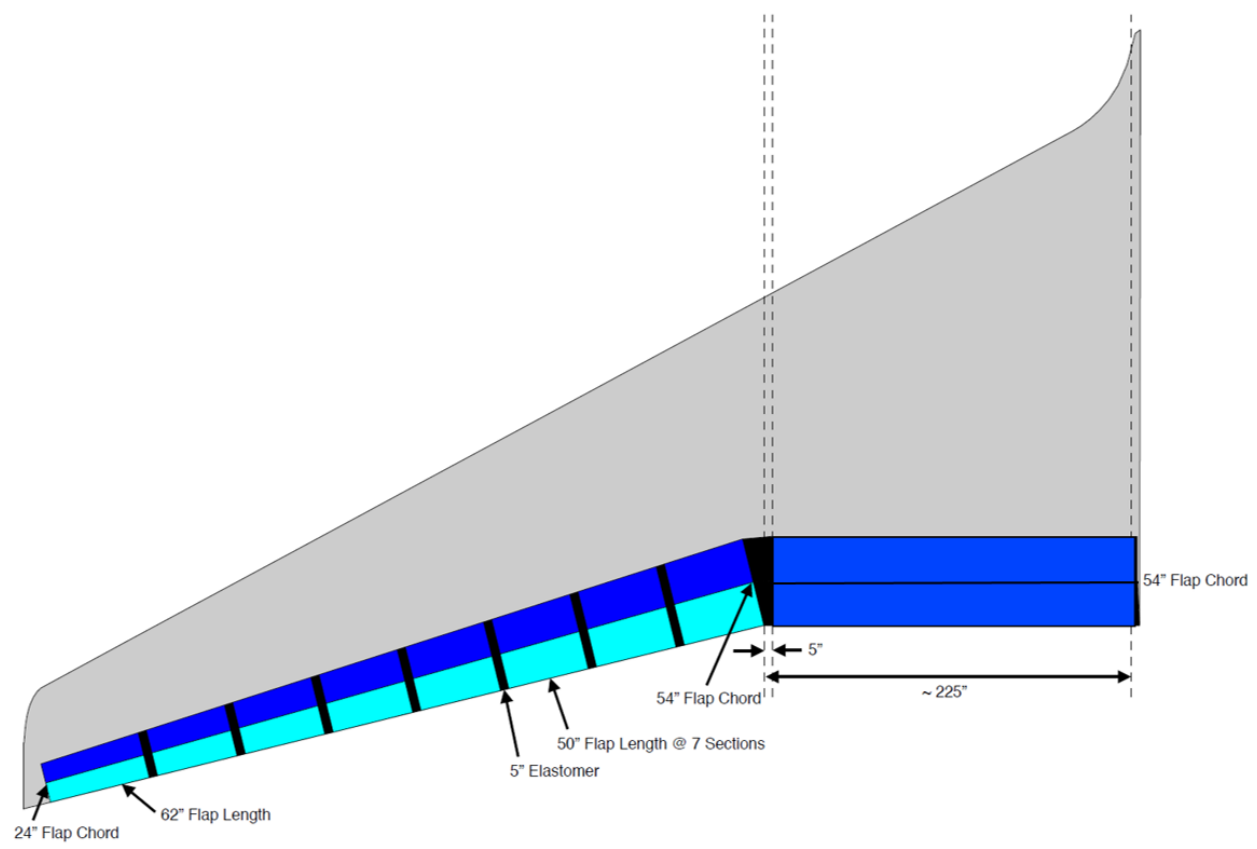

Figure 4. VCCTEF Configuration \#2

The variable camber flap sections are joined to the neighboring sections by a flexible and supported material which deforms and provides smooth transitions between flap sections without drag producing gaps. The relative flap deflections between any two adjacent spanwise flap sections are limited due to this flexible elastomer material, shown in black in Figs. 3 and 4. Figure 5 shows the VCCTEF configuration \#1 concept deployed on the GTM, where all three camber segments of the inboard flap are shown in light blue, the flexible material connecting neighboring flaps is shown in blue, and the deployed camber segments of the outboard flaps are shown in yellow, orange, and red for the 
first, second, and third camber segments of each flap, respectively.

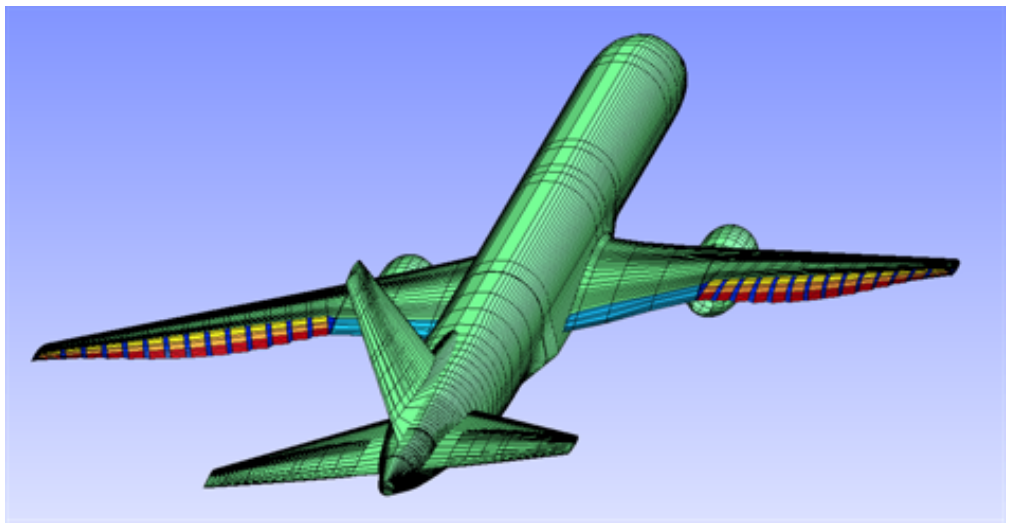

Figure 5. GTM with VCCTEF

For roll control and maneuvers, only the outermost camber segments of the outer flaps, which have a fast actuator response, are used. For VCCTEF configuration \#1, this represents the yellow 3rd camber segments in Fig. 3, and for VCCTEF configuration \#2, this represents the cyan 2nd camber segments in Fig. 4.

Let the flap deflection for a VCCTEF configuration be described as

$$
\delta=\left[\begin{array}{cccc}
\delta_{1}^{1} & \delta_{1}^{2} & \ldots & \delta_{1}^{m} \\
\vdots & \vdots & \ddots & \vdots \\
\delta_{n}^{1} & \delta_{n}^{2} & \ldots & \delta_{n}^{m}
\end{array}\right]
$$

where $m$ is the number of flap sections and $n$ is the number of camber segments per each of the flaps. For the scope of this study, the VCCTEF is always deployed using a circular camber arc. With a commanded flap deflection of $\Delta_{j}$ for the $j$-th flap section, the flap deflection on the $k$-th camber segment is $\delta_{k}^{j}=\frac{k \Delta_{j}}{n}$ where $n$ is the total number of camber segments. The shorthand $\delta=X^{\circ}$ where $X$ is a value in degrees is used to indicate the case where the all the flap sections are commanded with the same flap setting value $X$ and the camber segments follow the circular arc.

The aircraft has a mid-cruise weight of 210,000 lbs for a typical operating load (gear up, flap up) that includes cargo, fuel, and passengers. Fuel weight is about 75,000 lbs for a range of about 3,000 nautical miles. At the design cruise condition of Mach $=0.797$ at $36,000 \mathrm{ft}$, the design lift coefficient is $C_{L}=0.51$.

The aircraft's take-off gross weight (TOGW) is $235,000 \mathrm{lbs}$. At sea-level, this corresponds to a lift coefficient of $C_{L}=1.5614$ for 1 -g load. At take-off, the VCCTEF is deployed in a high-lift configuration (maximum deflection of $\delta=45^{\circ}$ ), and the initial concept of the VCCTEF includes the possibility of a slotted configuration. Since take-off is dominated by aerodynamic effects that would not be fully captured by the approach in this study, another "lowspeed take-off" flight condition is estimated where the aircraft has accelerated after lift-off and the VCCTEF has been retracted to a nominal scheduled setting. For the scope of this study, an airspeed of 150 knots is used as an estimate for the low-speed take-off flight condition, or Mach $=0.228$ at sea-level, similar to a previous study. ${ }^{4}$

\section{Vortex-Lattice Method}

VORVIEW is a computational tool used for aerodynamic modeling of aircraft configurations using the VORLAX vortex-lattice method code. ${ }^{9}$ Based on lifting line/vortex-lattice aerodynamic theory, VORVIEW/VORLAX provides a rapid method for estimating aerodynamic force and moment coefficients. Geometric input vehicle configurations are constructed within VORVIEW by discretizing the surface into a series of panels, and each panel is then represented by the placement of spanwise and chordwise locations of bound or horseshoe vortices. Vortex-panels model lifting geometry as mean camber surfaces.

VORLAX is considered a medium-fidelity tool, and limitations associated with vortex-lattice modeling in general apply to VORVIEW aerodynamic analysis. Vortex-lattice is based on potential flow theory for inviscid and incompressible flow, where VORLAX accounts for compressibility effects on the aerodynamic coefficients through a 
compressibility correction, such as the Prandtl-Glauert correction. Due to its inviscid nature, the drag prediction by VORLAX is most reliable only for induced drag. Prediction of viscous drag due to boundary layer separation and wave drag due to shock-induced boundary layer separation are generally not conducted by vortex-lattice, and viscous drag must be estimated using other methods.

For lift prediction at low angle of attacks, VORLAX can provide reasonably accurate prediction. VORVIEW has been validated by both wind tunnel data ${ }^{5}$ as well as the NASA Cart3D tool, ${ }^{12}$ a high-fidelity inviscid (Euler) CFD analysis code. Agreement between VORVIEW, Cart3D, and wind tunnel results have been shown for the GTM platform. ${ }^{1,2,13}$ In general, both VORVIEW and Cart3D seem to have similar predictive capabilities when compressibility is not a factor.

\section{A. Vortex-Lattice Configuration}

For this particular study, the engines and nacelles of the GTM are not included in the vortex-lattice model as a simplification, since they are not considered lifting surfaces. ${ }^{21}$ Figure 6 represents the input geometry of the GTM (VCCTEF configuration \#1 with no engine nacelles and pylons) in VORVIEW as well as the particular vortex-lattice panel/polygon configuration used to examine the aircraft total aerodynamics.

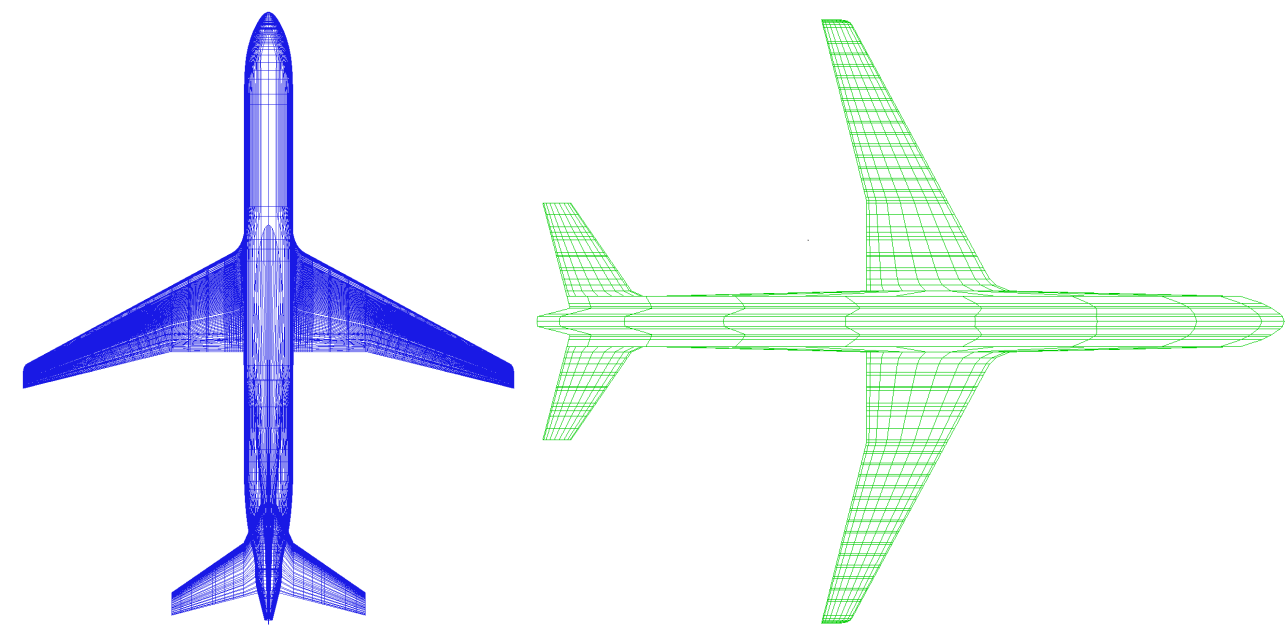

Figure 6. GTM Vortex-Lattice Geometry and Panel/Polygon Configuration

The panel discretization is identical to the spanwise polygon discretization validated and used for the GTM in a previous study ${ }^{13}$ except with the inclusion of the tail empennage. The vortex-lattice has panel/polygon edges placed along the trailing edge locations of the boundaries of the VCCTEF sections to ensure that deflection of the VCCTEF is fully captured within vortex-lattice analysis. This vortex-lattice configuration is used in the static aeroelastic modeling of the GTM.

A more refined vortex-lattice configuration is used for the control derivative portion of this study. This is done to increase the resolution of the spanwise sectional aerodynamic coefficients for derivative calculations. The fine vortex-lattice panel/polygon configurations used are shown in Fig. 7. 

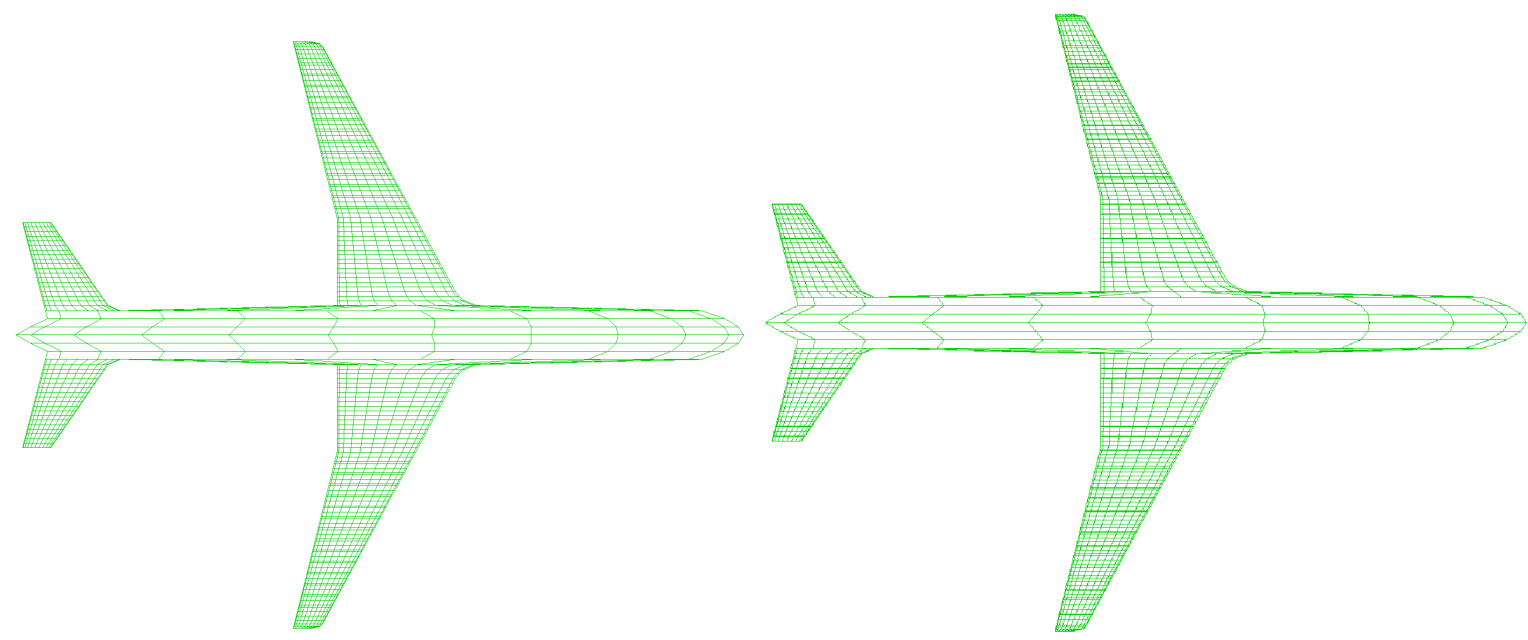

Figure 7. GTM Fine Vortex-Lattice Panel/Polygon Configuration for Control Derivative Analysis (VCCTEF configuration \#1, left, VCCTEF configuration \#2, right)

All configurations are run in vortex-lattice using a symmetric flight assumption where there is no aircraft sideslip angle, $\beta=0^{\circ}$, and the aircraft wing spanwise axis, or pitch axis, is the same as the aircraft stability or velocity axes. In addition, only symmetric geometries are currently utilized.

\section{B. Automated Geometry Generation Tool for Vortex-Lattice}

An automated geometry generation tool is used to generate the input geometry into VORVIEW. The geometry generation tool uses a global axis system with the origin fixed at the aircraft nose. Let this global coordinate system be represented by a coordinate system $[x, y, z]$. The $x$-axis starts at the aircraft nose and points directly aft towards the tail, the $y$-axis runs outboard towards the tip of the right wing, and the $z$-axis runs vertically upwards as shown in Fig. 8 .

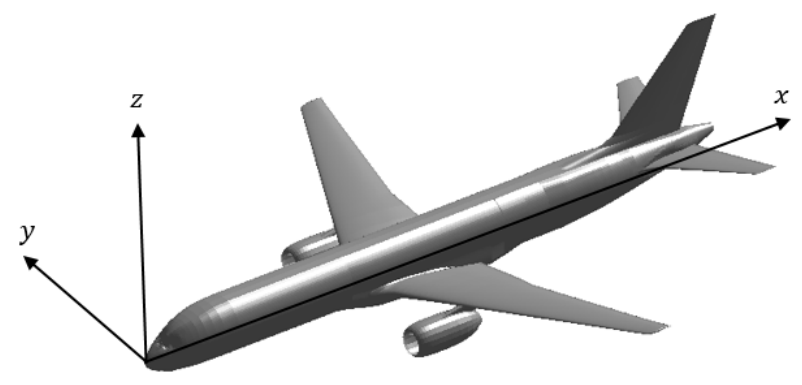

Figure 8. Global Coordinate System

Given a wing elastic axis defined in the global axis system $\left(x_{e a}, y_{e a}, z_{e a}\right)$, the geometry generation tool is able to automatically generate an aeroelastically deformed mesh for input into VORVIEW given a structural deformation (generally determined from FEA solution). Let the structural deformation of an aeroelastic wing be described by three translations and three rotations, represented by the vectors:

$$
\begin{gathered}
\Delta \mathbf{r}=\Delta x \hat{\mathbf{i}}+\Delta y \hat{\mathbf{j}}+\Delta z \hat{\mathbf{k}} \\
\Delta \phi=\Delta \phi_{x} \hat{\mathbf{i}}+\Delta \phi_{y} \hat{\mathbf{j}}+\Delta \phi_{z} \hat{\mathbf{k}}
\end{gathered}
$$

where $\hat{\mathbf{i}}, \hat{\mathbf{j}}$, and $\hat{\mathbf{k}}$ are unit vectors in the directions of the $x-, y$-, and $z$-global directions.

The geometry generation tool has access to the outer mold line of the jig-shape (undeformed) wing geometry. Let a point on the on the outer mold of the wing be represented by $\left(x_{w}, y_{w}, z_{w}\right)$. The geometry generation tool applies the vectors $\Delta \mathbf{r}$ and $\Delta \phi$ through the following transformation: 


$$
\begin{aligned}
& \left\{\begin{array}{c}
\tilde{x}_{w} \\
\tilde{y}_{w} \\
\tilde{z}_{w}
\end{array}\right\}=\left[\begin{array}{ccc}
\cos \Delta \phi_{z} & -\sin \Delta \phi_{z} & 0 \\
\sin \Delta \phi_{z} & \cos \Delta \phi_{z} & 0 \\
0 & 0 & 1
\end{array}\right]\left[\begin{array}{ccc}
1 & 0 & 0 \\
0 & \cos \Delta \phi_{x} & -\sin \Delta \phi_{x} \\
0 & \sin \Delta \phi_{x} & \cos \Delta \phi_{x}
\end{array}\right]\left[\begin{array}{ccc}
\cos \Delta \phi_{y} & 0 & \sin \Delta \phi_{y} \\
0 & 1 & 0 \\
-\sin \Delta \phi_{y} & 0 & \cos \Delta \phi_{y}
\end{array}\right]\left\{\begin{array}{c}
x_{w}-x_{e a}\left(y_{w}\right) \\
0 \\
z_{w}-z_{e a}\left(y_{w}\right)
\end{array}\right\} \\
& +\left\{\begin{array}{c}
x_{e a}\left(y_{w}\right)+\Delta x \\
y_{w}+\Delta y \\
z_{e a}\left(y_{w}\right)+\Delta z
\end{array}\right\}
\end{aligned}
$$

where $\tilde{x}_{w}, \tilde{y}_{w}$, and $\tilde{z}_{w}$ is the location of the deformed outer mold point.

The geometry generation tool is also capable of deflecting the VCCTEF by rotating the corresponding flap section of an airfoil section about a hinge line. Examples of the geometry with the VCCTEF deflection are shown in Fig. 9.
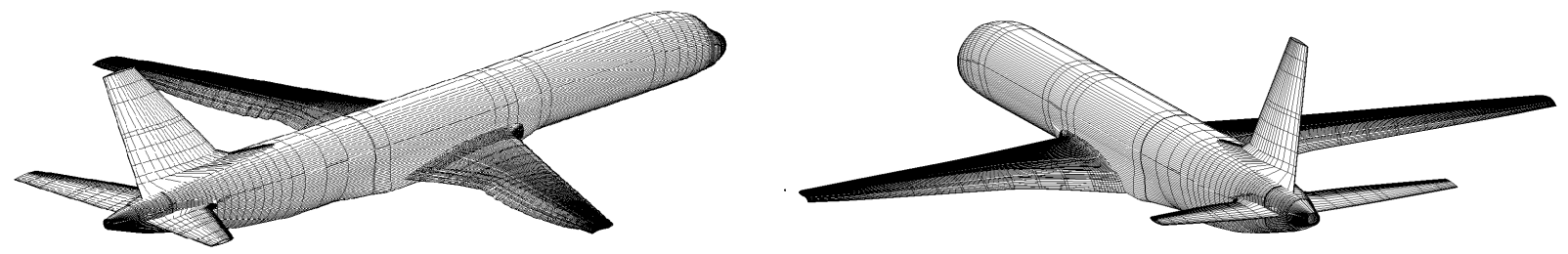

Figure 9. Example VCCTEF Deflected Wing Geometry

The geometry generation tool returns the hinge line locations for the VCCTEF as the aircraft geometry is generated. For each camber segment, there is a corresponding set data $\left(x_{h_{k}}, y_{h_{k}}, z_{h_{k}}\right)$ which represents the axis of rotation for the $k$-th camber segments of the VCCTEF. The hinge lines are approximated to coincide with the location of the mean camber line of the wing's airfoil sections.

\section{VORLAX Panel Results}

Vortex-lattice returns the aerodynamic characteristics for each of the vortex-panels in the input configuration. The data returned for each vortex-panel can be post-processed and utilized to conduct further analysis.

\section{Coordinate System}

Each vortex-panel has its own defining geometry, and it is useful to define several quantities for analysis. Let an arbitrary vortex-panel be considered. A panel coordinate frame is formed represented by $[\mu, \nu, \xi]$ where the $\mu$-axis points along the mean panel centerline. The $v$-axis lies perpendicular to the $\mu$-axis and along the panel's leading edge. The $\xi$-axis forms a right handed rectangular coordinate axis with the $\mu$-axis and the $v$-axis. A vortex-panel coordinate frame is shown in Fig. 10.

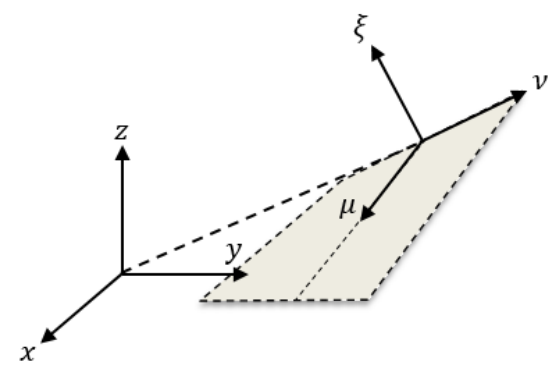

Figure 10. Arbitrary Vortex-Panel Coordinate System 
Coordinate transformations can be used to relate vectors in the global coordinate to the panel coordinate system. Starting from the global frame directions, the first coordinate rotation is about the $x$-axis by a value $\Gamma$, or the panel's dihedral at the leading edge. This locates an intermediate coordinate frame $\left[x^{\prime}, y^{\prime}, z^{\prime}\right]$. A second coordinate rotation then rotates the the intermediate coordinate frame about the $y^{\prime}$-axis by the angle $\gamma$, the panel's incidence (VORLAX convention defines incidence as positive nose-down). Figure 11 shows the coordinate transformations from the global axes directions, to the intermediate coordinate frame, and to an arbitrary panel frame.
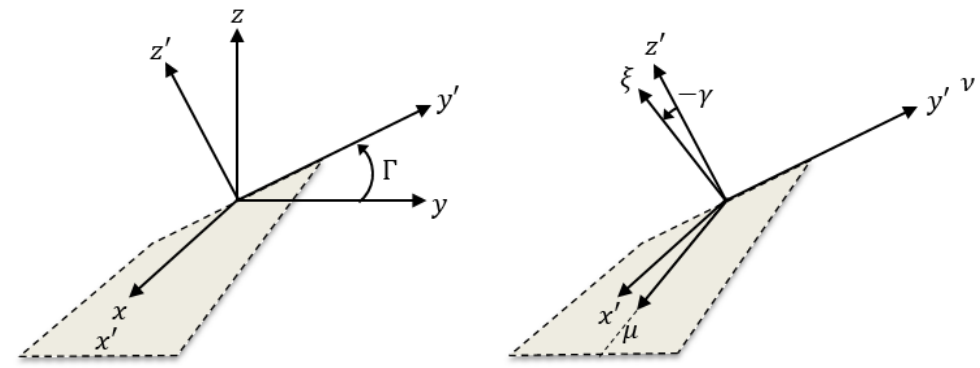

Figure 11. Coordinate Rotations Between Global Axes Directions to Vortex-Panel Frame

The coordinate transformations can be expressed mathematically as

$$
\left[\begin{array}{l}
\mu \\
\nu \\
\xi
\end{array}\right]=\left[\begin{array}{ccc}
\cos \gamma & 0 & \sin \gamma \\
0 & 1 & 0 \\
-\sin \gamma & 0 & \cos \gamma
\end{array}\right]\left[\begin{array}{ccc}
1 & 0 & 0 \\
0 & \cos \Gamma & \sin \Gamma \\
0 & -\sin \Gamma & \cos \Gamma
\end{array}\right]\left[\begin{array}{l}
x \\
y \\
z
\end{array}\right]
$$

Let a vortex-lattice configuration consist of a total of $n_{p}$ panels. Henceforth, the superscript $i$ is used to represent the vortex-panel index. For the $i$-th panel, a coordinate transformation relationship can be established that transforms vectors in the $i$-th vortex-panel to their equivalent representation in the global axes frame.

$$
T^{i}=\left[\begin{array}{ccc}
1 & 0 & 0 \\
0 & \cos \Gamma^{i} & -\sin \Gamma^{i} \\
0 & \sin \Gamma^{i} & \cos \Gamma^{i}
\end{array}\right]\left[\begin{array}{ccc}
\cos \gamma^{i} & 0 & -\sin \gamma^{i} \\
0 & 1 & 0 \\
\sin \gamma^{i} & 0 & \cos \gamma^{i}
\end{array}\right]=\left[\begin{array}{ccc}
\cos \gamma^{i} & 0 & -\sin \gamma^{i} \\
-\sin \gamma^{i} \sin \Gamma^{i} & \cos \Gamma^{i} & -\cos \gamma^{i} \sin \Gamma^{i} \\
\sin \gamma^{i} \cos \Gamma^{i} & \sin \Gamma^{i} & \cos \gamma^{i} \cos \Gamma^{i}
\end{array}\right]
$$

\section{Panel Sectional Aerodynamic Coefficients}

For each $i$-th vortex panel, VORVIEW/VORLAX returns the $\Delta c_{P}$ values as well as the integrated $c_{N}$ (panel normal force coefficient), $c_{D}$ (panel drag force coefficient), and integrated $c_{M_{\frac{c}{4}}}$ (panel pitching moment coefficient about the local quarter-chord). Note that VORLAX returns $\Delta c_{P}$ values as a function of $\frac{x}{c^{i}}$, where $x$ is in the global axis direction and vortex-lattice assumes that the panel chord is constant and equal to the value of the panel centerline, $c^{i}$.

$$
\begin{aligned}
\Delta c_{P}^{i}\left(\frac{x}{c^{i}}\right)=\frac{1}{S^{i}} \int_{v_{1}}^{v_{2}}\left(\frac{p_{\text {lower }}^{i}\left(\frac{x}{c^{i}}\right)-p_{\text {upper }}^{i}\left(\frac{x}{c^{i}}\right)}{q_{\infty}}\right) d v \\
c_{N}^{i}=\frac{N^{i}}{q_{\infty} S^{i}} \\
c_{D}^{i}=\frac{D^{i}}{q_{\infty} S^{i}} \\
c_{M_{\frac{c}{4}}^{i}}^{i}=\frac{M_{\frac{c}{4}}^{i}}{q_{\infty} S^{i} c^{i}}
\end{aligned}
$$


where $S^{i}$ is the $i$-th panel's surface area. Converting into sectional values involves changing the spanwise variable to the spanwise aircraft $y$-axis where $y_{1}^{i}$ and $y_{2}^{i}$ are the panel edges where, by design, $y_{2}^{i}>y_{1}^{i}$.

$$
\begin{gathered}
\Delta c_{p}(y)=\frac{1}{q_{\infty} S^{i}} \int_{y_{1}}^{y_{2}}\left(p_{\text {lower }}^{i}(\mu)-p_{\text {upper }}^{i}(\mu)\right) \frac{d y}{\cos \Gamma^{i}}=\Delta c_{P}^{i}(\mu) \frac{1}{\cos \Gamma^{i}} \\
c_{n}^{i}=\frac{N^{i}}{q_{\infty} c^{i} b^{i}} \frac{b^{i}}{\left(y_{2}^{i}-y_{1}^{i}\right)}=\frac{N^{i}}{q_{\infty} S^{i}} \frac{1}{\cos \Gamma^{i}}=c_{N}^{i} \frac{1}{\cos \Gamma^{i}} \\
c_{d}^{i}=\frac{D^{i}}{q_{\infty} c^{i} b^{i}} \frac{b^{i}}{\left(y_{2}^{i}-y_{1}^{i}\right)}=\frac{D^{i}}{q_{\infty} S^{i}} \frac{1}{\cos \Gamma^{i}}=c_{D}^{i} \frac{1}{\cos \Gamma^{i}} \\
c_{m_{\frac{c}{4}}}^{i}=\frac{M_{\frac{c}{4}}^{i}}{q_{\infty} c^{i} b^{i} c^{i}} \frac{b^{i}}{\left(y_{2}^{i}-y_{1}^{i}\right)}=\frac{M_{\frac{c}{4}}^{i}}{q_{\infty} S^{i} c^{i}} \frac{1}{\cos \Gamma^{i}}=c_{M_{\frac{c}{4}}}^{i} \frac{1}{\cos \Gamma^{i}}
\end{gathered}
$$

where $b^{i}$ is the span of the $i$-th panel in its local frame and $\Gamma^{i}$ is the dihedral. These sectional panel coefficients are equivalent to $c_{n}\left(y^{i}\right), c_{d}\left(y^{i}\right)$ and $c_{m_{\frac{c}{4}}}\left(y^{i}\right)$ where $y^{i}$ is the location of the panel centerline. Combining the data from all $n_{p}$ panels produces the spanwise distributions $c_{n}(y), c_{d}(y)$ and $c_{m_{\frac{c}{4}}}(y)$.

\section{Static Aeroelastic Model}

A static aeroelastic model is used in this study in order to predict the aeroelastic wing shape and aerodynamics for the GTM model with VCCTEF. A coupled vortex-lattice finite-element static aeroelastic model is used, and the framework is identical to that used in previous studies. ${ }^{7,13}$ Figure 12 represents the coupled aero-structural iterations conducted by the coupled vortex-lattice finite-element static aeroelastic code. Details for the formulation of the finiteelement model used in the static aeroelastic model are found in references. ${ }^{7,13}$

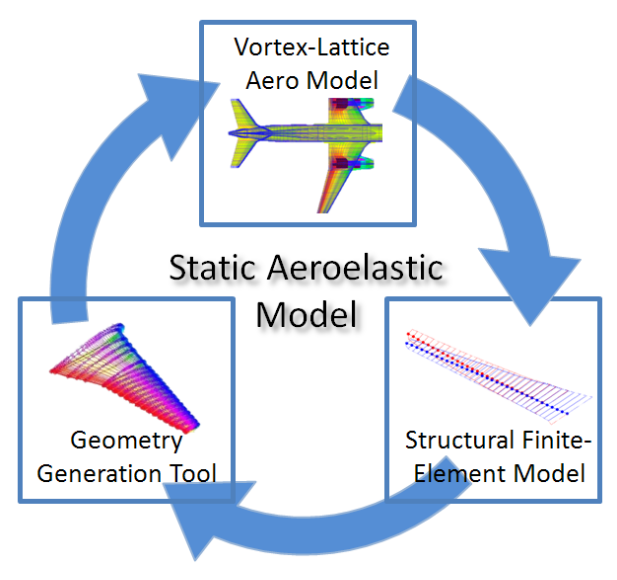

Figure 12. Static Aeroelastic Model Concept

The static aeroelastic code maps an input flight condition corresponding to a lift coefficient $C_{L}$, Mach number $M$, and altitude $h$ into the respective static aeroelastic deflection solution a flexible wing would experience at that flight condition and the angle of attack $\alpha$ that the flexible wing aircraft would experience when leveled at the desired $C_{L}$. Currently, no engine thrust is considered. The following procedure is followed:

1. Vortex-lattice modeling is conducted on an input geometry at the specified flight condition to determine the aircraft total aerodynamic quantities, as well as sectional coefficients $c_{l}(y), c_{m_{a c}}(y)$, and $x_{a c}(y)$ or the location of the section aerodynamic centers.

2. The structural FEM model uses the sectional aerodynamic load inputs to calculate the wing's structural deflection. These are represented as an elastic axis twist $\Theta(y)$ and vertical tip deflection $W(y)$. 
3. The FEM solution is converted into deformation vectors $\Delta \mathbf{r}$ and $\Delta \phi$, which are input into the geometry generation tool. The automated geometry tool generates a new aircraft geometry with the deformed wing.

4. A lift curve is generated based on the deformed wing aircraft geometry. The angle of attack $\alpha$ for the value $C_{L}$ is determined and selected for the next iteration.

5. Steps 1-4 are repeated until $|\Delta \alpha|$ between iterations is within a criteria.

This static aeroelastic model outputs $\Theta(y), W(y), W_{x}(y)$, which describe the aeroelastic deflection of the wing.

\section{A. Cruise Condition}

At the GTM's design cruise condition, the static aeroelastic solutions for the model and configuration used in this study are summarized in Table 1. Though the geometry at cruise is identical with both VCCTEF configurations, the slight difference in static aeroelastic solution is due to the difference in the vortex-lattice polygon/panel configuration used to analyze each model. At the cruise condition, the VCCTEF is completely stowed. The values of $\Theta$ and $W$ at the wing tip are returned, as well as the value $\Delta \phi_{y}$ from Eq. 3, or the twist of the wing section about the pitch axis.

\begin{tabular}{|c|c|c|c|c|}
\hline \multicolumn{5}{|c|}{ VCCTEF Configuration \#1 } \\
\hline & $\alpha(\mathrm{deg})$ & $W_{\text {tip }}(\mathrm{ft})$ & $\Theta_{\text {tip }}(\mathrm{deg})$ & $\Delta \phi_{y, \text { tip }}(\mathrm{deg})$ \\
\hline \hline Rigid Wing & 2.792 & - & - & - \\
\hline Stiff Wing & 3.237 & 2.053 & -0.303 & -1.206 \\
\hline Flexible Wing & 3.679 & 3.826 & -0.294 & -2.470 \\
\hline
\end{tabular}

\begin{tabular}{|c|c|c|c|c|}
\hline \multicolumn{5}{|c|}{ VCCTEF Configuration \#2 } \\
\hline & $\alpha(\mathrm{deg})$ & $W_{\text {tip }}(\mathrm{ft})$ & $\Theta_{\text {tip }}(\mathrm{deg})$ & $\Delta \phi_{y, t i p}(\mathrm{deg})$ \\
\hline \hline Rigid Wing & 2.787 & - & - & - \\
\hline Stiff Wing & 3.245 & 2.086 & -0.288 & -1.257 \\
\hline Flexible Wing & 3.699 & 3.889 & -0.269 & -2.566 \\
\hline
\end{tabular}

Table 1. Static Aeroelastic Solutions for 1-g Steady State Cruise, $M=0.797, h=36000 \mathbf{f t}, C_{L}=0.51$

\section{B. Low-Speed Take-Off Condition}

For the GTM's low-speed take-off flight condition, static aeroelastic solutions are determined and summarized in Table 2. For the two VCCTEF configurations, a nominal VCCTEF flap deflection for augmented lift was selected. For VCCTEF configuration \#1, the VCCTEF deflection was set at $\delta=25^{\circ}$, and for VCCTEF configuration \#2, the nominal VCCTEF deflection was set at $\delta=35^{\circ}$. 


\begin{tabular}{|c|c|c|c|c|}
\hline \multicolumn{5}{|c|}{ VCCTEF Configuration \#1 } \\
\hline$\delta=25^{\circ}$ & $\alpha(\mathrm{deg})$ & $W_{\text {tip }}(\mathrm{ft})$ & $\Theta_{\text {tip }}(\mathrm{deg})$ & $\Delta \phi_{y, t i p}(\mathrm{deg})$ \\
\hline \hline Rigid Wing & 7.977 & - & - & - \\
\hline Stiff Wing & 8.541 & 2.436 & -0.365 & -1.472 \\
\hline Flexible Wing & 9.208 & 4.866 & -0.631 & -3.012 \\
\hline
\end{tabular}

\begin{tabular}{|c|c|c|c|c|}
\hline \multicolumn{5}{|c|}{ VCCTEF Configuration \#2 } \\
\hline$\delta=35^{\circ}$ & $\alpha(\mathrm{deg})$ & $W_{\text {tip }}(\mathrm{ft})$ & $\Theta_{\text {tip }}(\mathrm{deg})$ & $\Delta \phi_{y, t i p}(\mathrm{deg})$ \\
\hline \hline Rigid Wing & 4.417 & - & - & - \\
\hline Stiff Wing & 5.004 & 2.781 & -0.111 & -1.993 \\
\hline Flexible Wing & 5.740 & 5.498 & -0.114 & -3.994 \\
\hline
\end{tabular}

Table 2. Static Aeroelastic Solutions for 1-g Steady State Low-Speed Take-Off, $M=0.228, h=0$ ft, $C_{L}=1.56$

\section{Control Derivative Analysis}

A control sensitivity analysis based upon the geometric configuration of a VCCTEF design is able to gauge the control efficacy of a given flap configuration. Control derivatives can be directly compared against the values for the conventional flap and aileron systems on the GTM, or estimate the amount of flap deflection is needed in order conduct maneuvers. VORVIEW vortex-lattice modeling with the VORLAX engine and the accompanying automated geometry generation tool are used to conduct the aerodynamic modeling needed to evaluate the control sensitivities.

\section{A. Methodology}

Based on the definition of $\delta$ in Eq. 1, for the first VCCTEF configuration $m=16$ and $n=3$, while for the second VCCTEF configuration $m=9$ and $n=2$. Let $\bar{\delta}$ be a known nominal flap setting for the aircraft at a point in the flight envelope. For the mid-cruise condition the VCCTEF is completely stowed and $\bar{\delta}$ is the zero matrix or via shorthand, $\bar{\delta}=0^{\circ}$.

For each $j$-th flap section, vortex-lattice is run for three flap inputs: the nominal flap setting $\bar{\delta}$, and two flap settings where the aftmost camber segment (or $n$-th camber segment) of the $j$-th section is perturbed by a small value $\pm \varepsilon$. In vortex-lattice modeling, the approach in this study is to always deploy the VCCTEF symmetrically and to examine the right wing results for simplification. The left wing aerodynamics are determined through reflection.

\section{Wing Sectional Derivatives}

The sectional normal force coefficients $c_{n}(y)$ are transformed into the global axes coefficients using the transformation in Eq. 6.

$$
\left[\begin{array}{l}
c_{x} \\
c_{y} \\
c_{z}
\end{array}\right]=\left[\begin{array}{ccc}
\cos \gamma & 0 & -\sin \gamma \\
-\sin \gamma \sin \Gamma & \cos \Gamma & -\cos \gamma \sin \Gamma \\
\sin \gamma \cos \Gamma & \sin \Gamma & \cos \gamma \cos \Gamma
\end{array}\right]\left[\begin{array}{c}
c_{a} \\
0 \\
c_{n}
\end{array}\right]=\left[\begin{array}{c}
-c_{n} \sin \gamma+c_{a} \cos \gamma \\
-c_{n} \cos \gamma \sin \Gamma-c_{a} \sin \gamma \sin \Gamma \\
c_{n} \cos \gamma \cos \Gamma+c_{a} \sin \gamma \cos \Gamma
\end{array}\right]
$$

Considering symmetric flight with no sideslip angle $\beta$, the sectional lift and drag coefficients, $c_{l}(y)$ and $c_{d}(y)$, can be determined using the aircraft angle of attack $\alpha$.

$$
\left[\begin{array}{l}
c_{l} \\
c_{d}
\end{array}\right]=\left[\begin{array}{cc}
\cos \alpha & -\sin \alpha \\
\sin \alpha & \cos \alpha
\end{array}\right]\left[\begin{array}{l}
c_{z} \\
c_{x}
\end{array}\right]
$$


Since VORLAX returns $c_{n}$ and $c_{d}$, the axial force coefficient can be solved through

$$
\begin{aligned}
& c_{d}= {\left[\begin{array}{ccc}
-\sin \alpha & 0 & \cos \alpha \\
0 & 1 & 0 \\
\cos \alpha & 0 & \sin \alpha
\end{array}\right]\left[\begin{array}{c}
-c_{n} \sin \gamma+c_{a} \cos \gamma \\
-c_{n} \cos \gamma \sin \Gamma-c_{a} \sin \gamma \sin \Gamma \\
c_{n} \cos \gamma \cos \Gamma+c_{a} \sin \gamma \cos \Gamma
\end{array}\right] } \\
&=\left(-c_{n} \sin \gamma+c_{a} \cos \gamma\right) \cos \alpha+\left(c_{n} \cos \gamma \cos \Gamma+c_{a} \sin \gamma \cos \Gamma\right) \sin \alpha \\
&= c_{n}(-\sin \gamma \cos \alpha+\cos \gamma \cos \Gamma \sin \alpha)+c_{a}(\sin \gamma \cos \Gamma \sin \alpha+\cos \gamma \cos \alpha) \\
& c_{a}=\frac{c_{d}-c_{n}(\cos \gamma \cos \Gamma \sin \alpha-\sin \gamma \cos \alpha)}{\sin \gamma \cos \Gamma \sin \alpha+\cos \gamma \cos \alpha}
\end{aligned}
$$

Thus the sectional aircraft spanwise force coefficients are summarized in Eqs. 21-24, where a parasite drag term $c_{d_{0}}(y)$ is included. A skin friction model using a flat plate approach is used to estimate $c_{d_{0}}$.

$$
\begin{gathered}
c_{x}(y)=-c_{n}(y) \sin \gamma(y)+c_{a}(y) \cos \gamma(y)+c_{d_{0}}(y) \cos \alpha \\
c_{y}(y)=-c_{n}(y) \cos \gamma(y) \sin \Gamma(y)-c_{a}(y) \sin \gamma(y) \sin \Gamma(y) \\
c_{z}(y)=c_{n}(y) \cos \gamma(y) \cos \Gamma(y)+c_{a}(y) \sin \gamma(y) \cos \Gamma(y)+c_{d_{0}}(y) \sin \alpha \\
c_{l}(y)=c_{n}(y) \cos \gamma(y) \cos \Gamma(y) \cos \alpha+c_{a}(y) \sin \gamma(y) \cos \Gamma(y) \cos \alpha+c_{n}(y) \sin \gamma(y) \sin \alpha-c_{a}(y) \cos \gamma(y) \sin \alpha
\end{gathered}
$$

Vortex-lattice returns the sectional pitching moment about the panel quarter chords, $c_{m_{\frac{c}{4}}}(y)$. The location of the panel quarter chords is represented by $x_{\frac{c}{4}}(y)$ and $z_{\frac{c}{4}}(y)$ for a spanwise wing station $y$. Since the aircraft's elastic axis is known, the pitching moment coefficient about the local elastic axis can be calculated as

$c_{m_{e a}}(y)=\left(c_{m_{\frac{c}{4}}}(y) \cos \Gamma(y)+c_{z}(y) \frac{x_{e a}(y)-x_{\frac{c}{4}}(y)}{c(y)}-c_{x}(y) \frac{z_{e a}(y)-z(y)}{c(y)}\right) \cos \Gamma_{e a} \cos \Lambda_{e a}+c_{m_{\frac{c}{4}}}(y) \sin \Gamma(y) \sin \Gamma_{e a} \cos \Lambda_{e a}$

where $\Lambda_{e a}$ and $\Gamma_{e a}$ are the sweep and dihedral angles of the wing's equivalent elastic axis. If the elastic axis dihedral is small, then the values of $c_{m_{e a}}$ can be approximated as

$$
c_{m_{e a}}(y)=\left(c_{m_{\frac{c}{4}}}(y) \cos \Gamma(y)+c_{z}(y) \frac{x_{e a}(y)-x_{\frac{c}{4}}(y)}{c(y)}-c_{x}(y) \frac{z_{e a}(y)-z(y)}{c(y)}\right) \cos \Lambda_{e a}
$$

where the value of $c_{m_{e a}}$ twists the wing nose-up about the local elastic axis node.

The wing spanwise derivatives $c_{l_{\delta_{n}}}, c_{y_{\delta_{n}}}$, and $c_{m_{e a} a_{n}}$ are calculated using a central difference method as in Eqs. 27, 28, and 29. Note at the subscript $\delta_{n}$ represents $\frac{\partial}{\partial \Delta \delta_{n}}$ where $\Delta \delta_{n}$ is a change in the deflection of the $n$-th camber segments of the VCCTEF from the nominal setting $\bar{\delta}$. For the first configuration of the VCCTEF $n=3$, while $n=2$ for the second configuration of the VCCTEF.

$$
\begin{gathered}
c_{l_{\delta_{n}}}=\frac{c_{l}(\bar{\delta}+\varepsilon)-c_{l}(\bar{\delta}-\varepsilon)}{2 \varepsilon} \\
c_{y_{\delta_{n}}}=\frac{c_{y}(\bar{\delta}+\varepsilon)-c_{y}(\bar{\delta}-\varepsilon)}{2 \varepsilon} \\
c_{m_{e a} \delta_{\delta_{n}}}=\frac{c_{m_{e a}}(\bar{\delta}+\varepsilon)-c_{m_{e a}}(\bar{\delta}-\varepsilon)}{2 \varepsilon}
\end{gathered}
$$

The wing sectional drag derivative coefficient is modeled using a second order fit, where the values $c_{d_{\delta_{n}}}$ and $c_{d_{\left(\delta_{n}\right)^{2}}}$ are determined as

$$
\begin{gathered}
c_{d_{\delta_{n}}}=\frac{c_{d}(\bar{\delta}+\varepsilon)-c_{d}(\bar{\delta}-\varepsilon)}{2 \varepsilon} \\
c_{d_{\left(\delta_{n}\right)^{2}}}=\frac{c_{d}(\bar{\delta}+\varepsilon)+c_{d}(\bar{\delta}-\varepsilon)-2 c_{d}(\bar{\delta})}{2 \varepsilon^{2}}
\end{gathered}
$$




\section{Aircraft Total Derivatives}

VORVIEW/VORLAX returns the total lift, drag, and pitching moment coefficients for an input aerodynamic configuration automatically. This corresponds to the aircraft coefficients, $C_{L}, C_{D}$, and $C_{m}$ where the pitching moment is given about the user input value for the aircraft center of gravity (c.g.). The aircraft rolling moment, yawing moment, and side force coefficients, $C_{l}, C_{n}$, and $C_{Y}$ respectively, can be calculated by integrating the spanwise distributions determined in Eqs. 21-23. The moment coefficients $C_{l}, C_{m}$ and $C_{n}$ utilize aircraft conventions where the moments are determined at the aircraft c.g. about the roll, pitch, and yaw axes respectively. Note that axes are not the same as the global axes defined in Fig. 8 .

$$
\begin{gathered}
C_{l}=-\frac{1}{S b} \int_{-\frac{b}{2}}^{\frac{b}{2}} y c_{z}(y) c(y) d y \\
C_{n}=\frac{1}{S b} \int_{-\frac{b}{2}}^{\frac{b}{2}} y c_{x}(y) c(y) d y \\
C_{Y}=\frac{1}{S} \int_{-\frac{b}{2}}^{\frac{b}{2}} c_{y}(y) c(y) d y
\end{gathered}
$$

The aircraft coefficient derivatives with respect to the VCCTEF deflections are calculated using finite-difference.

$$
\begin{gathered}
C_{L_{\delta_{n}}}=\frac{C_{L}(\bar{\delta}+\varepsilon)-C_{L}(\bar{\delta}-\varepsilon)}{2 \varepsilon} \\
C_{D_{\delta_{n}}}=\frac{C_{D}(\bar{\delta}+\varepsilon)-C_{D}(\bar{\delta}-\varepsilon)}{2 \varepsilon} \\
C_{D_{\left(\delta_{n}\right)^{2}}}=\frac{C_{D}(\bar{\delta}+\varepsilon)+C_{D}(\bar{\delta}-\varepsilon)-C_{D}(\bar{\delta})}{2 \varepsilon^{2}} \\
C_{Y_{\delta_{n}}}=\frac{C_{Y}(\bar{\delta}+\varepsilon)-C_{Y}(\bar{\delta}-\varepsilon)}{2 \varepsilon} \\
C_{l_{\delta_{n}}}=\frac{C_{l}(\bar{\delta}+\varepsilon)-C_{l}(\bar{\delta}-\varepsilon)}{2 \varepsilon} \\
C_{m_{\delta_{n}}}=\frac{C_{m}(\bar{\delta}+\varepsilon)-C_{m}(\bar{\delta}-\varepsilon)}{2 \varepsilon} \\
C_{n_{\delta_{n}}}=\frac{C_{n}(\bar{\delta}+\varepsilon)-C_{n}(\bar{\delta}-\varepsilon)}{2 \varepsilon}
\end{gathered}
$$

\section{B. Results}

Several sets of results are presented in this section, and each set includes the results for both VCCTEF configurations. The rigid wing results represent the control derivative analysis conducted assuming the wing does not experience any aeroelastic deformation, while the stiff and flexible represent wing structural models of a baseline typical conventional stiffness wing and a reduced flexibility model in torsion and bending, respectively. The value for $\varepsilon$ used for each of the studies was $\varepsilon=1^{\circ}$. For spanwise sectional derivatives, only the results for the right wing are shown and the left wing results are expected to be identical due to symmetry.

\section{Cruise}

The cruise sectional coefficient derivatives with respect to deflection of the fast actuated, aftmost (or $n$-th) camber segments for each of the VCCTEF sections are analyzed about the cruise $C_{L}=0.51$. The corresponding angle of attack and wing shapes for the rigid, stiff, and flexible wing configurations are shown in Table 1. 


\section{Rigid WING RESULTS}

Figures 13-16 represent the change in the spanwise coefficients with respect to perturbation of each flap section's aftmost camber segments.
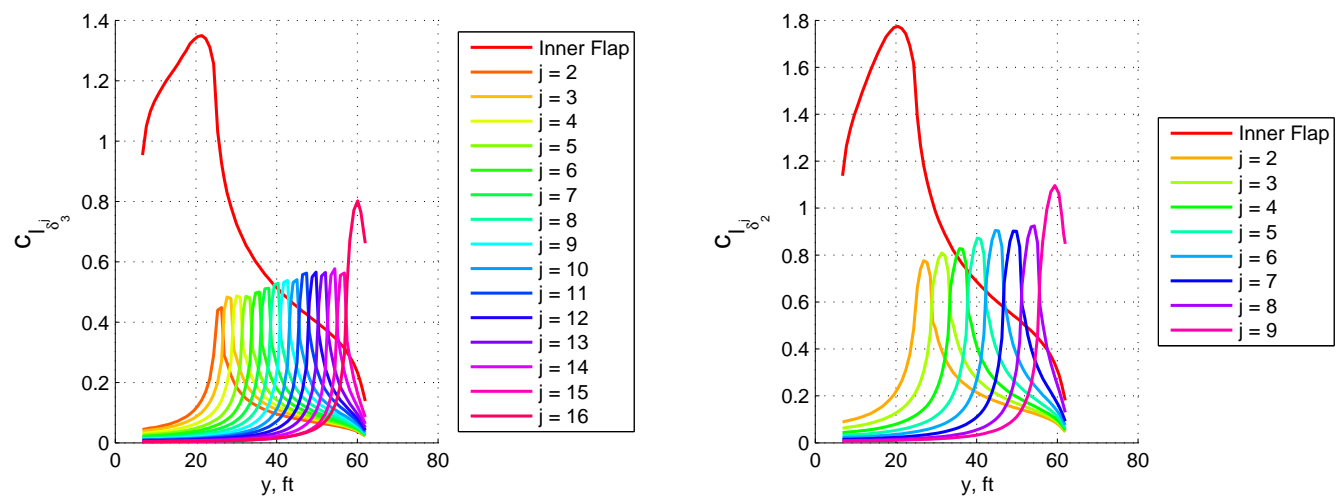

Figure 13. Rigid Wing Cruise Sectional Lift Spanwise Derivatives to VCCTEF, $c_{l_{\delta_{n}}}(y)$ (VCCTEF configuration \#1, left, VCCTEF configuration \#2, right)
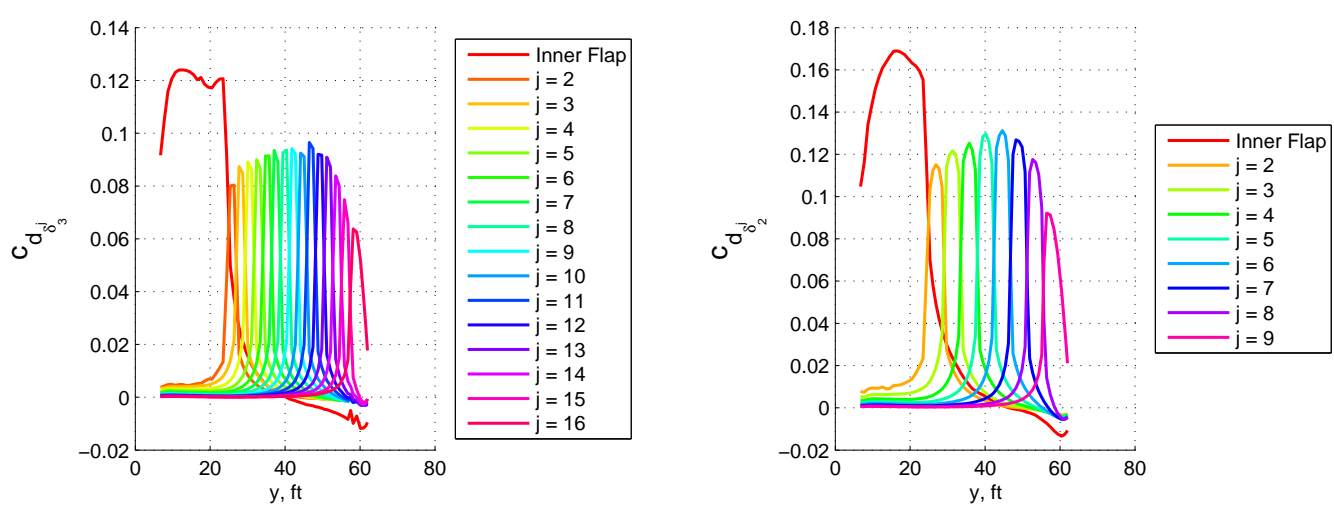

Figure 14. Rigid Wing Cruise Sectional Drag Spanwise Derivatives to VCCTEF, $c_{d_{\delta_{n}}}(y)$ (VCCTEF configuration \#1, left, VCCTEF configuration $\# 2$, right)
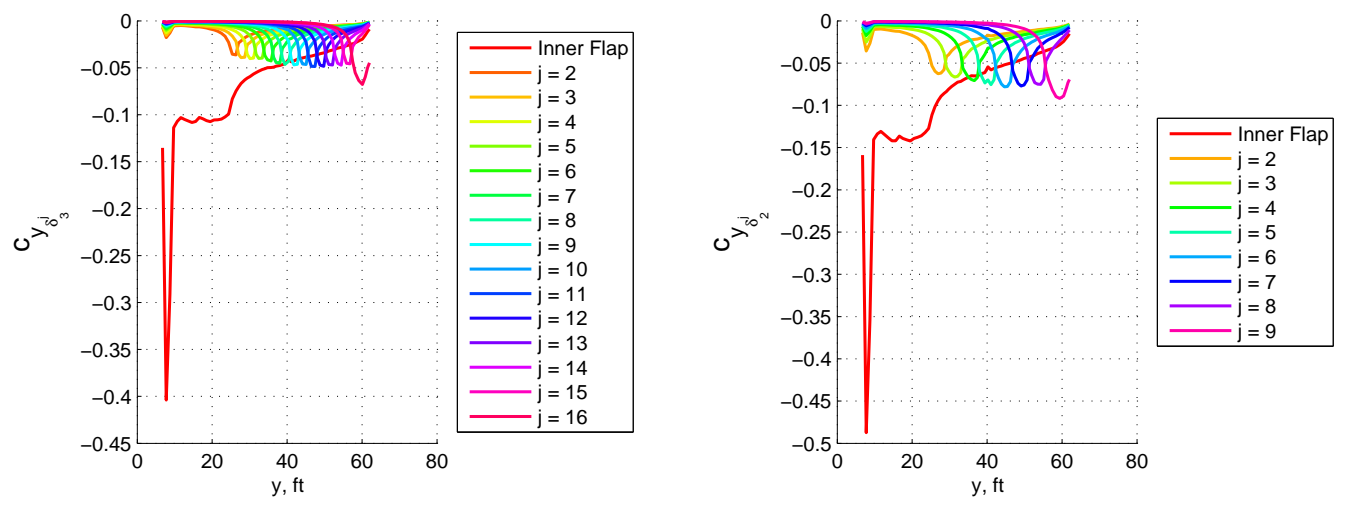

Figure 15. Rigid Wing Cruise Sectional Side Force Spanwise Derivatives to VCCTEF, $c_{y_{\delta_{n}}}(y)$ (VCCTEF configuration \#1, left, VCCTEF configuration \#2, right) 

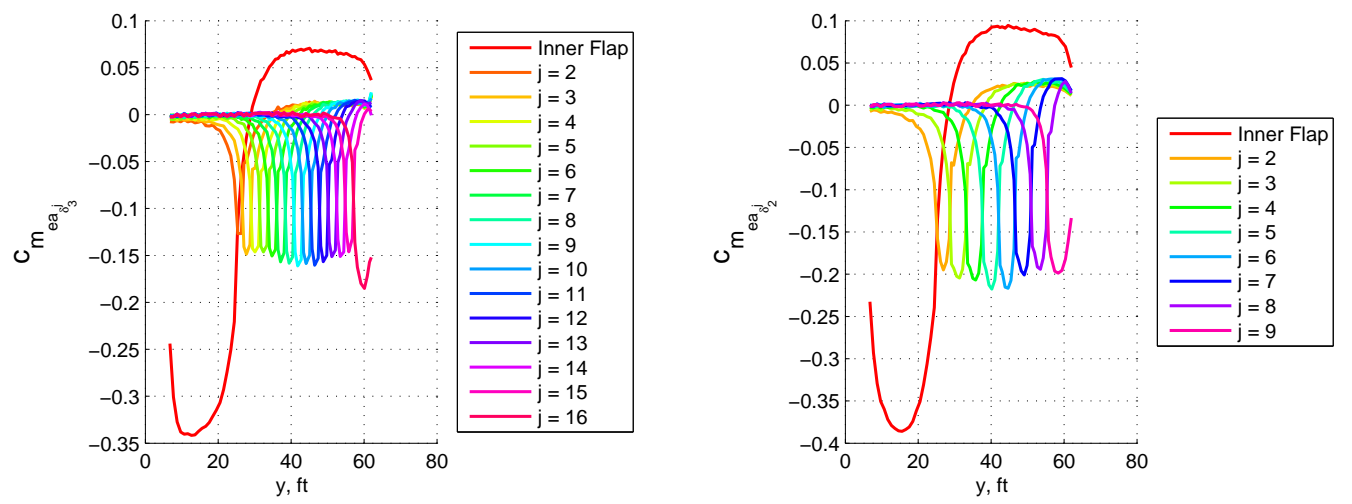

Figure 16. Rigid Wing Cruise Sectional Pitching Moment (about Elastic Axis) Spanwise Derivatives to VCCTEF, $c_{m_{e a_{\delta_{n}}}}(y)$ (VCCTEF configuration \#1, left, VCCTEF configuration \#2, right)

The total aircraft derivatives with respect to the VCCTEF for both VCCTEF configurations are shown in Figs. 17-22. The symmetric aircraft derivatives assume identical deflection of the VCCTEF on both the left and right wing, while the antisymmetric derivatives assume that positive deflection occurs on the left wing and negative deflection on the right.
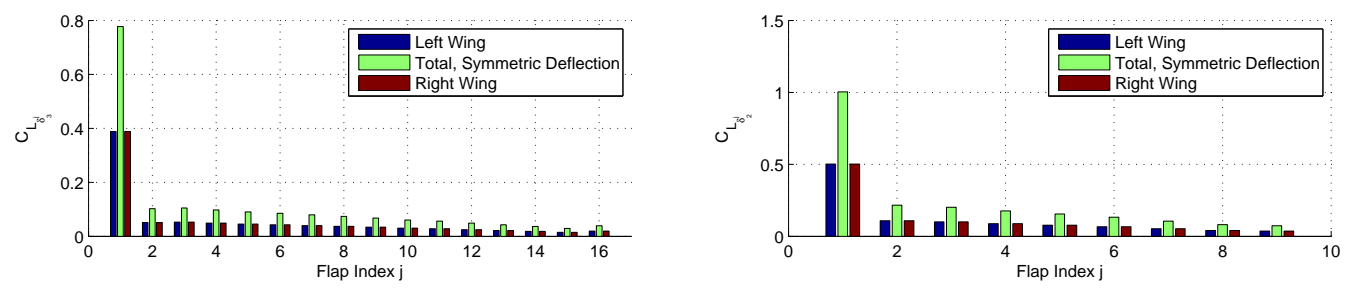

Figure 17. Rigid Wing Cruise Total Aircraft Lift Derivatives to VCCTEF, $C_{L_{\delta_{n}}}$ (VCCTEF configuration \#1, left, VCCTEF configuration \#2, right)
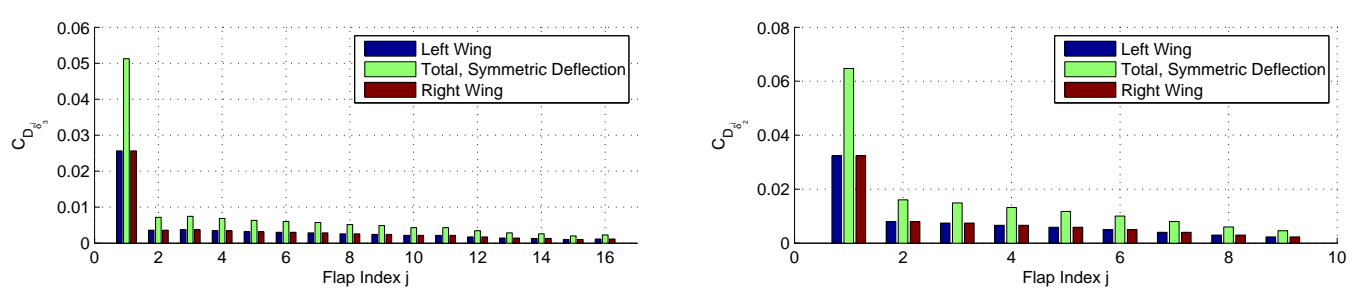

Figure 18. Rigid Wing Cruise Total Aircraft Drag Derivatives to VCCTEF, $C_{D_{\delta_{n}}}$ (VCCTEF configuration \#1, left, VCCTEF configuration \#2, right)
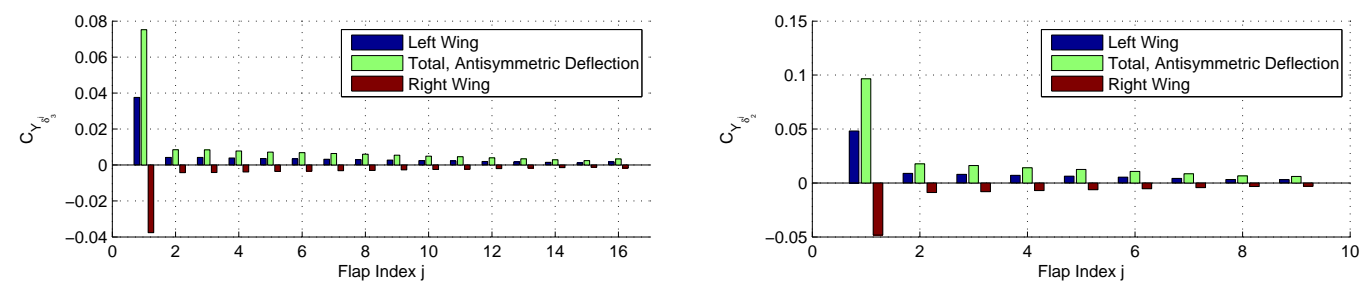

Figure 19. Rigid Wing Cruise Total Aircraft Side Force Derivatives to VCCTEF, $C_{Y_{\delta_{n}}}$ (VCCTEF configuration \#1, left, VCCTEF configuration \#2, right) 

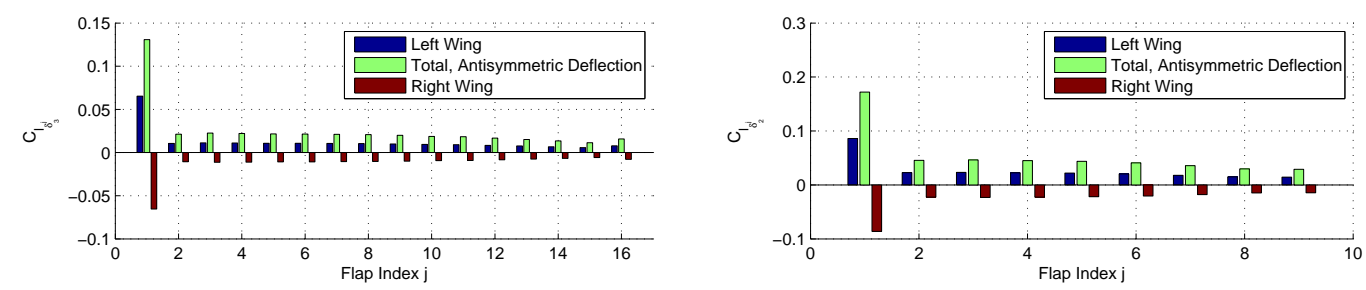

Figure 20. Rigid Wing Cruise Total Aircraft Rolling Moment Derivatives to VCCTEF, $C_{l_{\delta_{n}}}$ (VCCTEF configuration \#1, left, VCCTEF configuration \#2, right)
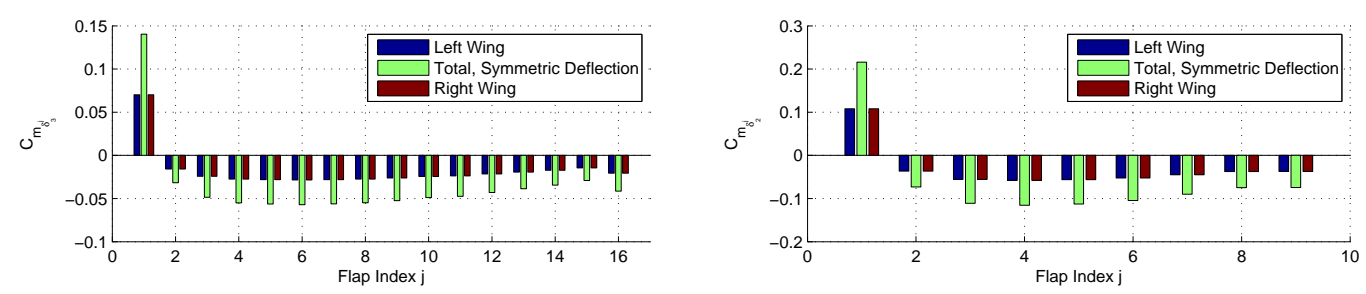

Figure 21. Rigid Wing Cruise Total Aircraft Pitching Moment Derivatives to VCCTEF, $C_{m_{\delta_{n}}}$ (VCCTEF configuration \#1, left, VCCTEF configuration \#2, right)
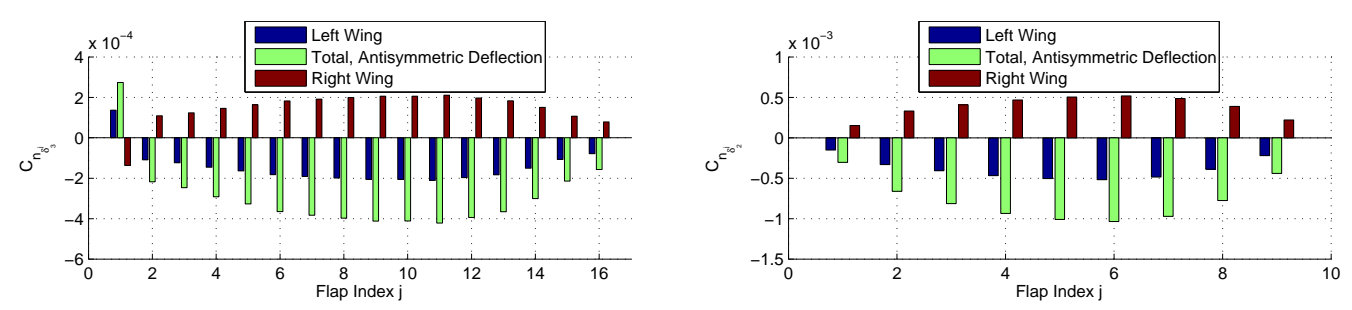

Figure 22. Rigid Wing Cruise Total Aircraft Yawing Moment Derivatives to VCCTEF, $C_{n_{\delta_{n}}}$ (VCCTEF configuration \#1, left, VCCTEF configuration \#2, right)

\section{StifF Wing Results}

Figures 23-26 are plots of the spanwise coefficient derivatives with respect to perturbation of each flap section's aftmost camber segments.
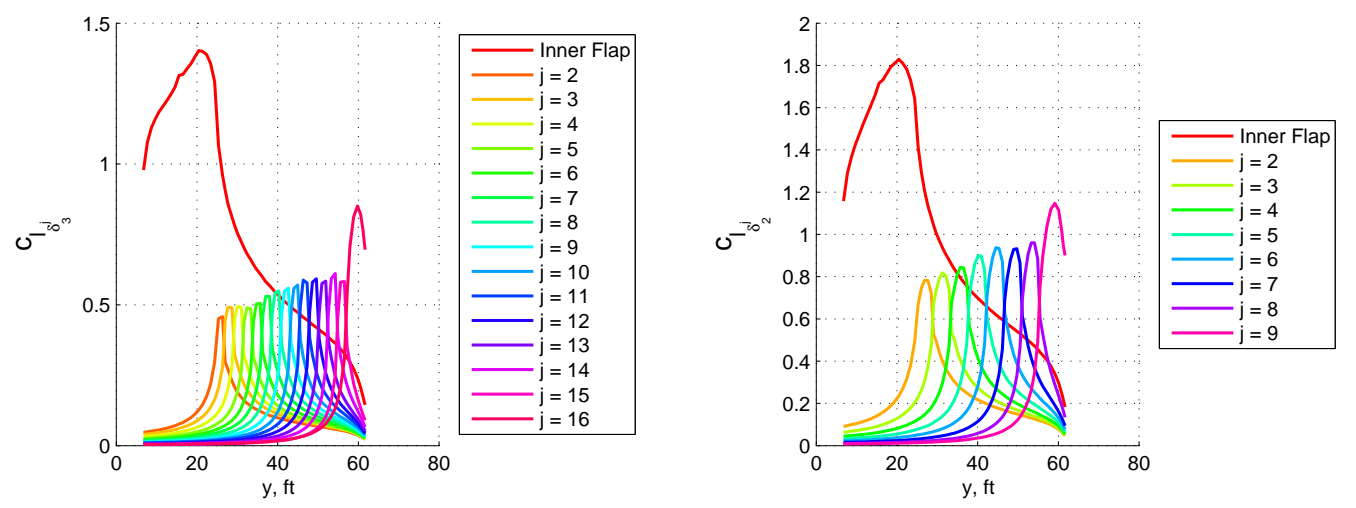

Figure 23. Stiff Wing Cruise Sectional Lift Spanwise Derivatives to VCCTEF, $c_{l_{\delta_{n}}}(y)$ (VCCTEF configuration \#1, left, VCCTEF configuration \#2, right) 

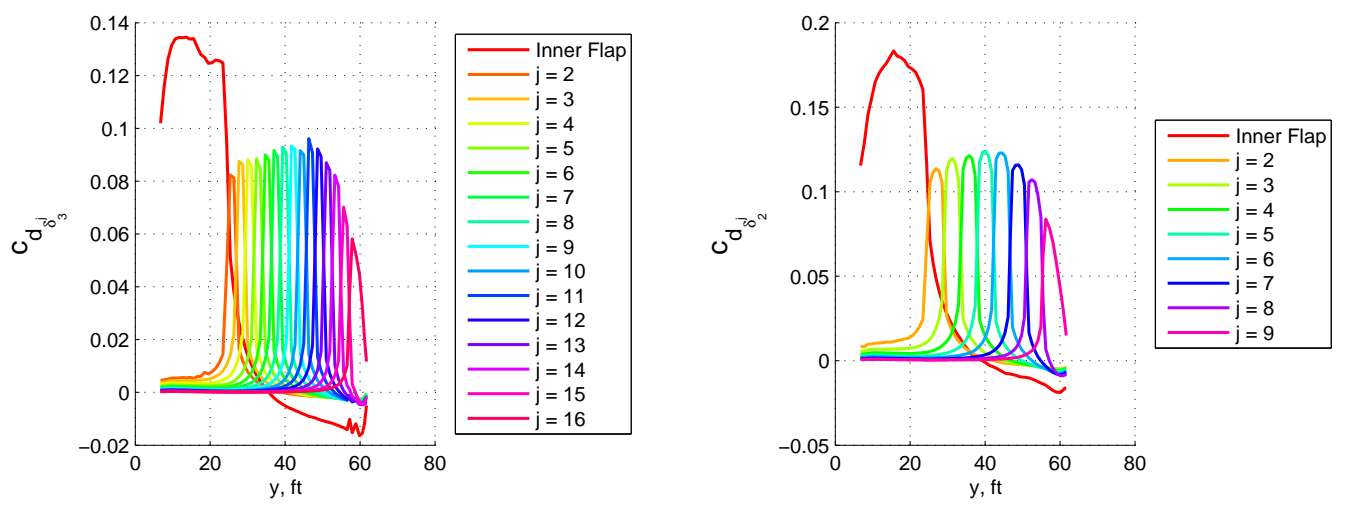

Figure 24. Stiff Wing Cruise Sectional Drag Spanwise Derivatives to VCCTEF, $c_{d_{\delta_{n}}}(y)$ (VCCTEF configuration \#1, left, VCCTEF configuration \#2, right)
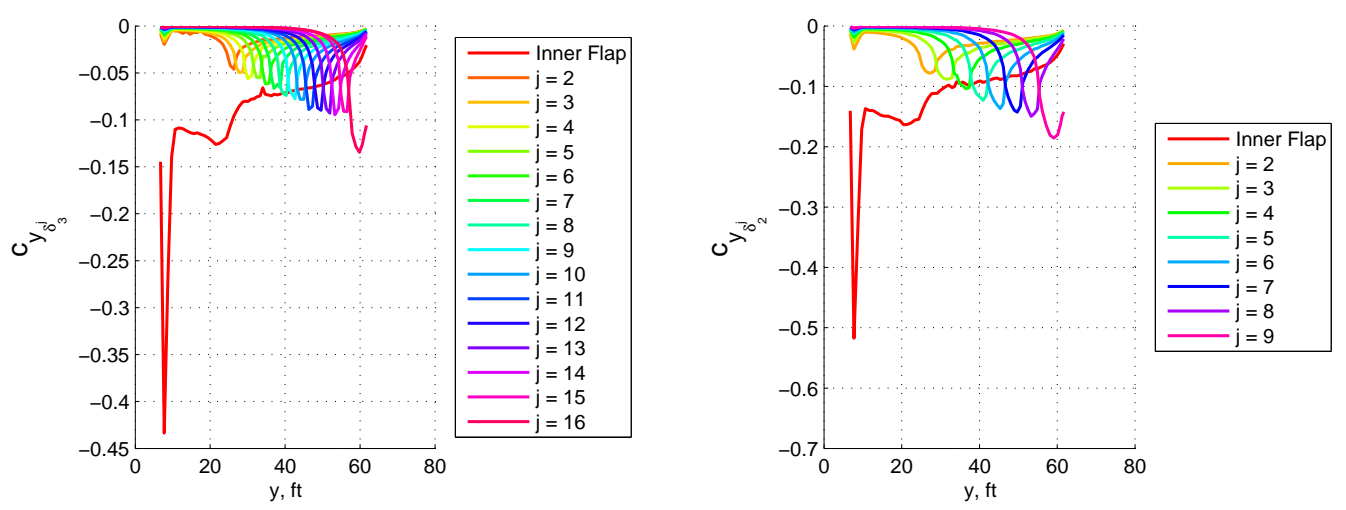

Figure 25. Stiff Wing Cruise Sectional Side Force Spanwise Derivatives to VCCTEF, $c_{y_{\delta_{n}}}(y)$ (VCCTEF configuration \#1, left, VCCTEF configuration \#2, right)
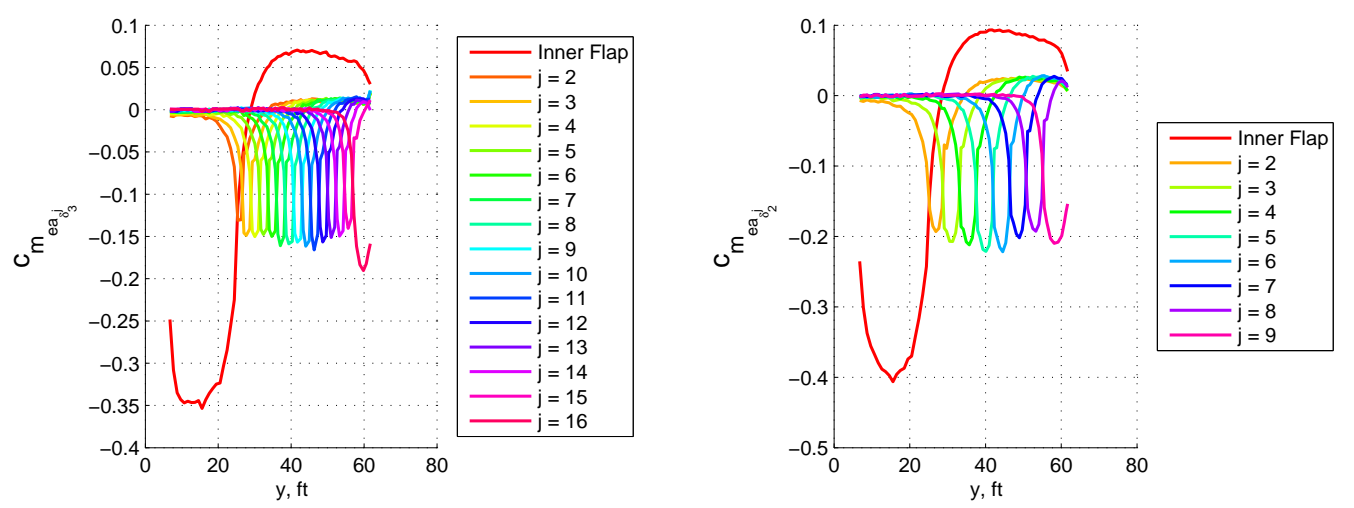

Figure 26. Stiff Wing Cruise Sectional Pitching Moment (about Elastic Axis) Spanwise Derivatives to VCCTEF, $c_{m_{e a} \delta_{n}}(y)$ (VCCTEF configuration \#1, left, VCCTEF configuration \#2, right)

The total aircraft derivatives with respect to the VCCTEF for the baseline stiff wing GTM are shown in Figs. 27-32. 

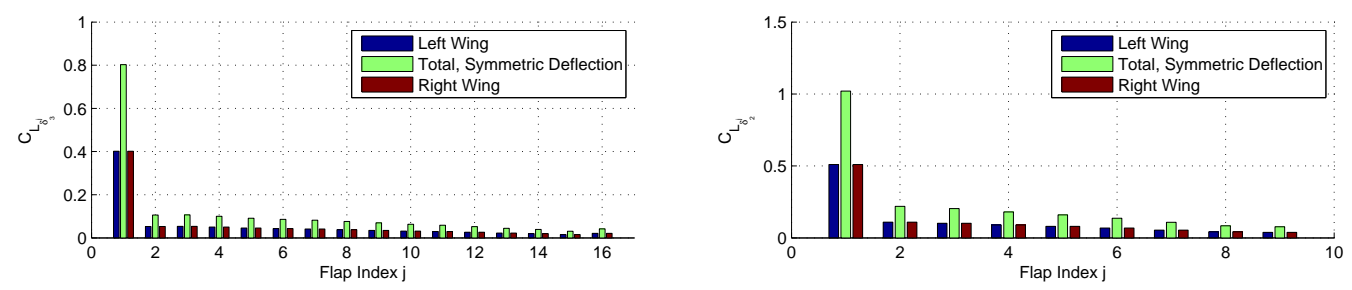

Figure 27. Stiff Wing Cruise Total Aircraft Lift Derivatives to VCCTEF, $C_{L_{\delta_{n}}}$ (VCCTEF configuration \#1, left, VCCTEF configuration \#2, right)
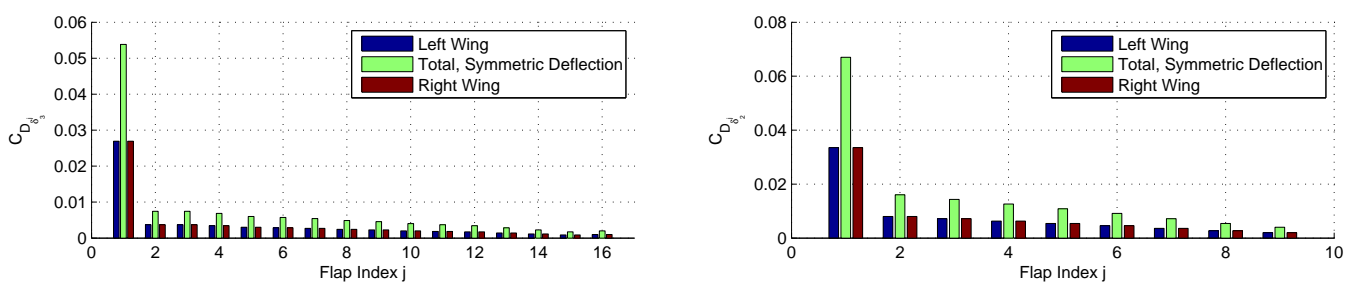

Figure 28. Stiff Wing Cruise Total Aircraft Drag Derivatives to VCCTEF, $C_{D_{\delta_{n}}}$ (VCCTEF configuration \#1, left, VCCTEF configuration \#2, right)
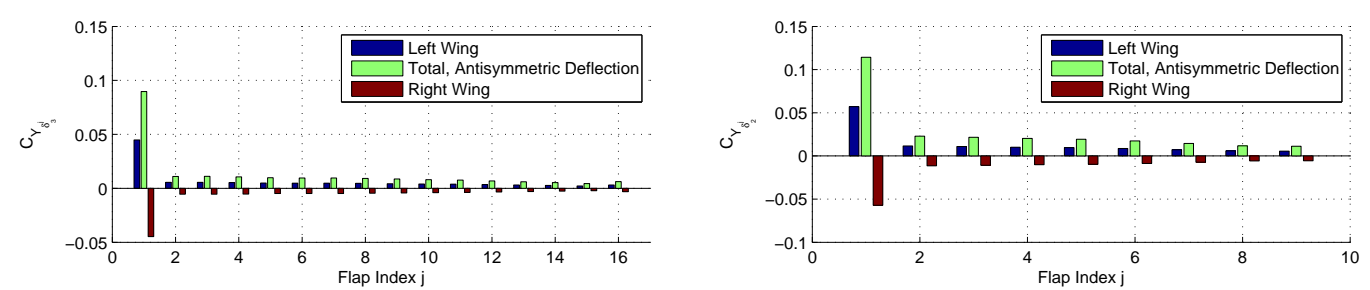

Figure 29. Stiff Wing Cruise Total Aircraft Side Force Derivatives to VCCTEF, $C_{Y_{\delta_{n}}}$ (VCCTEF configuration \#1, left, VCCTEF configuration \#2, right)
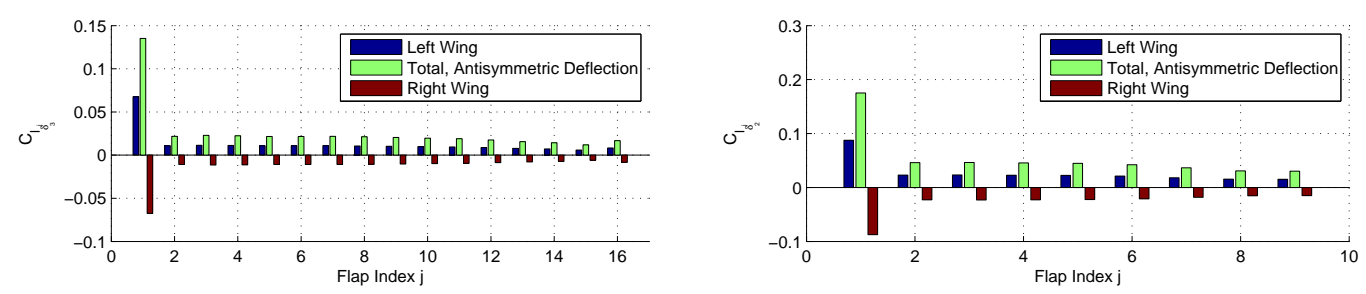

Figure 30. Stiff Wing Cruise Total Aircraft Rolling Moment Derivatives to VCCTEF, $C_{l_{\delta_{n}}}$ (VCCTEF configuration \#1, left, VCCTEF configuration \#2, right)
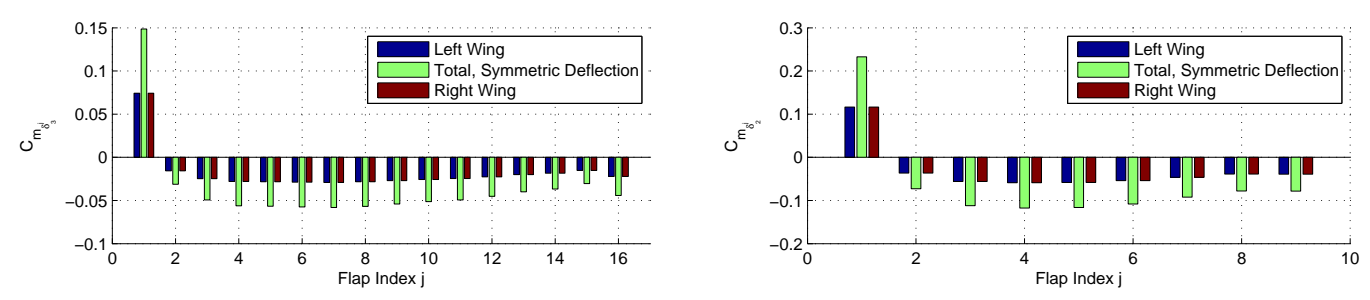

Figure 31. Stiff Wing Cruise Total Aircraft Pitching Moment Derivatives to VCCTEF, $C_{m_{\delta_{n}}}$ (VCCTEF configuration \#1, left, VCCTEF configuration \#2, right) 

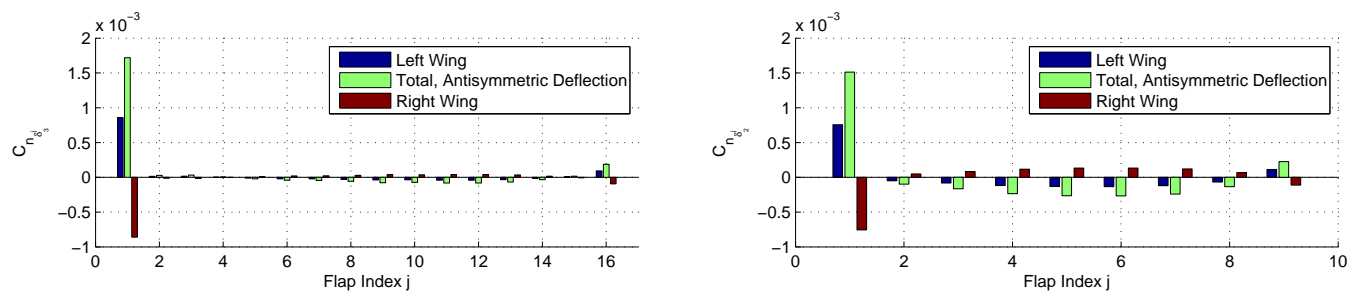

Figure 32. Stiff Wing Cruise Total Aircraft Yawing Moment Derivatives to VCCTEF, $C_{n_{\delta_{n}}}$ (VCCTEF configuration \#1, left, VCCTEF configuration \#2, right)

FLEXIBLE Wing RESUlTS

Figures 33-36 are the spanwise derivatives with respect to the deflection of the aftmost camber segments of the VCCTEF for the flexible wing model.
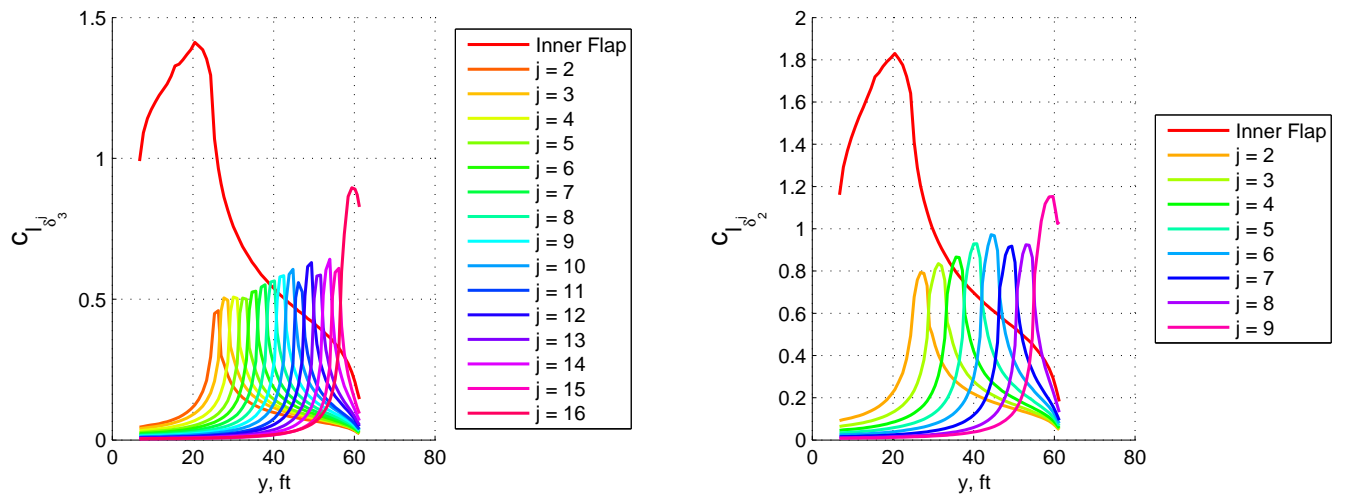

Figure 33. Flexible Wing Cruise Sectional Lift Spanwise Derivatives to VCCTEF, $c_{l_{\delta_{n}}}(y)$ (VCCTEF configuration \#1, left, VCCTEF configuration \#2, right)
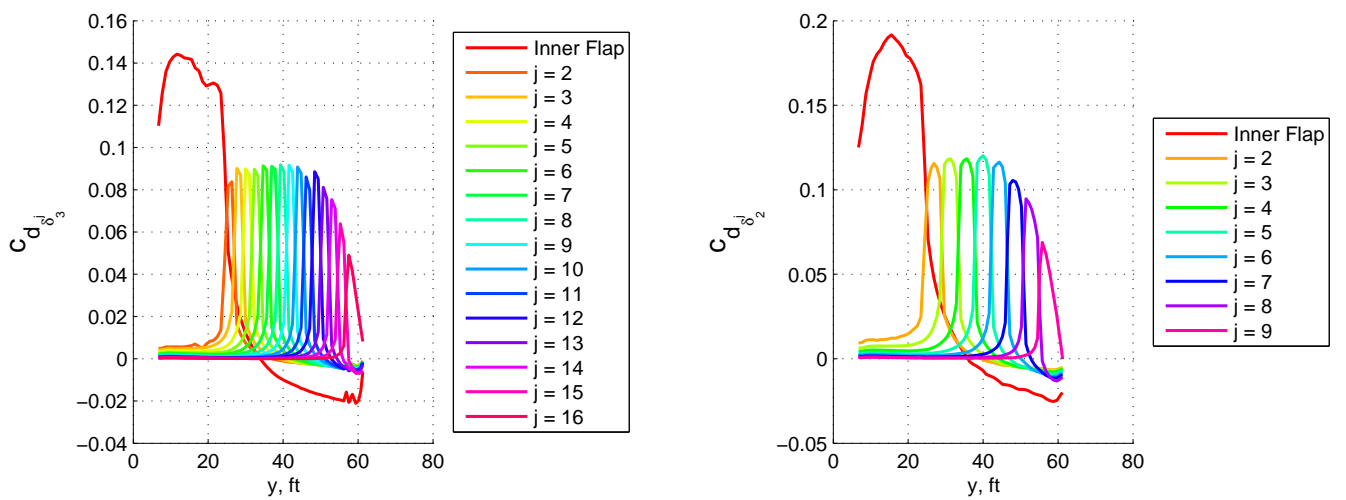

Figure 34. Flexible Wing Cruise Sectional Drag Spanwise Derivatives to VCCTEF, $c_{d_{\delta_{n}}}(y)$ (VCCTEF configuration \#1, left, VCCTEF configuration \#2, right) 

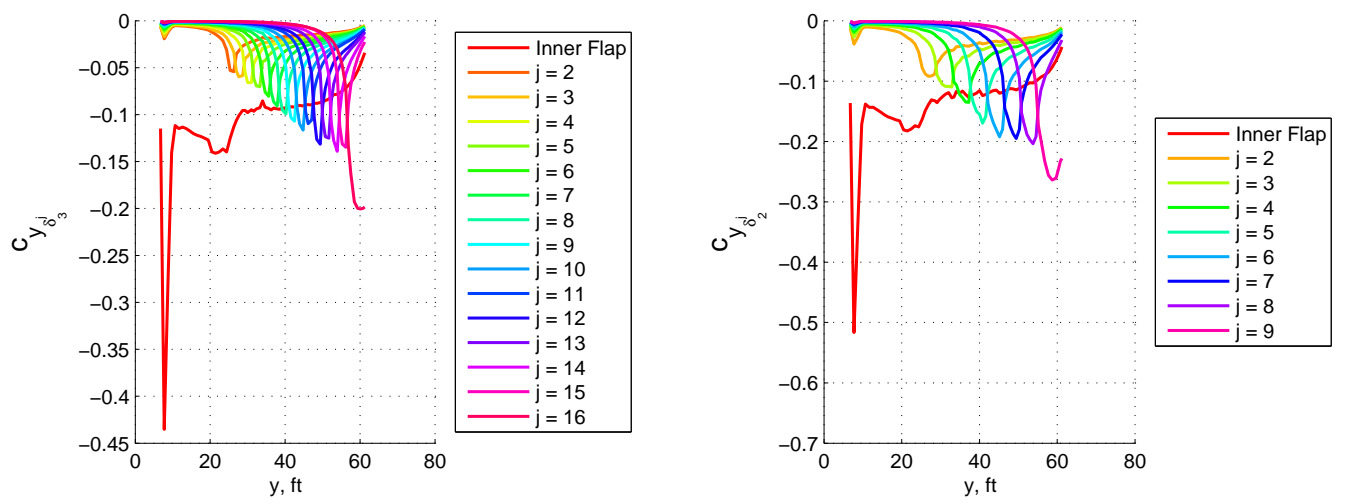

Figure 35. Flexible Wing Cruise Sectional Side Force Spanwise Derivatives to VCCTEF, $c_{y_{\delta_{n}}}(y)$ (VCCTEF configuration \#1, left, VCCTEF configuration \#2, right)
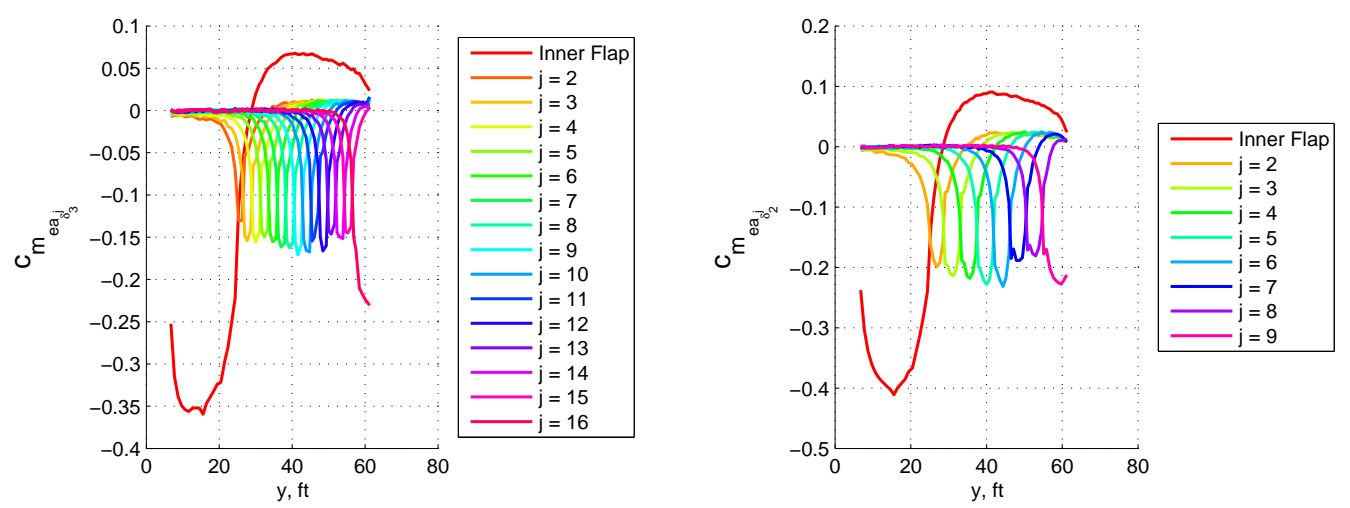

Figure 36. Flexible Wing Cruise Sectional Pitching Moment (about Elastic Axis) Spanwise Derivatives to VCCTEF, $c_{m_{e a_{\delta_{n}}}}(y)$ (VCCTEF configuration \#1, left, VCCTEF configuration \#2, right)

Figures 37-42 show the total aircraft derivatives with respect to the VCCTEF for the reduced stiffness flexible wing GTM.
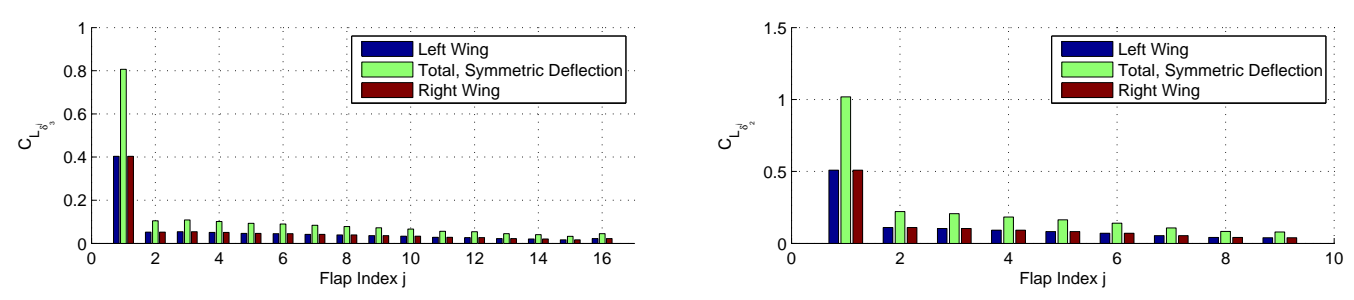

Figure 37. Flexible Wing Cruise Total Aircraft Lift Derivatives to VCCTEF, $C_{L_{\delta_{n}}}$ (VCCTEF configuration \#1, left, VCCTEF configuration \#2, right) 

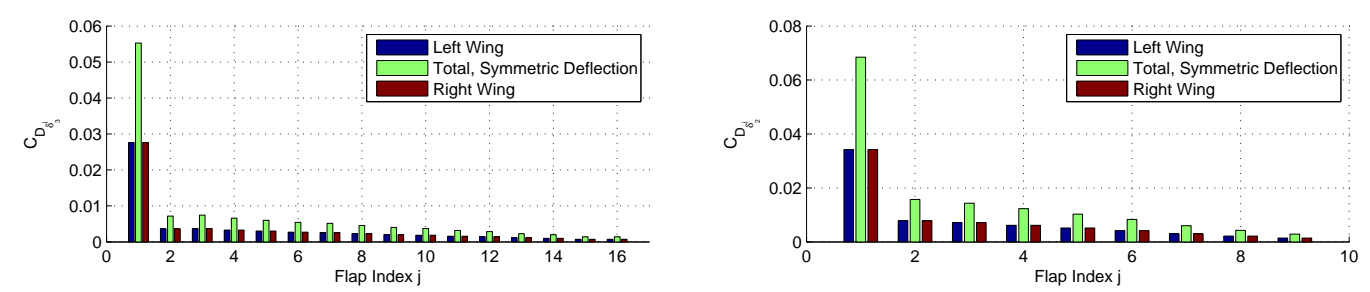

Figure 38. Flexible Wing Cruise Total Aircraft Drag Derivatives to VCCTEF, $C_{D_{\delta_{n}}}$ (VCCTEF configuration \#1, left, VCCTEF configuration \#2, right)
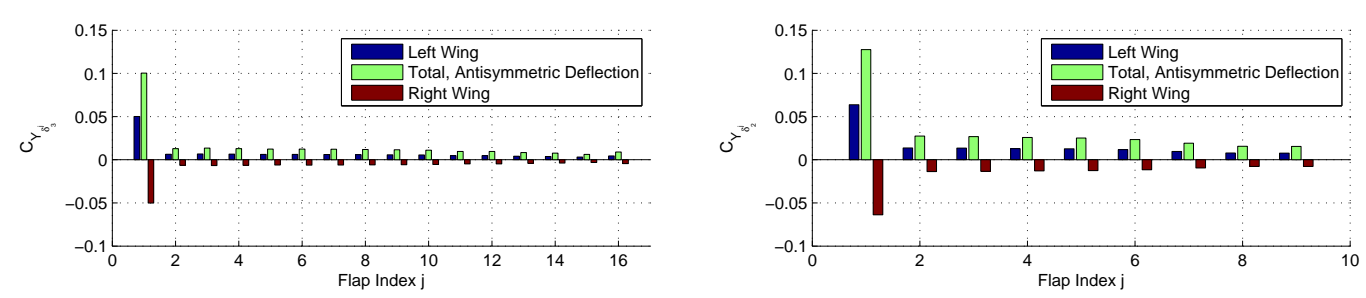

Figure 39. Flexible Wing Cruise Total Aircraft Side Force Derivatives to VCCTEF, $C_{Y_{\delta_{n}}}$ (VCCTEF configuration \#1, left, VCCTEF configuration \#2, right)
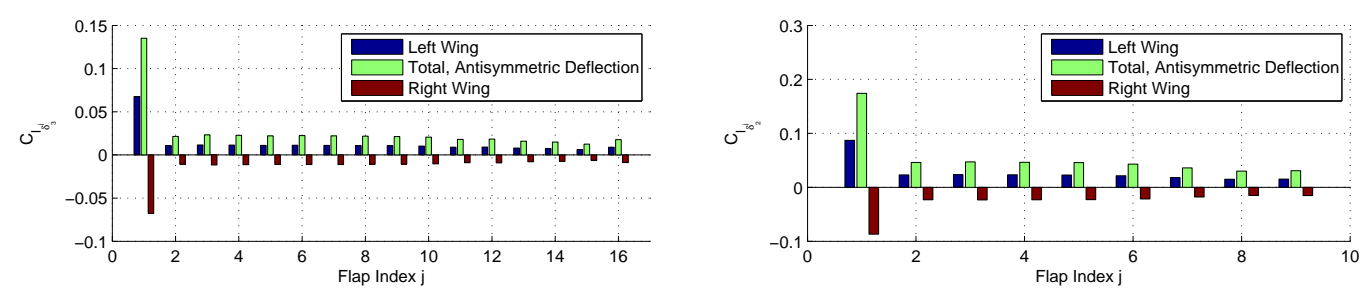

Figure 40. Flexible Wing Cruise Total Aircraft Rolling Moment Derivatives to VCCTEF, $C_{l_{\delta_{n}}}$ (VCCTEF configuration \#1, left, VCCTEF configuration \#2, right)
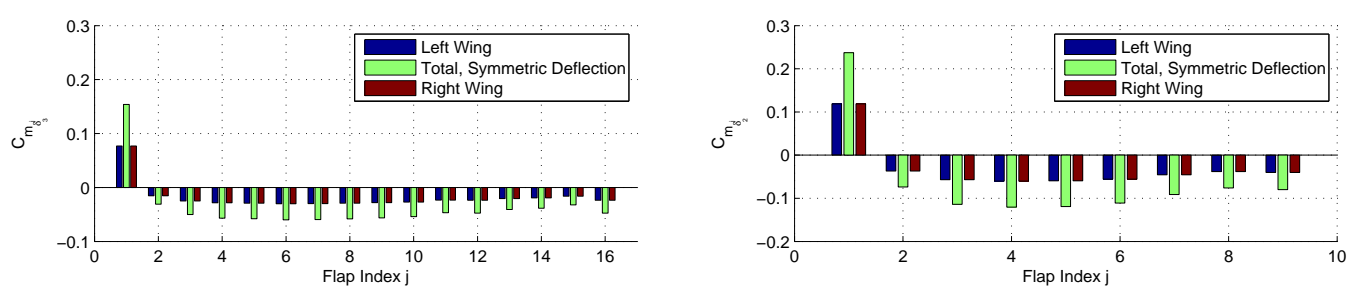

Figure 41. Flexible Wing Cruise Total Aircraft Pitching Moment Derivatives to VCCTEF, $C_{m_{\delta_{n}}}$ (VCCTEF configuration \#1, left, VCCTEF configuration \#2, right)
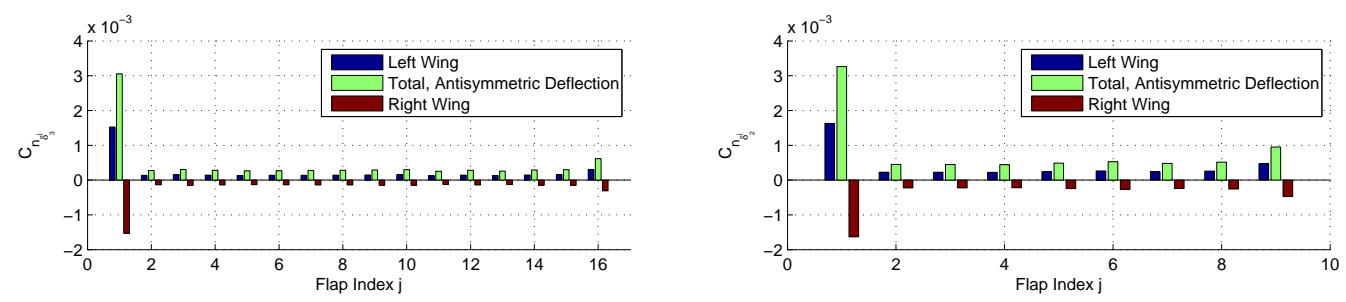

Figure 42. Flexible Wing Cruise Total Aircraft Yawing Moment Derivatives to VCCTEF, $C_{n_{\delta_{n}}}$ (VCCTEF configuration \#1, left, VCCTEF configuration \#2, right) 


\section{Cruise Fast Actuated VCCTEF Control Derivatives}

Let the subscript $\delta_{f}$ represent deflection of the aftmost camber segments (the $n$-th camber segments) of the outer flap sections of the VCCTEF (sections index 2 through $m$ ). That is, deflection of the fast actuated portion of the VCCTEF is $\delta_{f}=\sum_{j=2}^{m} \delta_{n}$. Also, let $\delta_{f, s}$ represent symmetric deflection of the fast actuated portion of the VCCTEF, and let $\delta_{f, a}$ represent asymmetric deflection of the fast actuated portion of the VCCTEF where positive deflection occurs on the left or port side wing. The total aircraft derivative for the fast actuated portion of the VCCTEF used for maneuvering are thus summarized in Table 3. For comparison, the control derivative values for the rigid wing GTM with conventional ailerons at $C_{L}=0.51$ (or corresponding $\alpha=3.791^{\circ}$ at $M=0.797$ ) are included for comparison.

\begin{tabular}{|c|c|c|c|c|c|c|c|}
\hline Aircraft Configuration & $C_{L_{\delta_{f, s}}}$ & $C_{D_{\delta_{f, s}}}$ & $C_{D_{\left(\delta_{f, s}\right)^{2}}}$ & $C_{Y_{\delta_{f, a}}}\left(C_{\delta_{\delta a}}\right)$ & $C_{l_{\delta_{f, a}}}\left(C_{l_{\delta a}}\right)$ & $C_{m_{\delta_{f, s}}}$ & $C_{n_{\delta_{f, a}}}\left(C_{n_{\delta a}}\right)$ \\
\hline $\begin{array}{c}\text { Rigid Wing } \\
\text { VCCTEF Configuration \#1 }\end{array}$ & 1.0156 & 0.0713 & 0.3774 & 0.0822 & 0.2804 & -0.6955 & -0.0049 \\
\hline $\begin{array}{c}\text { Rigid Wing } \\
\text { VCCTEF Configuration \#2 }\end{array}$ & 1.1413 & 0.0845 & 0.4431 & 0.0924 & 0.3159 & -0.7563 & -0.0066 \\
\hline $\begin{array}{c}\text { Stiff Wing } \\
\text { VCCTEF Configuration \#1 }\end{array}$ & 1.0479 & 0.0684 & -0.0493 & 0.1238 & 0.2884 & -0.7169 & -0.0003 \\
\hline $\begin{array}{c}\text { Stiff Wing } \\
\text { VCCTEF Configuration \#2 }\end{array}$ & 1.1691 & 0.0796 & -0.1314 & 0.1389 & 0.3226 & -0.7753 & -0.0012 \\
\hline $\begin{array}{c}\text { Flexible Wing } \\
\text { VCCTEF Configuration \#1 }\end{array}$ & 1.0726 & 0.0633 & -0.5417 & 0.1613 & 0.2948 & -0.7367 & 0.0045 \\
\hline $\begin{array}{c}\text { Flexible Wing } \\
\text { VCCTEF Configuration \#2 }\end{array}$ & 1.1846 & 0.0742 & -0.4433 & 0.1790 & 0.3249 & -0.7860 & 0.0043 \\
\hline $\begin{array}{c}\text { Rigid Wing } \\
\text { Conventional Ailerons }\end{array}$ & - & - & - & 0.0376 & 0.1624 & - & -0.0138 \\
\hline
\end{tabular}

Table 3. Control Derivative Values for VCCTEF Maneuvering Sections at Cruise

The results in Table 3 as well as the results shown in the figures for the three subcases of the cruise analysis demonstrate that the effect of the aeroelastic wing shape does affect the control derivative values of the VCCTEF. It is observed that, though quite small, the value of the yawing moment coefficient derivative with respect to the VCCTEF, $C_{n_{\delta}}$ changes sign. The lift coefficient derivative $C_{L_{\delta}}$, as well as the related rolling moment coefficient $C_{l_{\delta}}$, both increase as the wing flexibility increases. The roll control authority of the VCCTEF is also much higher than that of the conventional aileron system at cruise.

\section{Low-Speed Take-Off}

The low-speed take-off sectional coefficient derivatives with respect to deflection of the fast actuated, aftmost (or $n$-th) camber segments are determined for $C_{L}=1.56$ at sea-level for the aircraft. At the low-speed take-off condition, the VCCTEF has been retracted from its maximum deflection of $\delta=45^{\circ}$ to a value at which the maneuvering VCCTEF sections can now be utilized to conduct roll maneuvers. The nominal flap setting $\bar{\delta}$ that the flap has been retracted to once the aircraft has accelerated to the low-speed take-off speed of $M=0.228$ needs to be determined.

\section{Determining Nominal Flap Setting at Low-Speed TaKe-OfF}

The nominal flap setting $\bar{\delta}$ for the VCCTEF at the low-speed take-off flight condition is determined as the value at which the VCCTEF possesses about the same roll control authority as that of a conventional aileron system. At the low-speed take-off condition, it is assumed that the flaps are not operating at the maximum value of $\delta=45^{\circ}$, and antisymmetric deflections of the VCCTEF are enabled. Sweeps of control derivative analyses are conducted for various different values of $\bar{\delta}$, and the values for $C_{l_{\delta_{f, a}}}$ are compared against the value of $C_{l_{\delta a}}$ for the GTM's conventional ailerons. For simplification, this analysis is only run on the rigid wing GTM from a nominal deflection of $\bar{\delta}=5^{\circ}$ up to $\bar{\delta}=40^{\circ}$. 

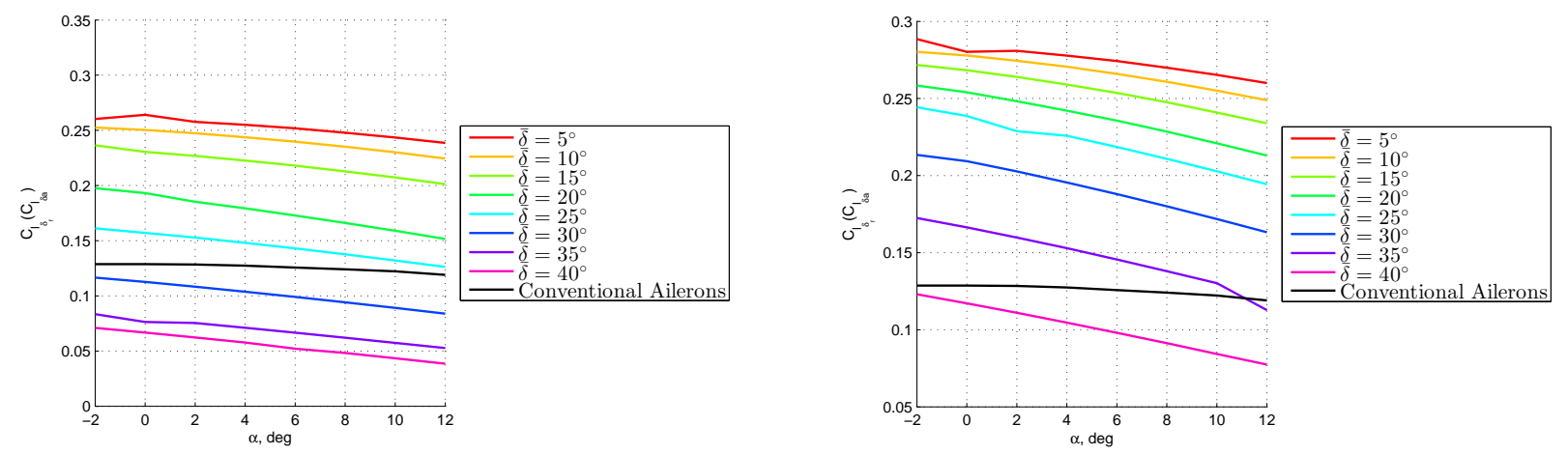

Figure 43. Rigid Wing VCCTEF Roll Control Derivatives $C_{l_{\delta_{f}}}$ (VCCTEF configuration \#1, left, VCCTEF configuration \#2, right)

Based on the results shown in Fig. 43, VCCTEF configuration \#1 should have an estimated nominal setting of at maximum $\bar{\delta}=25^{\circ}$ in order to maintain the same control authority as a conventional aileron system at the low-speed take-off flight condition for all angles of attack $\alpha=-2^{\circ}$ to $\alpha=10^{\circ}$. VCCTEF configuration \#2 is able to have a higher estimated nominal setting of $\bar{\delta}=35^{\circ}$. To ensure that incorporating wing flexibility into the wing system does not introduce roll reversal effects, the control derivatives for the VCCTEF configurations for rigid, stiff, and flexible wing models are evaluated for the low-speed take-off $C_{L}=1.56$. These are compared to the largest value of $C_{l_{\delta a}}$ for the conventional ailerons of $C_{l_{\delta a}}=0.1287$.
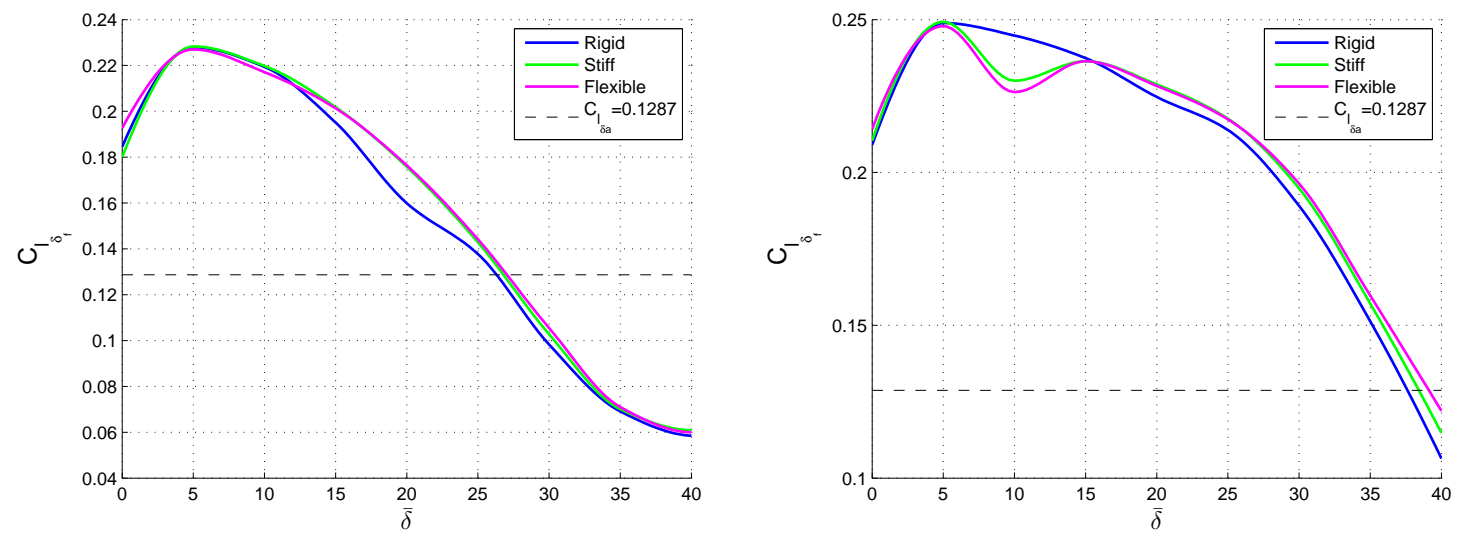

Figure 44. VCCTEF Roll Control Derivatives $C_{l_{\delta_{f}}}$ at Low-Speed Take-Off, $C_{L}=1.56$ (VCCTEF configuration \#1, left, VCCTEF configuration \#2, right)

The results in Fig. 44 demonstrate that the flexibility effects do not require a lower nominal setting than $\bar{\delta}=25^{\circ}$ for VCCTEF configuration $\# 1$, and $\bar{\delta}=35^{\circ}$ for VCCTEF configuration $\# 2$.

\section{RIGID WING RESULTS}

Figures 45-48 represent the change in the spanwise coefficients with respect to perturbation of each flap section's aftmost camber segments, about the nominal flap deflection $\bar{\delta}$ for low-speed take-off. 

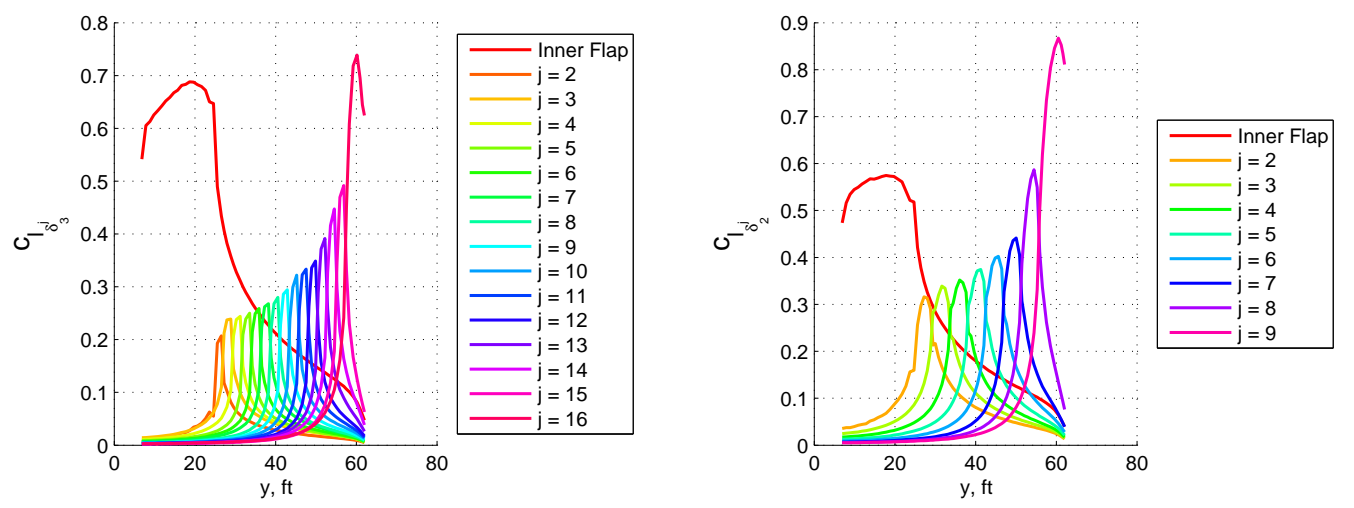

Figure 45. Rigid Wing Low-Speed Take-Off Sectional Lift Spanwise Derivatives to VCCTEF, $c_{l_{\delta_{n}}}(y)$ (VCCTEF configuration \#1, left, VCCTEF configuration \#2, right)
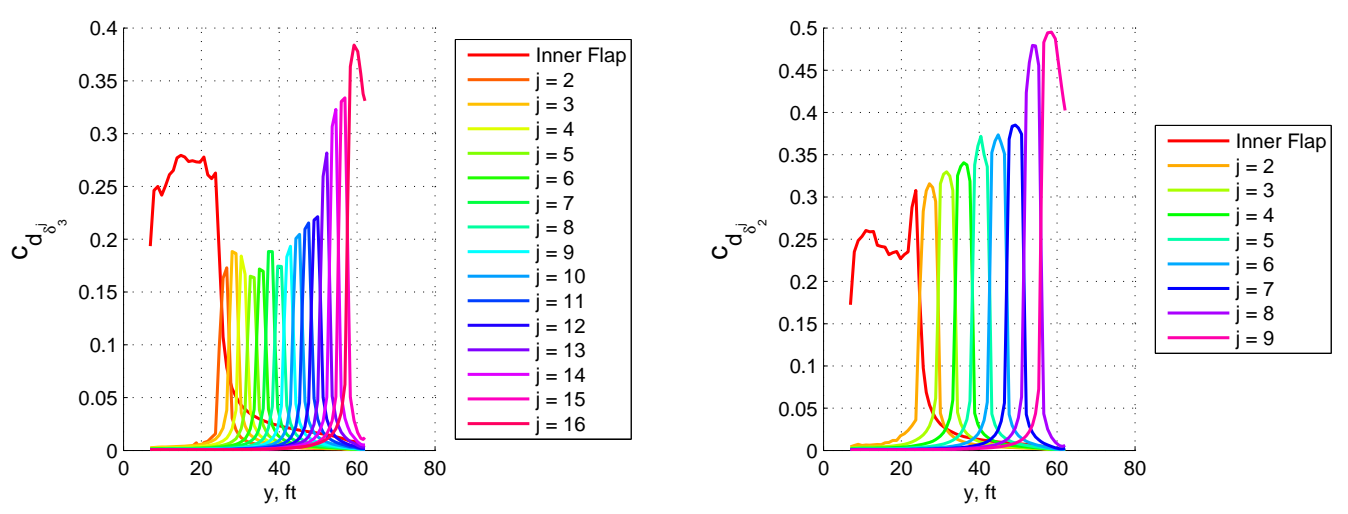

Figure 46. Rigid Wing Low-Speed Take-Off Sectional Drag Spanwise Derivatives to VCCTEF, $c_{d_{\delta_{n}}}(y)$ (VCCTEF configuration \#1, left, VCCTEF configuration \#2, right)
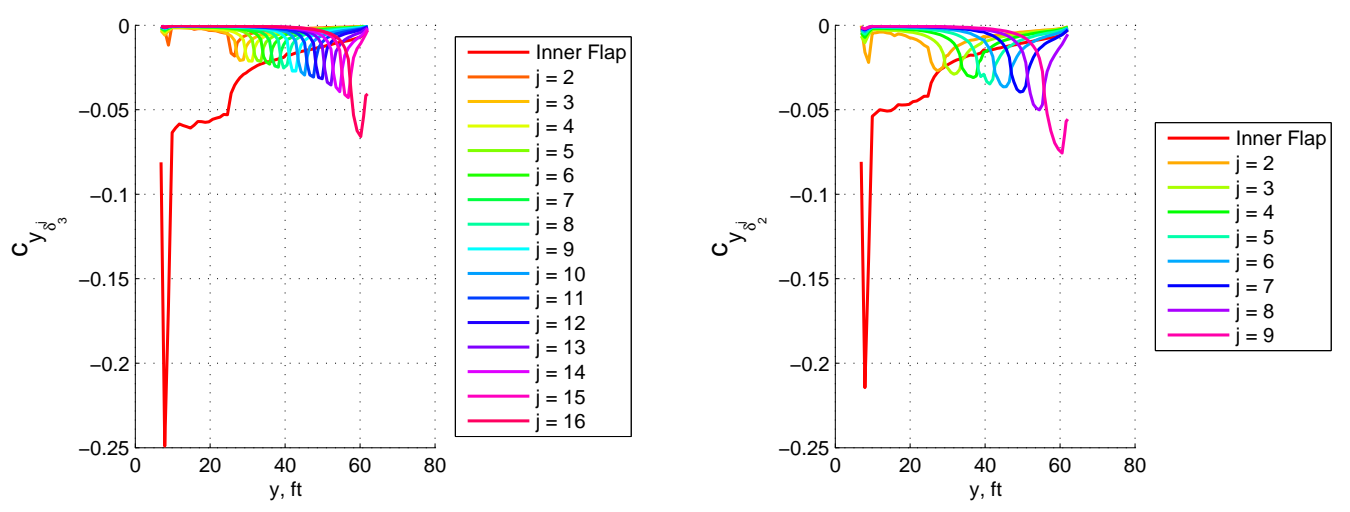

Figure 47. Rigid Wing Low-Speed Take-Off Sectional Side Force Spanwise Derivatives to VCCTEF, $c_{y_{\delta_{n}}}(y)$ (VCCTEF configuration \#1, left, VCCTEF configuration \#2, right) 

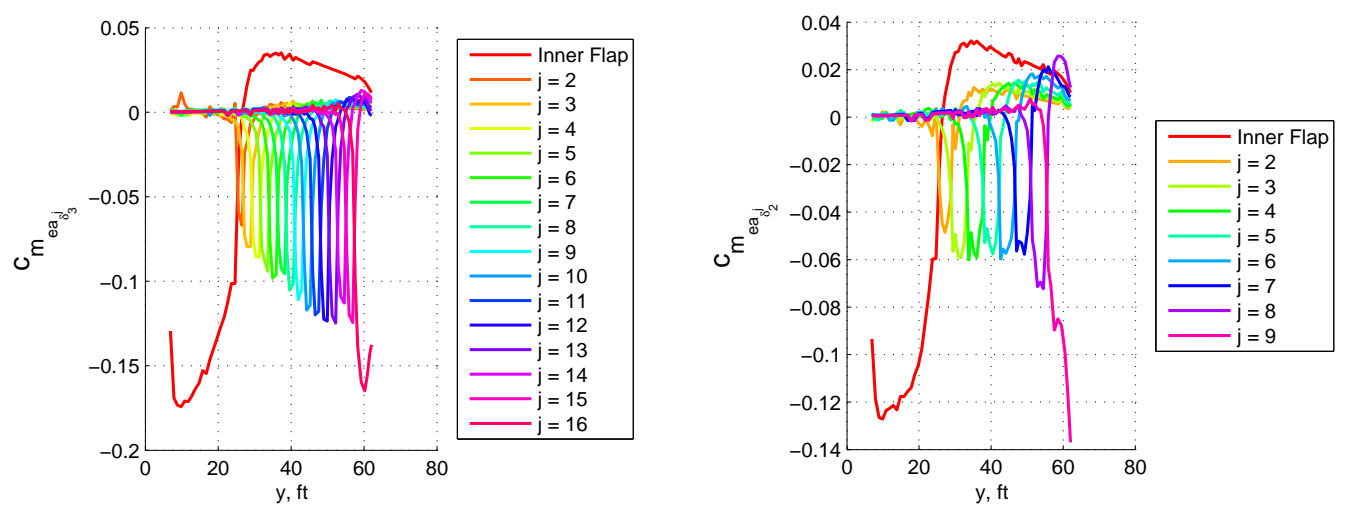

Figure 48. Rigid Wing Low-Speed Take-Off Sectional Pitching Moment (about Elastic Axis) Spanwise Derivatives to VCCTEF, $c_{m_{e} a_{\delta_{n}}}(y)$ (VCCTEF configuration \#1, left, VCCTEF configuration \#2, right)

The total aircraft derivatives with respect to the VCCTEF for both VCCTEF configurations are shown in Figs. 49-54.
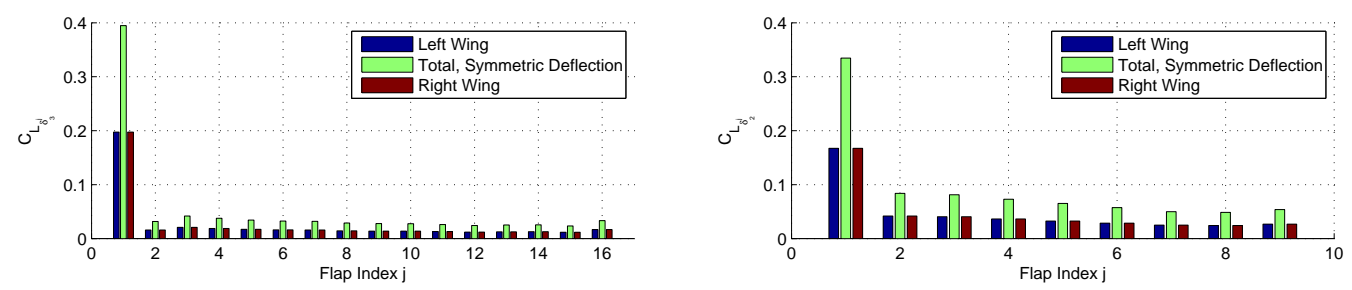

Figure 49. Rigid Wing Low-Speed Take-Off Total Aircraft Lift Derivatives to VCCTEF, $C_{L_{\delta_{n}}}$ (VCCTEF configuration \#1, left, VCCTEF configuration \#2, right)
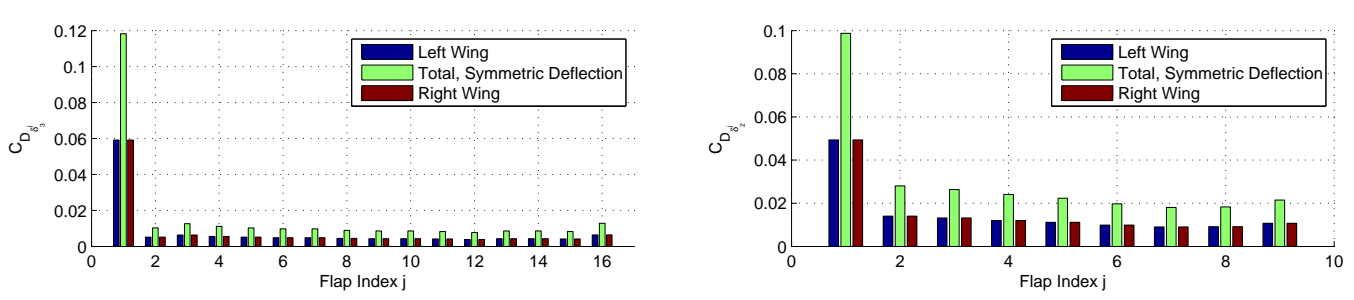

Figure 50. Rigid Wing Low-Speed Take-Off Total Aircraft Drag Derivatives to VCCTEF, $C_{D_{\delta_{n}}}$ (VCCTEF configuration \#1, left, VCCTEF configuration \#2, right)
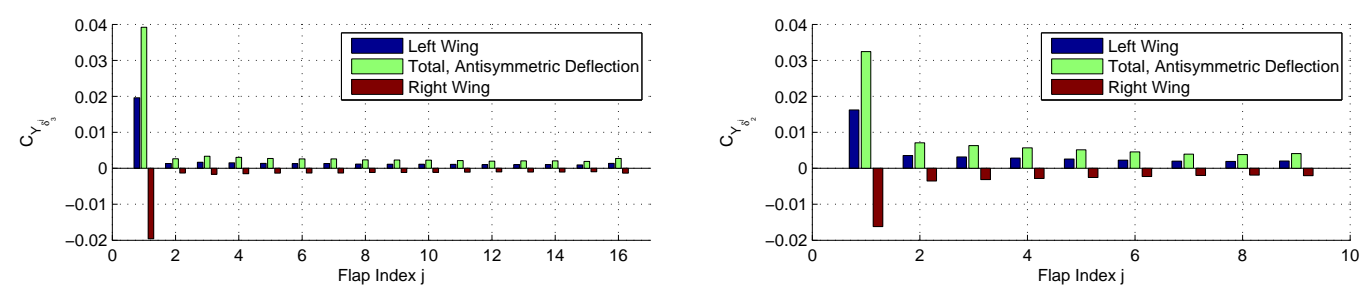

Figure 51. Rigid Wing Low-Speed Take-Off Total Aircraft Side Force Derivatives to VCCTEF, $C_{Y_{\delta_{n}}}$ (VCCTEF configuration \#1, left, VCCTEF configuration \#2, right) 

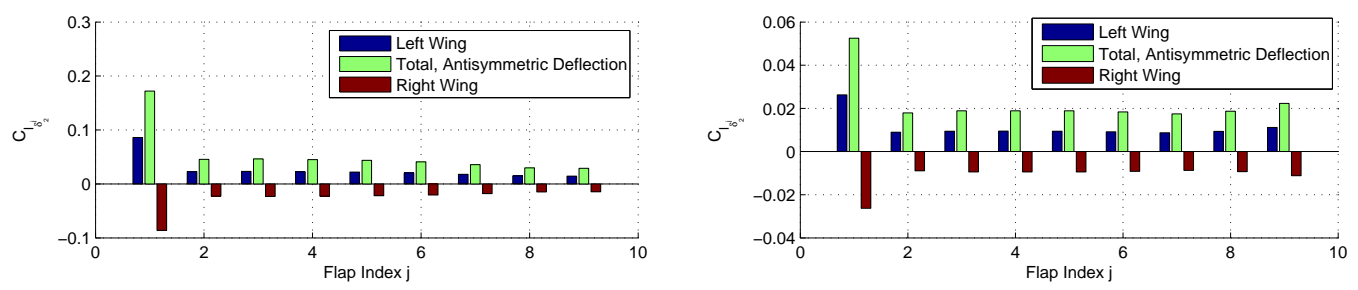

Figure 52. Rigid Wing Low-Speed Take-Off Total Aircraft Rolling Moment Derivatives to VCCTEF, $C_{l_{\delta_{n}}}$ (VCCTEF configuration \#1, left, VCCTEF configuration \#2, right)
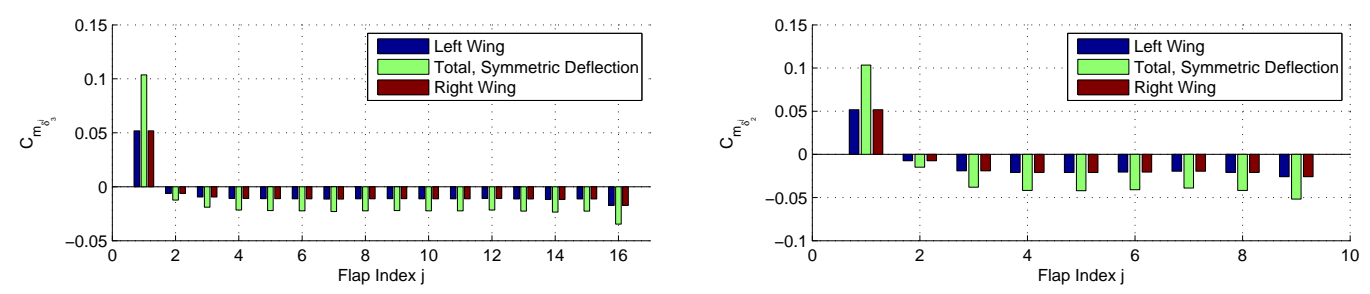

Figure 53. Rigid Wing Low-Speed Take-Off Total Aircraft Pitching Moment Derivatives to VCCTEF, $C_{m_{\delta_{n}}}$ (VCCTEF configuration \#1, left, VCCTEF configuration \#2, right)
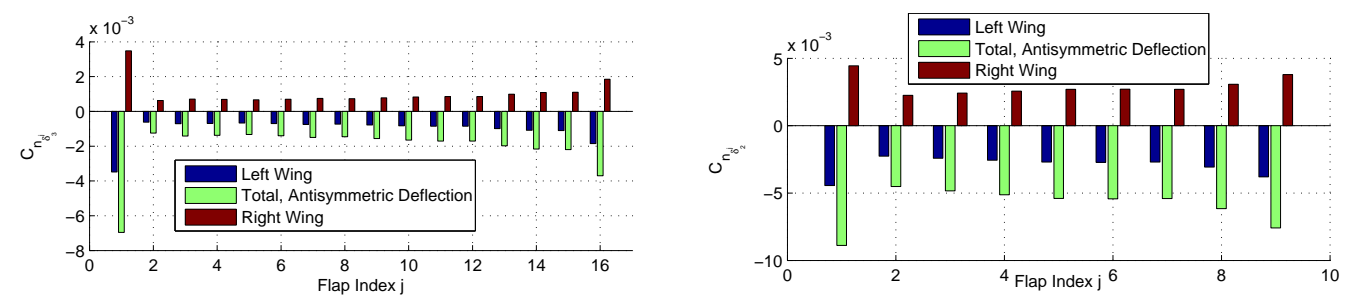

Figure 54. Rigid Wing Low-Speed Take-Off Total Aircraft Yawing Moment Derivatives to VCCTEF, $C_{n_{\delta_{n}}}$ (VCCTEF configuration \#1, left, VCCTEF configuration \#2, right)

\section{STIFF WING RESULTS}

Figures 55-58 are plots of the spanwise coefficients derivatives with respect to perturbation of each flap section's aftmost camber segments.
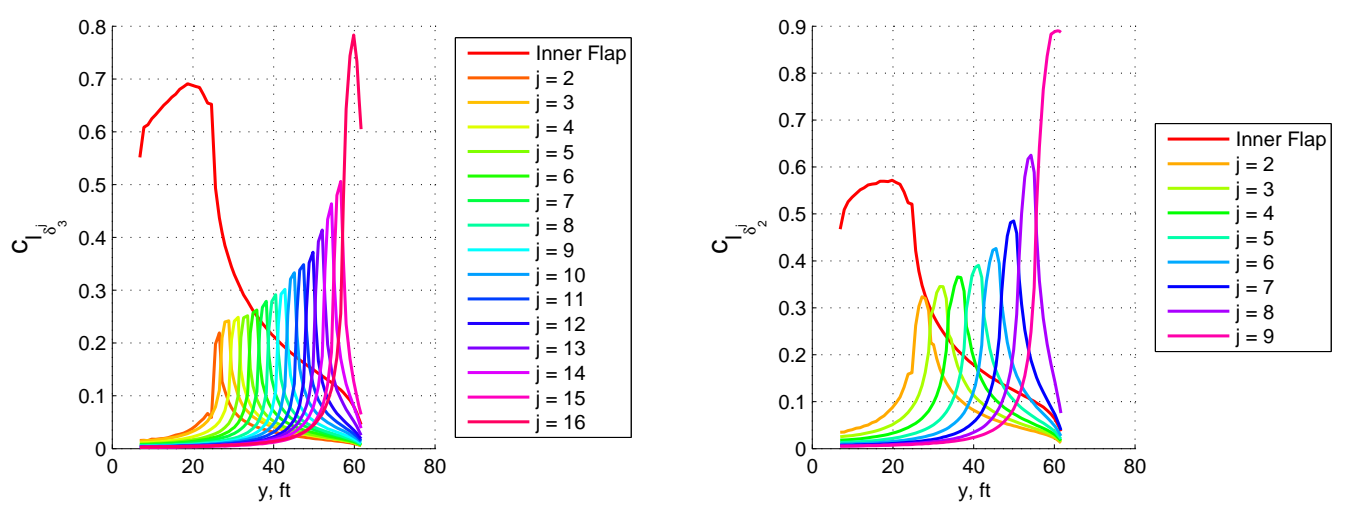

Figure 55. Stiff Wing Low-Speed Take-Off Sectional Lift Spanwise Derivatives to VCCTEF, $c_{l_{\delta_{n}}}(y)$ (VCCTEF configuration \#1, left, VCCTEF configuration \#2, right) 

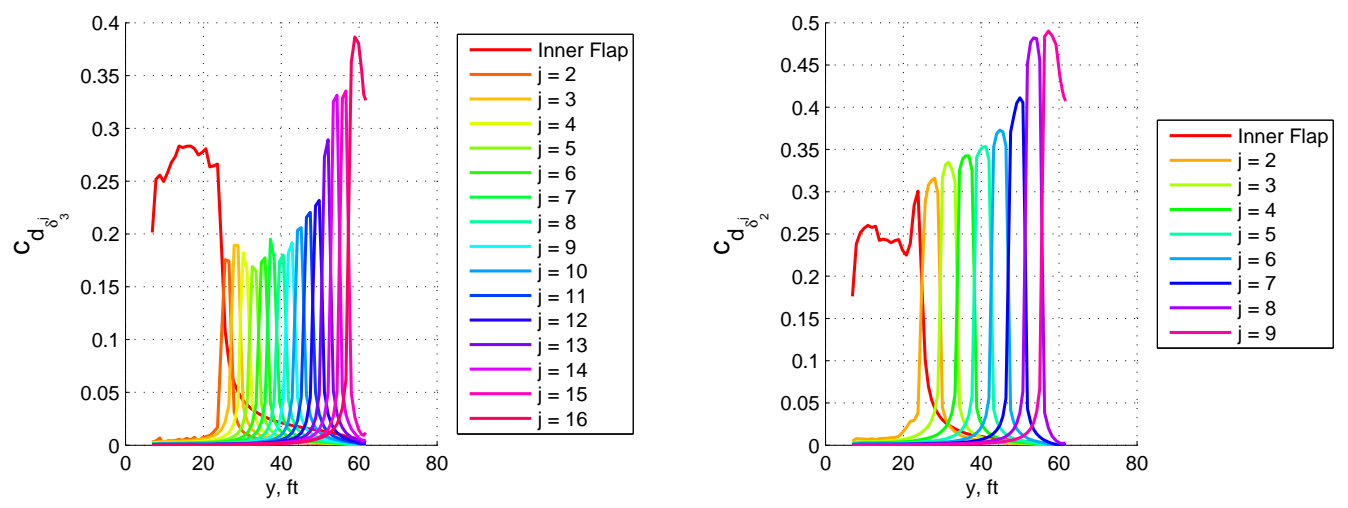

Figure 56. Stiff Wing Low-Speed Take-Off Sectional Drag Spanwise Derivatives to VCCTEF, $c_{d_{\delta_{n}}}(y)$ (VCCTEF configuration \#1, left, VCCTEF configuration \#2, right)
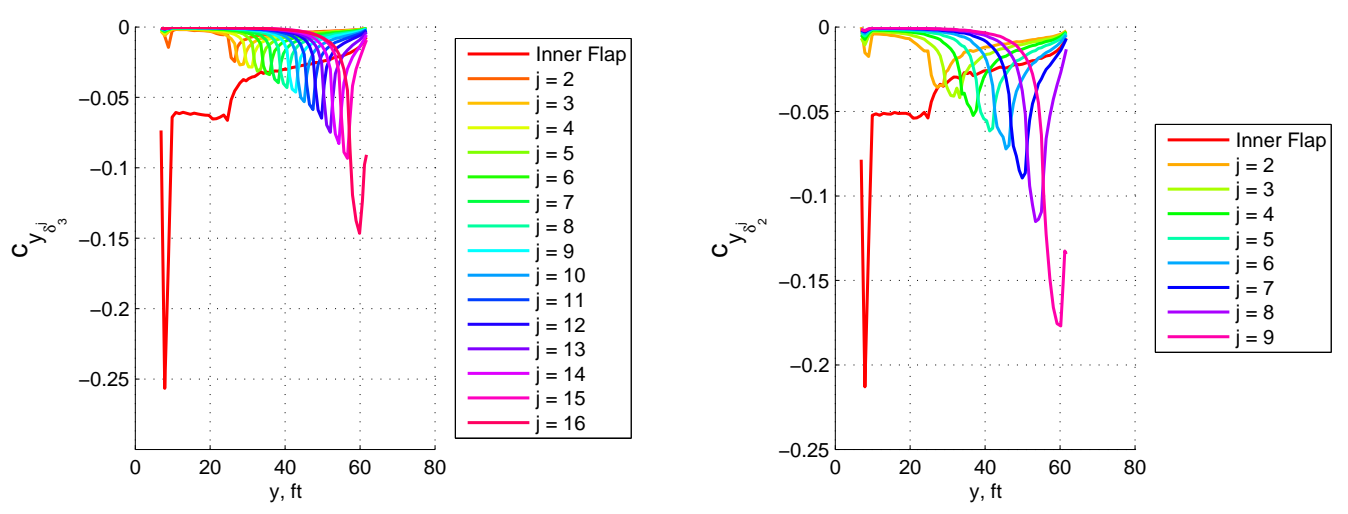

Figure 57. Stiff Wing Low-Speed Take-Off Sectional Side Force Spanwise Derivatives to VCCTEF, $c_{y_{\delta_{n}}}(y)$ (VCCTEF configuration \#1, left, VCCTEF configuration \#2, right)
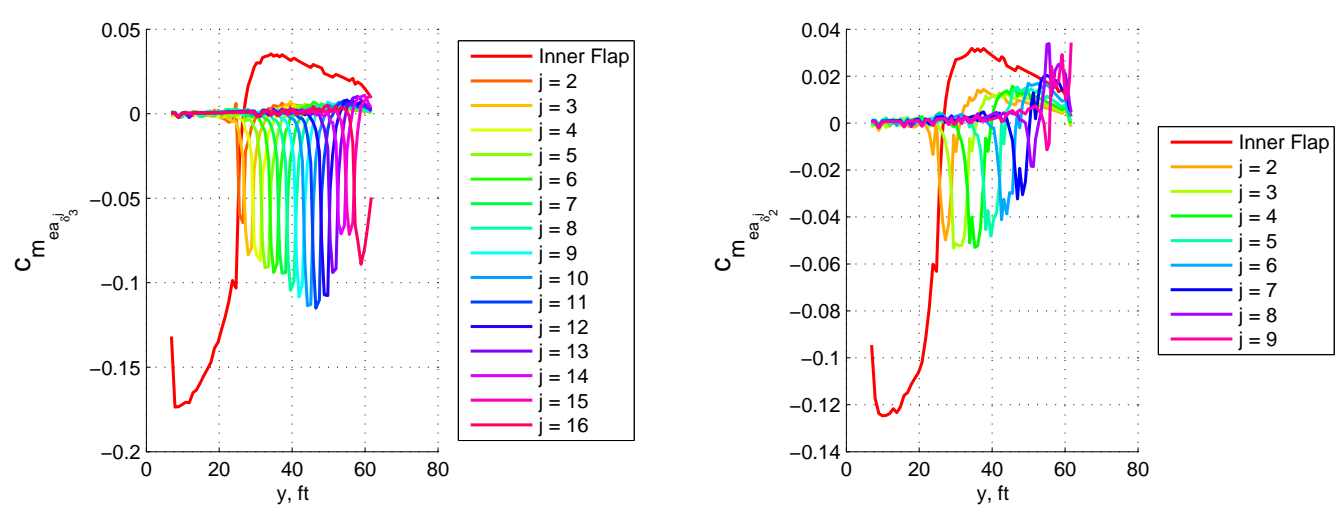

Figure 58. Stiff Wing Low-Speed Take-Off Sectional Pitching Moment (about Elastic Axis) Spanwise Derivatives to VCCTEF, $c_{m_{e a} \delta_{n}}(y)$ (VCCTEF configuration \#1, left, VCCTEF configuration \#2, right)

The total aircraft derivatives with respect to the VCCTEF for the baseline stiff wing GTM are shown in Figs. 59-64. 

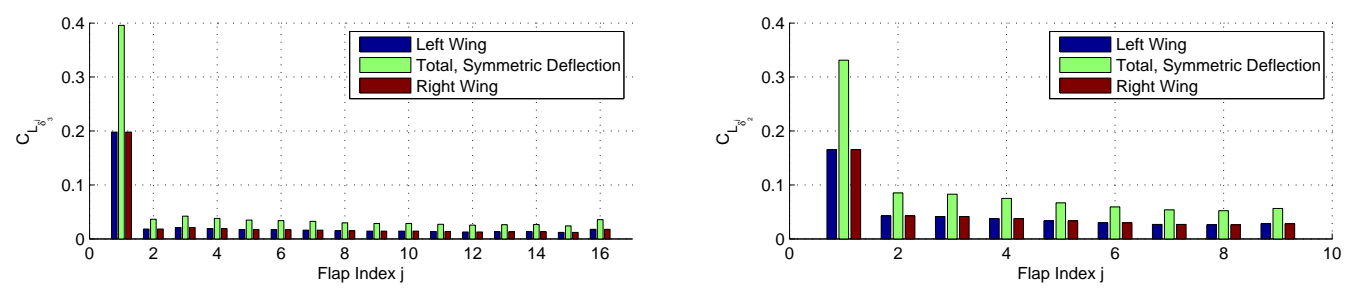

Figure 59. Stiff Wing Low-Speed Take-Off Total Aircraft Lift Derivatives to VCCTEF, $C_{L_{\delta_{n}}}$ (VCCTEF configuration \#1, left, VCCTEF configuration \#2, right)
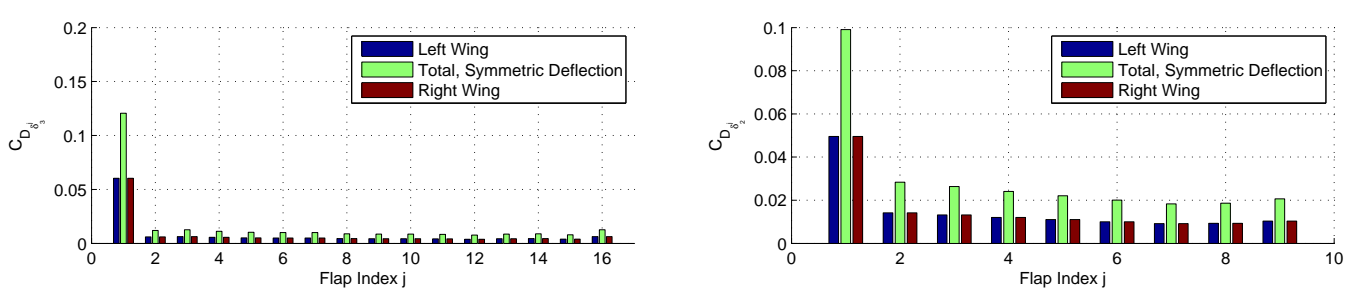

Figure 60. Stiff Wing Low-Speed Take-Off Total Aircraft Drag Derivatives to VCCTEF, $C_{D_{\delta_{n}}}$ (VCCTEF configuration \#1, left, VCCTEF configuration \#2, right)
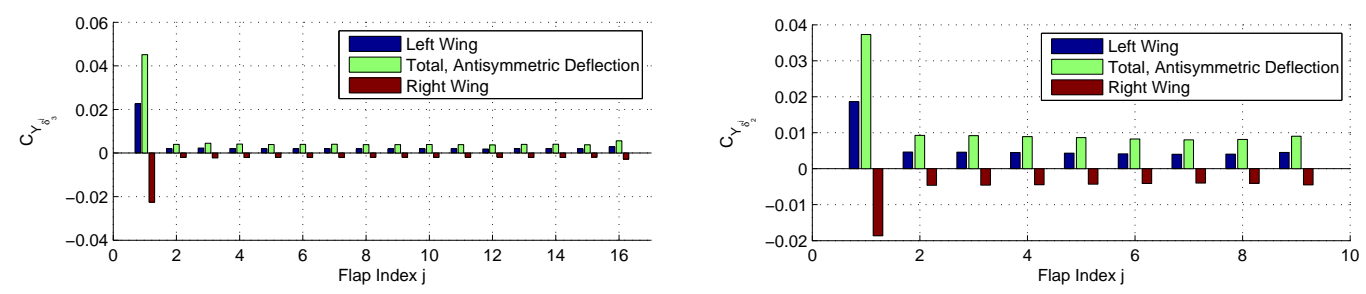

Figure 61. Stiff Wing Low-Speed Take-Off Total Aircraft Side Force Derivatives to VCCTEF, $C_{Y_{\delta_{n}}}$ (VCCTEF configuration \#1, left, VCCTEF configuration \#2, right)
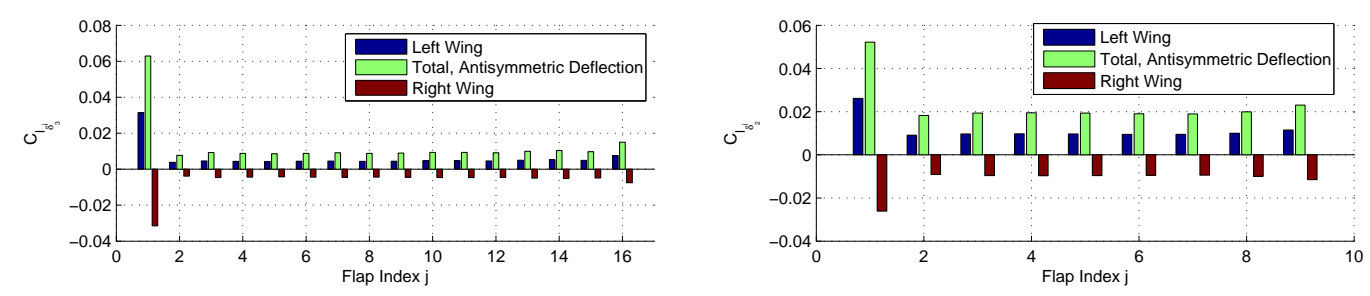

Figure 62. Stiff Wing Low-Speed Take-Off Total Aircraft Rolling Moment Derivatives to VCCTEF, $C_{l_{\delta_{n}}}$ (VCCTEF configuration \#1, left, VCCTEF configuration \#2, right)
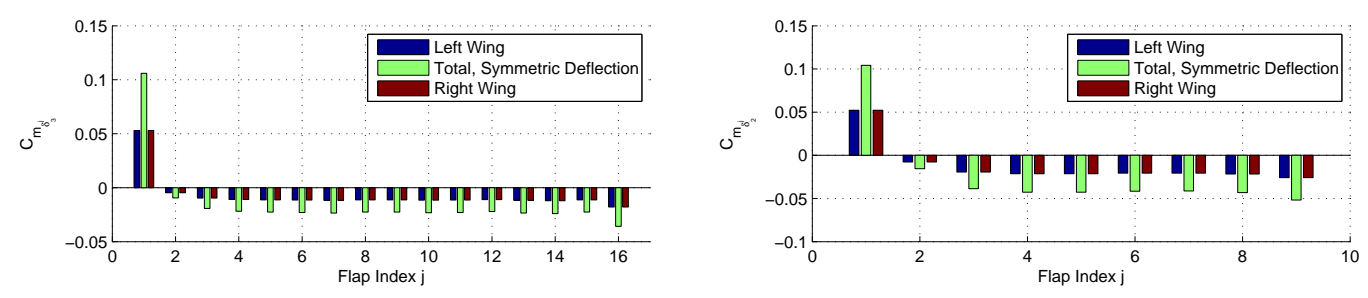

Figure 63. Stiff Wing Low-Speed Take-Off Total Aircraft Pitching Moment Derivatives to VCCTEF, $C_{m_{\delta_{n}}}$ (VCCTEF configuration \#1, left, VCCTEF configuration \#2, right) 

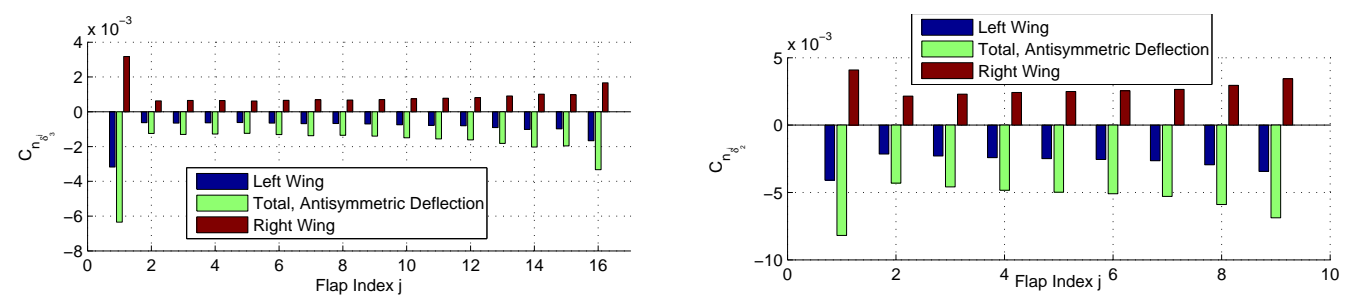

Figure 64. Stiff Wing Low-Speed Take-Off Total Aircraft Yawing Moment Derivatives to VCCTEF, $C_{n_{\delta_{n}}}$ (VCCTEF configuration \#1, left, VCCTEF configuration \#2, right)

FLEXIBLE Wing RESUlTS

Figures 65-68 are the spanwise derivatives with respect to the deflection of the aftmost camber segments of the VCCTEF for the flexible wing model.
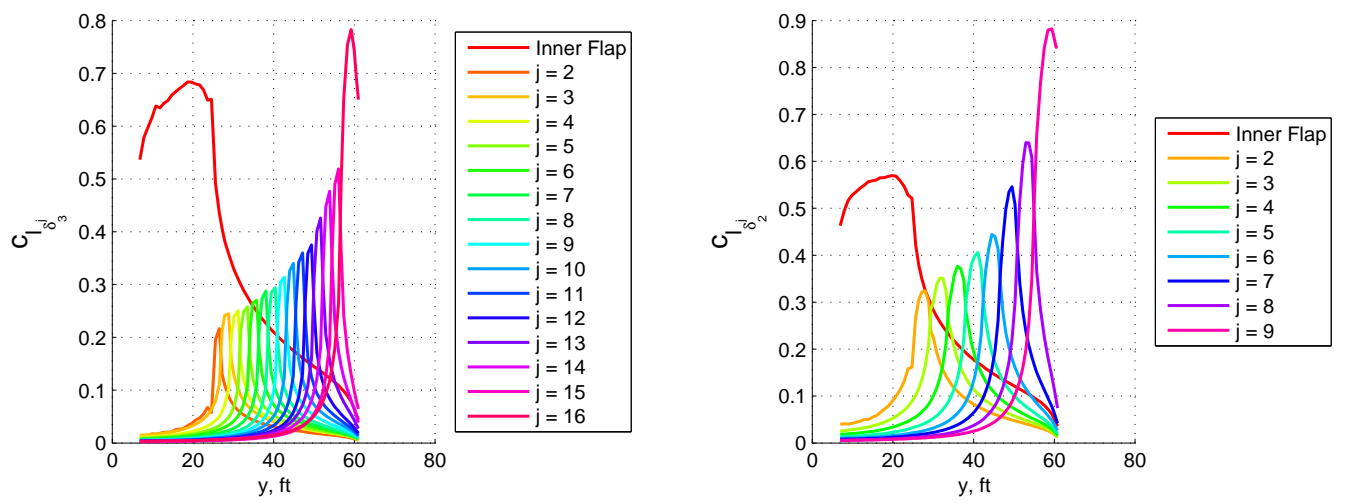

Figure 65. Flexible Wing Low-Speed Take-Off Sectional Lift Spanwise Derivatives to VCCTEF, $c_{l_{\delta_{n}}}(y)$ (VCCTEF configuration \#1, left, VCCTEF configuration \#2, right)
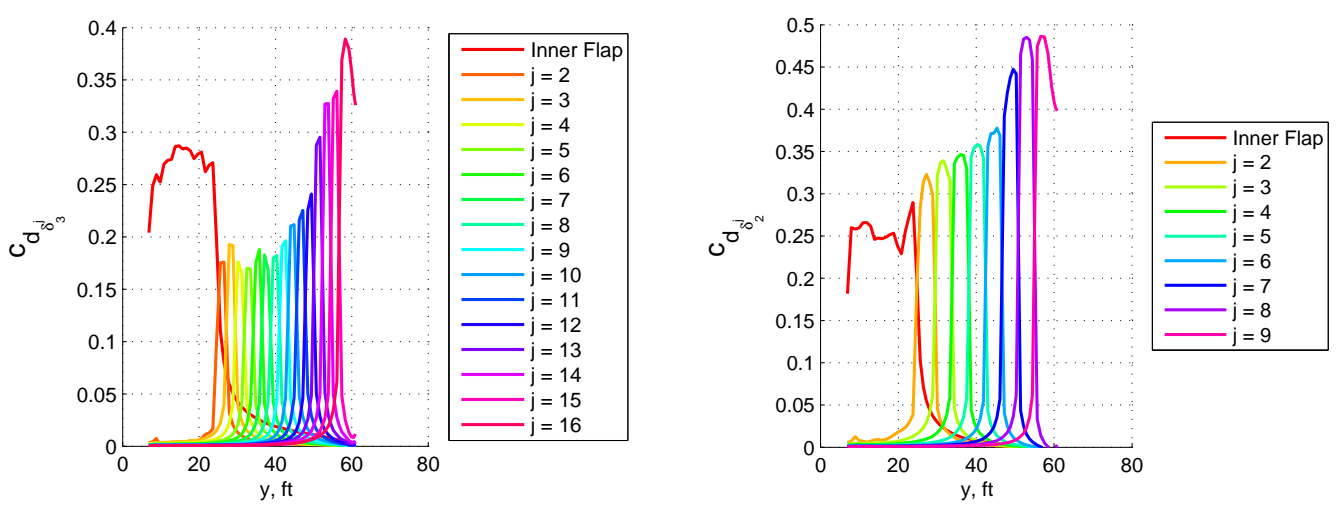

Figure 66. Flexible Wing Low-Speed Take-Off Sectional Drag Spanwise Derivatives to VCCTEF, $c_{d_{\delta_{n}}}(y)$ (VCCTEF configuration \#1, left, VCCTEF configuration \#2, right) 

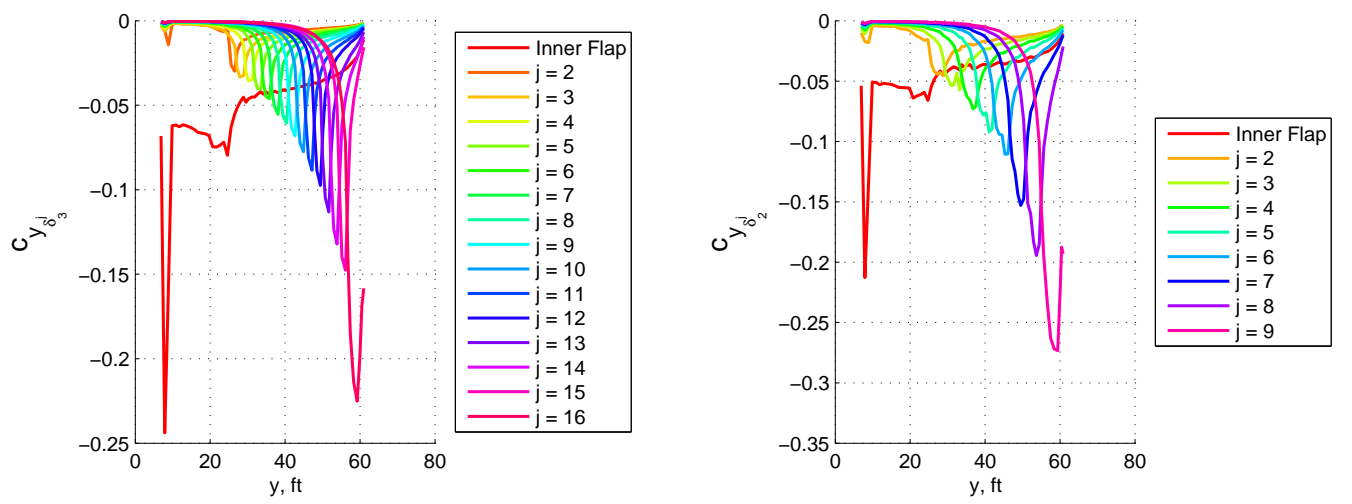

Figure 67. Flexible Wing Low-Speed Take-Off Sectional Side Force Spanwise Derivatives to VCCTEF, $c_{y_{\delta_{n}}}(y)$ (VCCTEF configuration \#1, left, VCCTEF configuration \#2, right)
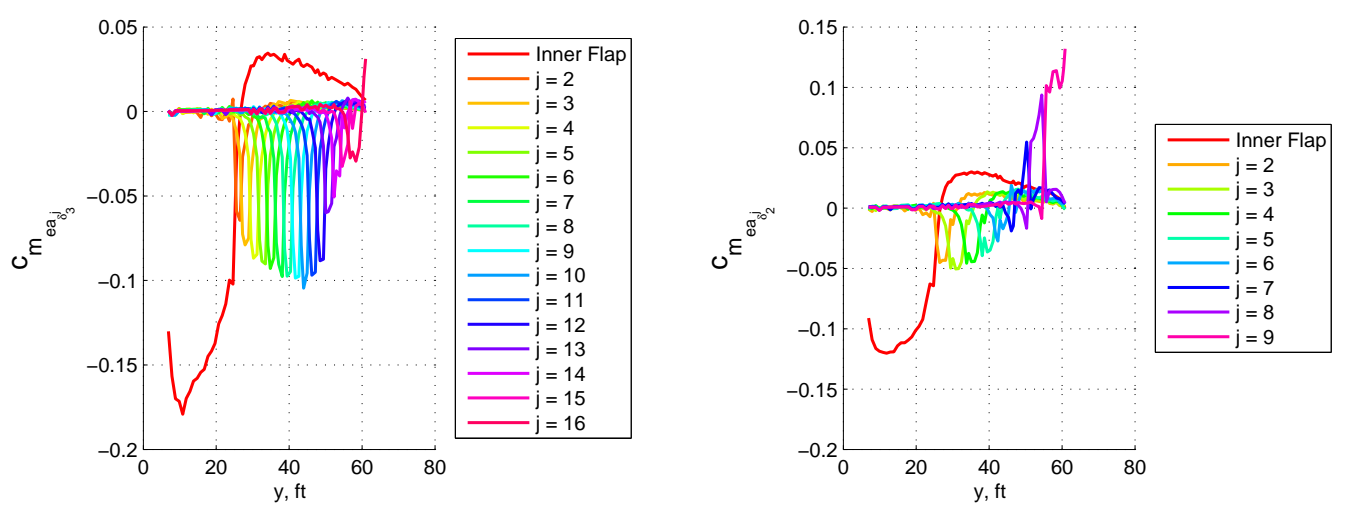

Figure 68. Flexible Wing Low-Speed Take-Off Sectional Pitching Moment (about Elastic Axis) Spanwise Derivatives to VCCTEF, $c_{m_{e} a_{\delta_{n}}}(y)$ (VCCTEF configuration \#1, left, VCCTEF configuration \#2, right)

Figures 69-74 show the total aircraft derivatives with respect to the VCCTEF for the reduced stiffness flexible wing GTM.
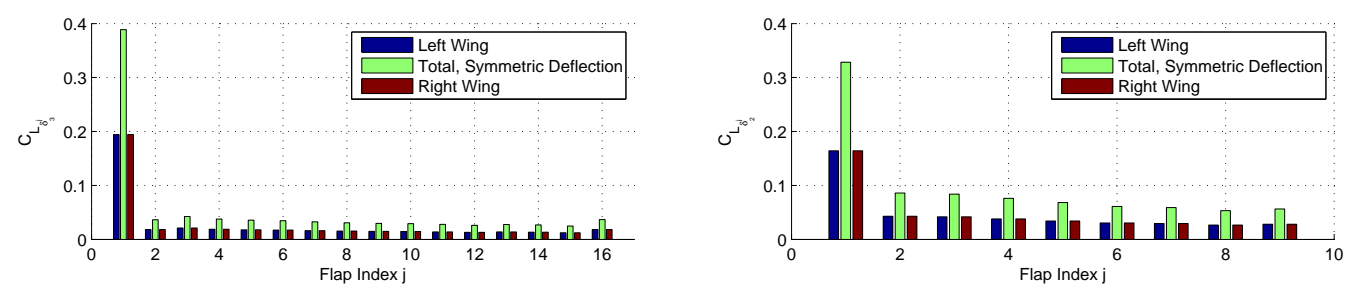

Figure 69. Flexible Wing Low-Speed Take-Off Total Aircraft Lift Derivatives to VCCTEF, $C_{L_{\delta_{n}}}$ (VCCTEF configuration \#1, left, VCCTEF configuration \#2, right) 

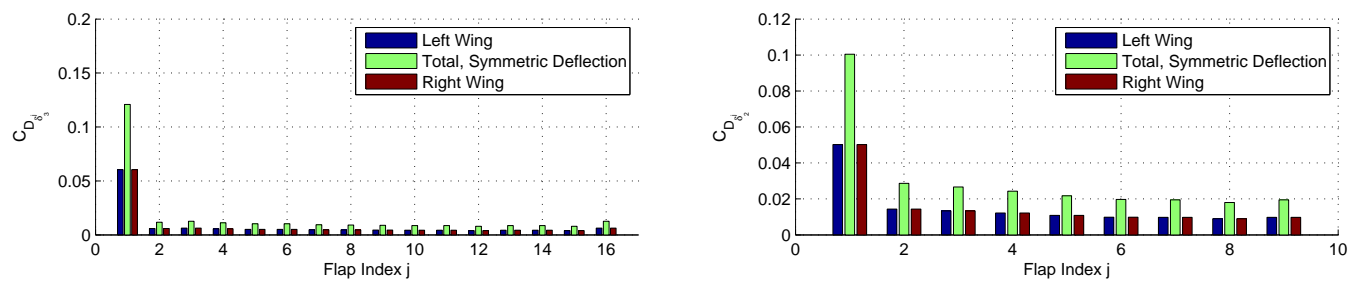

Figure 70. Flexible Wing Low-Speed Take-Off Total Aircraft Drag Derivatives to VCCTEF, $C_{D_{\delta_{n}}}$ (VCCTEF configuration \#1, left, VCCTEF configuration \#2, right)
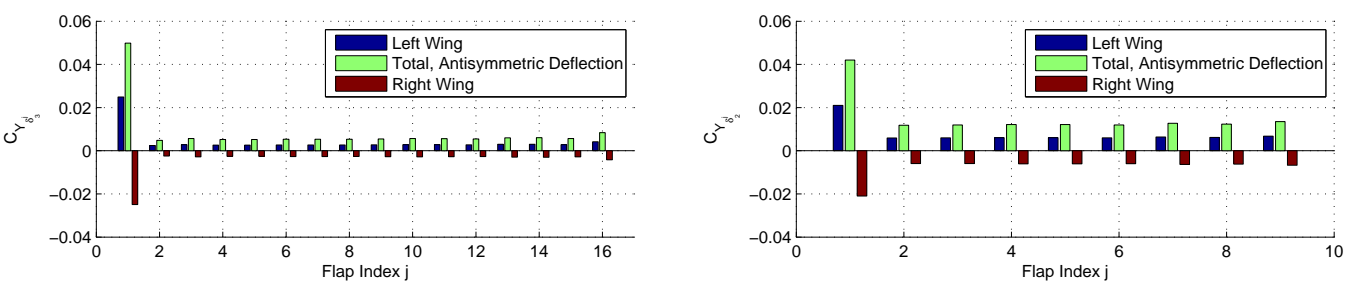

Figure 71. Flexible Wing Low-Speed Take-Off Total Aircraft Side Force Derivatives to VCCTEF, $C_{Y_{\delta_{n}}}$ (VCCTEF configuration \#1, left, VCCTEF configuration \#2, right)
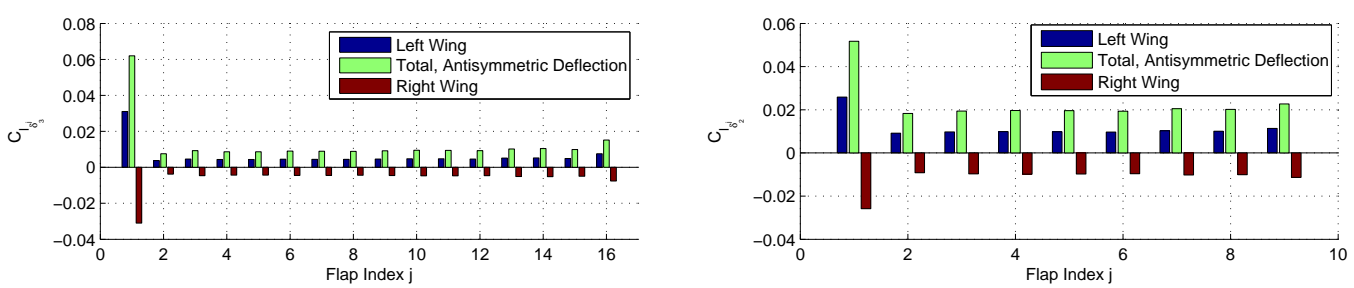

Figure 72. Flexible Wing Low-Speed Take-Off Total Aircraft Rolling Moment Derivatives to VCCTEF, $C_{l_{\delta_{n}}}$ (VCCTEF configuration \#1, left, VCCTEF configuration \#2, right)
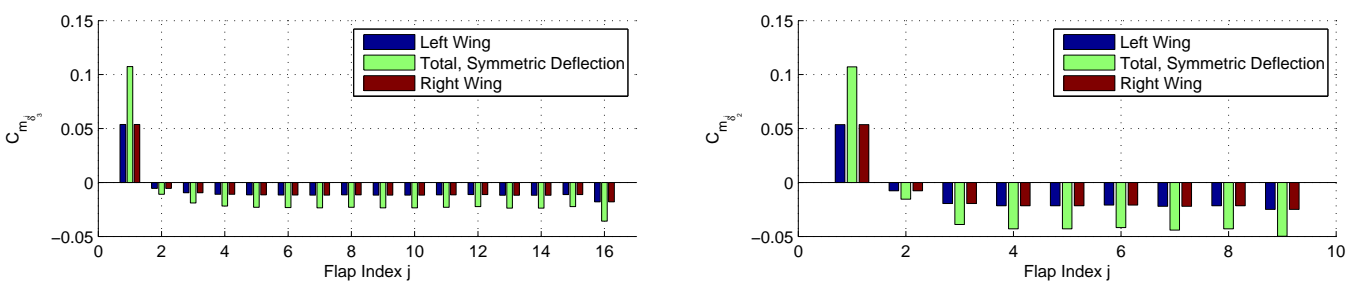

Figure 73. Flexible Wing Low-Speed Take-Off Total Aircraft Pitching Moment Derivatives to VCCTEF, $C_{m_{\delta_{n}}}$ (VCCTEF configuration \#1, left, VCCTEF configuration \#2, right)
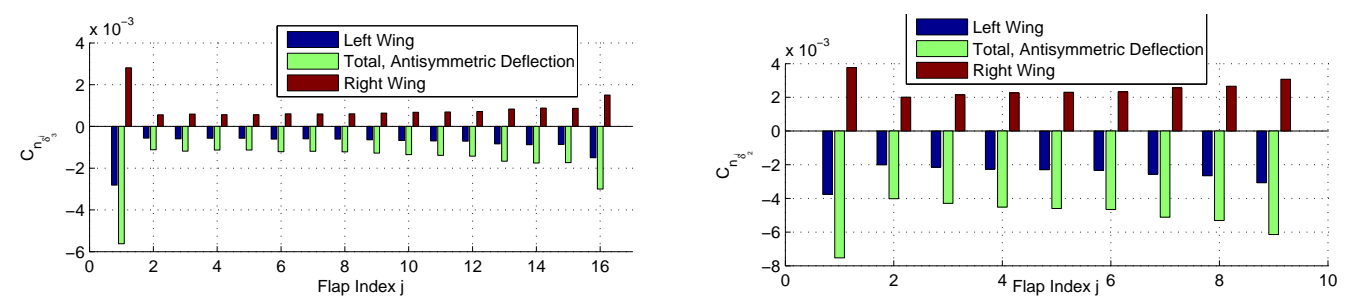

Figure 74. Flexible Wing Low-Speed Take-Off Total Aircraft Yawing Moment Derivatives to VCCTEF, $C_{n_{\delta_{n}}}$ (VCCTEF configuration \#1, left, VCCTEF configuration \#2, right) 


\section{LOW-SpeEd TAKe-OFF CONTROL Derivative Summary}

The total aircraft derivatives for the fast actuated portion of the VCCTEF used for maneuvering at the low-speed take-off flight condition are summarized in Table 4.

\begin{tabular}{|c|c|c|c|c|c|c|c|}
\hline Aircraft Configuration & $C_{L_{\delta_{f, s}}}$ & $C_{D_{\delta_{f, s}}}$ & $C_{D_{\left(\delta_{f, s}\right)^{2}}}$ & $C_{Y_{\delta_{f, a}}}\left(C_{\delta_{\delta a}}\right)$ & $C_{l_{\delta_{f, a}}}\left(C_{l_{\delta a}}\right)$ & $C_{m_{\delta_{f, s}}}$ & $C_{n_{\delta_{f, a}}}\left(C_{n_{\delta a}}\right)$ \\
\hline $\begin{array}{c}\text { Rigid Wing } \\
\text { VCCTEF Configuration \#1 }\end{array}$ & 0.4526 & 0.1443 & -0.4268 & 0.0368 & 0.1378 & -0.3340 & -0.0264 \\
\hline $\begin{array}{c}\text { Rigid Wing } \\
\text { VCCTEF Configuration \#2 }\end{array}$ & 0.5134 & 0.1783 & -0.1807 & 0.0406 & 0.1514 & -0.3105 & -0.0444 \\
\hline $\begin{array}{c}\text { Stiff Wing } \\
\text { VCCTEF Configuration \#1 }\end{array}$ & 0.4704 & 0.1463 & -0.3776 & 0.0611 & 0.1429 & -0.3389 & -0.0243 \\
\hline $\begin{array}{c}\text { Stiff Wing } \\
\text { VCCTEF Configuration \#2 }\end{array}$ & 0.5314 & 0.1783 & -0.2791 & 0.0694 & 0.1571 & -0.3174 & -0.0419 \\
\hline $\begin{array}{c}\text { Flexible Wing } \\
\text { VCCTEF Configuration \#1 }\end{array}$ & 0.4787 & 0.1466 & -0.0657 & 0.0850 & 0.1442 & -0.3421 & -0.0213 \\
\hline $\begin{array}{c}\text { Flexible Wing } \\
\text { VCCTEF Configuration \#2 }\end{array}$ & 0.5440 & 0.1781 & -0.4597 & 0.0985 & 0.1597 & -0.3191 & -0.0386 \\
\hline
\end{tabular}

Table 4. Control Derivative Values for VCCTEF Maneuvering Sections at Low-Speed Take-Off

Similar trends are observed in Table 4 and the figures from the control derivatives analyses show similar trends as were observed from the cruise condition results. The lift coefficient and roll control coefficient derivatives $C_{L_{\delta}}$ and $C_{l_{\delta}}$ both increase as wing flexibility increases. However, the first order drag control coefficient $C_{D_{\delta}}$ does not observe the same trend as that of the cruise condition results in Table 3, which showed a decreasing trend of $C_{D_{\delta}}$ as wing flexibility increases.

\section{Hinge Moment Analysis}

A hinge moment analysis is conducted using vortex-lattice data to approximate the loads on the VCCTEF at given flight conditions.

\section{A. Methodology}

Hinge moment data is obtained by using $\Delta c_{p}$ pressure data that is obtained from running vortex-lattice. Given that vortex-lattice returns $\Delta c_{p}(x, y)$ from Eq. 11, determining the normal load experienced at each hinge requires integrating the pressure difference aft of the hinge line location. Letting the global spanwise direction be the independent variable, the hinge line locations are given by the axis $\left(x_{h_{k}}, y_{h_{k}}, z_{h_{k}}\right)$ or $\left(x_{h_{k}}(y), z_{h_{k}}(y)\right)$. Thus, the normal load coefficient can be determined through integration as follows

$$
c_{n, h_{k}}(y)=\frac{1}{c(y)} \int_{x_{h_{k}}(y)}^{x_{t e}(y)} \Delta c_{p}(x, y) d x
$$

where $x_{t e}(y)$ is the location of the trailing edge. The moment coefficient at hinge location about the panel $v$-axis is

$$
c_{m, h_{k}}(y)=\frac{1}{c(y)^{2}} \int_{x_{h_{k}}(y)}^{x_{t e}(y)}-\Delta c_{p}(x, y)\left(x-x_{h_{k}}(y)\right) d x
$$

for the $k$-th camber segment. Note the coefficients reference the wing sectional chord $c(y)$. The hinge normal load and moment can be transformed to the global directions using Eq. 6: 


$$
\begin{gathered}
{\left[\begin{array}{l}
c_{x, h} \\
c_{y, h} \\
c_{z, h}
\end{array}\right]=\left[\begin{array}{ccc}
\cos \gamma & 0 & -\sin \gamma \\
-\sin \gamma \sin \Gamma & \cos \Gamma & -\cos \gamma \sin \Gamma \\
\sin \gamma \cos \Gamma & \sin \Gamma & \cos \gamma \cos \Gamma
\end{array}\right]\left[\begin{array}{c}
0 \\
0 \\
c_{n, h}
\end{array}\right]=\left[\begin{array}{c}
-c_{n, h} \sin \gamma \\
-c_{n, h} \cos \gamma \sin \Gamma \\
c_{n, h} \cos \gamma \cos \Gamma
\end{array}\right]} \\
{\left[\begin{array}{c}
c_{m_{x}, h} \\
c_{m_{y}, h} \\
c_{m_{z}, h}
\end{array}\right]=\left[\begin{array}{ccc}
\cos \gamma & 0 & -\sin \gamma \\
-\sin \gamma \sin \Gamma & \cos \Gamma & -\cos \gamma \sin \Gamma \\
\sin \gamma \cos \Gamma & \sin \Gamma & \cos \gamma \cos \Gamma
\end{array}\right]\left[\begin{array}{c}
0 \\
c_{m, h} \\
0
\end{array}\right]=\left[\begin{array}{c}
0 \\
c_{m, h} \cos \Gamma \\
c_{m, h} \sin \Gamma
\end{array}\right]}
\end{gathered}
$$

The hinge line axis is not a straight line, especially when aeroelastic deformation of the wing is included. Thus, to determine the hinge moment on a VCCTEF hinge section/segment, it is necessary to take the projection of moment coefficient along the hinge line directions. Let the direction of the hinge line be represented by

$$
\mathbf{s}_{h, k}=\frac{1}{\left\|\sqrt{1+\left(\frac{d x_{h, k}}{d y}\right)^{2}+\left(\frac{d z_{h, k}}{d y}\right)^{2}}\right\|}\left\langle\frac{d x_{h, k}}{d y}, 1, \frac{d z_{h, k}}{d y}\right\rangle
$$

The distributed hinge moment load can then be determined by calculating the dot product between the hinge moment coefficients in the global directions with the hinge line directions

$$
\begin{aligned}
m_{h}(y) & =q_{\infty} c(y)^{2}\left\langle 0, c_{m, h}(y) \cos \Gamma(y), c_{m, h}(u) \sin \Gamma(y)\right\rangle \cdot \mathbf{s}_{h}(y) \\
& =\frac{q_{\infty} c(y)^{2}}{\left\|\sqrt{1+\left(\frac{d x_{h}(y)}{d y}\right)^{2}+\left(\frac{d z_{h}(y)}{d y}\right)^{2}}\right\|}\left(c_{m, h}(y) \cos \Gamma(y)+c_{m, h}(y) \sin \Gamma(y) \frac{d z_{h}(y)}{d y}\right)
\end{aligned}
$$

The hinge moment load over the $j$-th flap section for the $k$-th camber section is the result of integrating the distributed hinge moment load over the independent global spanwise direction

$$
M_{h_{k}^{j}}=c_{m, h_{k}^{j}} q_{\infty} S \bar{c}=\int_{y_{1}^{j}}^{y_{2}^{j}} m_{h_{k}}(y) d y
$$

where $y_{1}^{j}$ and $y_{2}^{j}$ are the spanwise coordinates for the edge of the VCCTEF flap sections where $y_{2}^{j}>y_{1}^{j}$.

\section{B. Coordinated Turn Maneuver}

In order to examine flight conditions where the VCCTEF is deflected for maneuvers, steady state coordinated turn maneuvers are simulated in order to approximate a loaded flight condition. During the steady state coordinated turn, the aircraft airspeed and altitude are not changing while the aircraft maintains a constant yaw rate. The aircraft tracks both a yaw rate and bank angle command during the coordinated turn. The linearized heading equation for the aircraft is given by

$$
\dot{\psi}=r \sec \bar{\theta}
$$

where for small angles $\dot{\psi}=r$. The commands for a steady state coordinated turn are

$$
\begin{gathered}
r_{c}=\frac{g \sqrt{n^{2}-1}}{\bar{V}} \\
\phi_{c}=\cos ^{-1} \frac{1}{n}
\end{gathered}
$$

where $n$ is the aircraft load factor. The necessary VCCTEF deflections needed to conduct the coordinated turn can be solved for by utilizing the linearized lateral-direction dynamics for the aircraft. The complete formulation can be found in reference. ${ }^{4}$ The steady roll and yaw equations are given by

$$
-\frac{L_{\beta} g}{Y_{\beta}} \phi_{c}+\left(L_{r}-\frac{L_{\beta}\left(Y_{r}-\bar{u}\right)}{Y_{\beta}}\right) r_{c}+\left(L_{\delta_{f}}-\frac{L_{\beta} Y_{\delta_{f}}}{Y_{\beta}}\right) \delta_{f}+\left(L_{\delta r}-\frac{L_{\beta} Y_{\delta r}}{Y_{\beta}}\right) \delta r=0
$$




$$
-\frac{N_{\beta} g}{Y_{\beta}} \phi_{c}+\left(N_{r}-\frac{N_{\beta}\left(Y_{r}-\bar{u}\right)}{Y_{\beta}}\right) r_{c}+\left(N_{\delta_{f}}-\frac{N_{\beta} Y_{\delta_{f}}}{Y_{\beta}}\right) \delta_{f}+\left(N_{\delta r}-\frac{N_{\beta} Y_{\delta r}}{Y_{\beta}}\right) \delta r=0
$$

where $\delta_{f}$ and $\delta r$ are solved for given $r_{c}$ and $\phi_{c}$ as a function of the load factor $n$.

It is assumed that the terms $\frac{L_{\beta} Y_{\delta_{f}}}{Y_{\beta}}, N_{\delta_{f}}$, and $\frac{N_{\beta} Y_{\delta_{f}}}{Y_{\beta}}$ are small, thus reducing Eqs. 52 and 53 to

$$
\begin{gathered}
-L_{\beta} g \phi_{c}+\left(L_{r} Y_{\beta}-L_{\beta}\left(Y_{r}-\bar{u}\right)\right) r_{c}+L_{\delta_{f}} Y_{\beta} \delta_{f}+\left(L_{\delta r} Y_{\beta}-L_{\beta} Y_{\delta r}\right) \delta r=0 \\
-N_{\beta} g \phi_{c}+\left(N_{r} Y_{\beta}-N_{\beta}\left(Y_{r}-\bar{u}\right)\right) r_{c}+\left(N_{\delta r} Y_{\beta}-N_{\beta} Y_{\delta r}\right) \delta r=0
\end{gathered}
$$

where $L_{\beta}=\frac{C_{l_{\beta}} q_{\infty} S b}{I_{x x}}, L_{r}=\frac{C_{l_{r}} q_{\infty} S b^{2}}{2 I_{x x} \bar{u}}, L_{\delta_{f}}=\frac{C_{l_{\delta_{f}}} q_{\infty} S b}{I_{x x}}, L_{\delta r}=\frac{C_{l_{\delta_{r}}} q_{\infty} S b}{I_{x x}}, Y_{\beta}=\frac{C_{Y_{\beta}} q_{\infty} S}{m}, Y_{r}=\frac{C_{Y_{r}} q_{\infty} S b}{2 m \bar{u}}, Y_{\delta_{f}}=\frac{C_{Y_{\delta_{f}}} q_{\infty} S}{m}, Y_{\delta r}=$ $\frac{C_{Y_{\delta}} q_{\infty} S}{m}, N_{\beta}=\frac{C_{n_{\beta}} q_{\infty} S b}{I_{z z}}, N_{r}=\frac{C_{n_{r}} q_{c_{\infty} S b^{2}}}{2 I_{z z} \bar{u}}, N_{\delta_{f}}=\frac{C_{n_{\delta_{f}}} q_{\infty} S b}{I_{z z}}, N_{\delta r}=\frac{C_{n_{\delta r}} q_{\infty} S}{I_{z z}}, m=210,000 / 32.174$ slug for cruise, $m=$ $235,000 / 32.174$ slug for low-speed take-off, $I_{x x}=1,770,000$ slug $-\mathrm{ft}^{2}$, and $I_{z z}=7,270,000 \mathrm{slug}-\mathrm{ft}^{2}{ }^{11}$ Thus Eqs. 54 and 55 are used to solve for $\delta_{f}$ and $\delta r$ for the coordinated turn maneuvers.

\section{Results}

To estimate the worst case hinge moment loading that the VCCTEF will encounter, the hinge moment coefficients for the VCCTEF flap sections are determined by considering the cruise and the low-speed take-off flight conditions. Three subcases are run for the cruise flight condition: cruise steady state, cruise at the maximum maneuver load, and a coordinated turn maneuver conducted with control deflection limits. For the low-speed take-off flight condition, the same three subcases are considered.

For cruise, the maximum angle of attack for the aircraft is considered $\alpha_{\text {stall }}=12^{\circ}$. For low-speed take-off, the maximum angle of attack for the aircraft is considered to be slightly lower at $\alpha_{\text {stall }}=10^{\circ}$ due to the high-lift flap deflections. The maximum rudder deflection is set at $\delta r=10^{\circ}$ and the maximum limit on the VCCTEF maneuver sections is considered to be $\delta_{f}=30^{\circ}$.

\section{Cruise}

The results for the three subcases at the cruise condition of $M=0.797, h=36,000 \mathrm{ft}$ are presented.

\section{CRuise Steady STATE}

The cruise steady state condition is considered for standard flight at $C_{L}=0.51$ with $\bar{\delta}=0^{\circ}$. The flight conditions for the rigid, stiff, and flexible wing aircraft at the cruise steady state were presented earlier in Table 1. For those flight conditions, the distributed hinge moment loads $m_{h}(y)$ are shown in Fig. 75 and the integrated hinge moment loads $M_{h}$ are shown in Fig. 76.
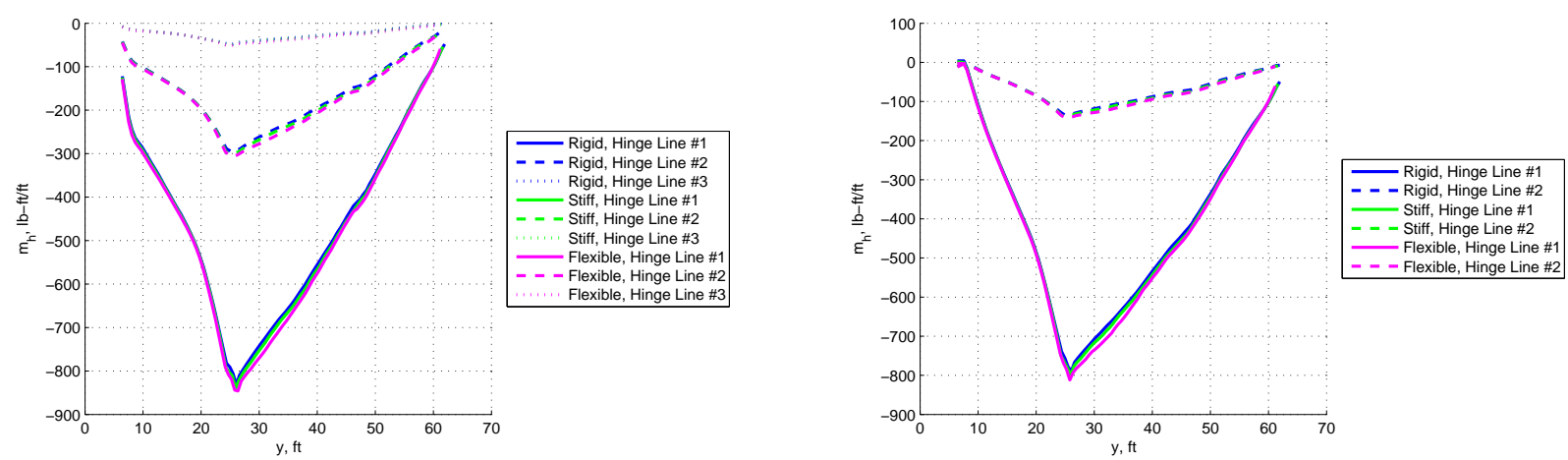

Figure 75. Distributed VCCTEF Hinge Moment Loads $m_{h}(y)$ at Cruise Steady State (VCCTEF configuration \#1, left, VCCTEF configuration \#2, right) 

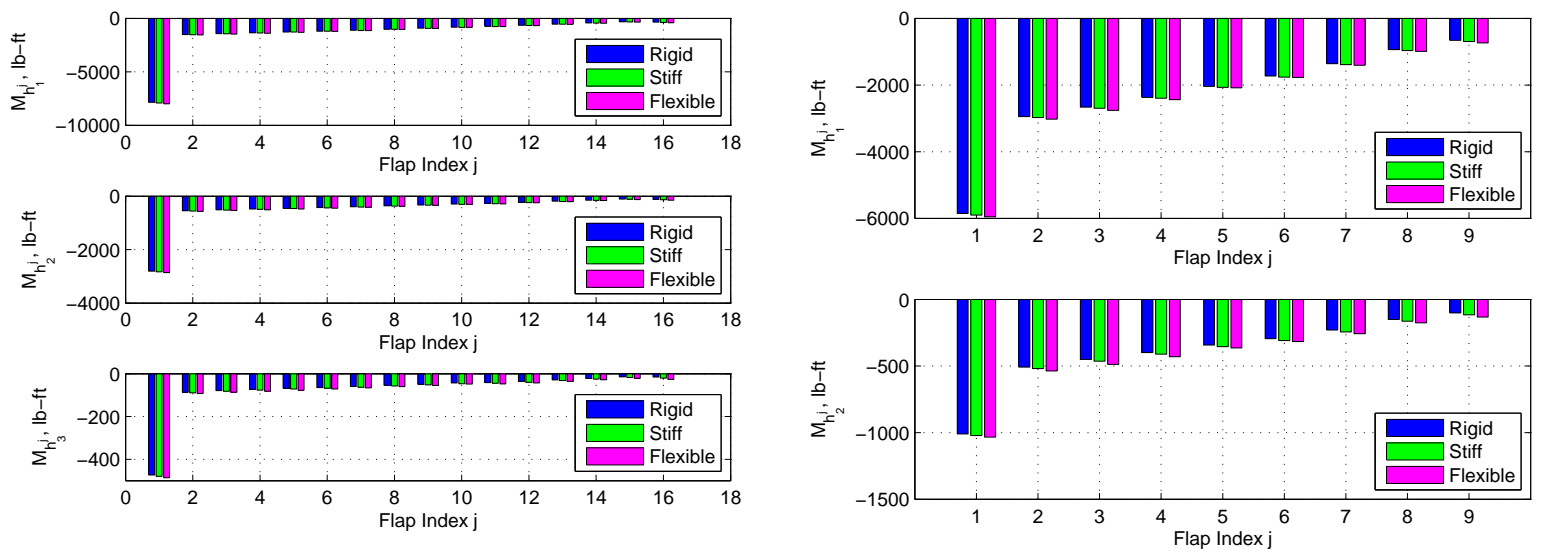

Figure 76. Integrated VCCTEF Hinge Moments $M_{h}$ at Cruise Steady State (VCCTEF configuration \#1, left, VCCTEF configuration \#2, right)

\section{CRUise AT MAXIMUM MANEUVER LOAD}

The cruise maximum maneuver load hinge moment analysis determines the VCCTEF hinge moment coefficients at the cruise speed and altitude where the load factor has been increased to the positive maximum $n=2.5$, or the angle of attack is increased to $\alpha=12^{\circ}$. The static aeroelastic model solutions for the loaded flight condition are summarized in Table 5. The flexible wing configuration is angle of attack limited.

\begin{tabular}{|c|c|c|c|c|c|c|}
\hline \multicolumn{7}{|c|}{ VCCTEF Configuration \#1 } \\
\hline & $\alpha(\mathrm{deg})$ & $n$ & $C_{L}$ & $W_{\text {tip }}(\mathrm{ft})$ & $\Theta_{\text {tip }}(\mathrm{deg})$ & $\Delta \phi_{y, t i p}(\mathrm{deg})$ \\
\hline \hline Rigid Wing & 9.945 & 2.50 & 1.28 & - & - & - \\
\hline Stiff Wing & 11.133 & 2.50 & 1.28 & 5.998 & -2.586 & -1.817 \\
\hline Flexible Wing & 12.000 & 2.41 & 1.23 & 10.818 & -4.407 & -3.418 \\
\hline
\end{tabular}

\begin{tabular}{|c|c|c|c|c|c|c|}
\hline \multicolumn{7}{|c|}{ VCCTEF Configuration \#2 } \\
\hline & $\alpha(\mathrm{deg})$ & $n$ & $C_{L}$ & $W_{\text {tip }}(\mathrm{ft})$ & $\Theta_{\text {tip }}(\mathrm{deg})$ & $\Delta \phi_{\text {y,tip }}(\mathrm{deg})$ \\
\hline \hline Rigid Wing & 9.914 & 2.50 & 1.28 & - & - & - \\
\hline Stiff Wing & 11.157 & 2.50 & 1.28 & 6.095 & -2.569 & -1.938 \\
\hline Flexible Wing & 12.000 & 2.40 & 1.23 & 10.934 & -4.341 & -3.618 \\
\hline
\end{tabular}

Table 5. Static Aeroelastic Solutions for Loaded Steady State Cruise, $M=0.797, h=36000$ ft, $C_{L}=0.51$

Figures 77 and 78 show the the distributed hinge moment loads $m_{h}(y)$ and the integrated hinge moment loads for the loaded flight condition. 

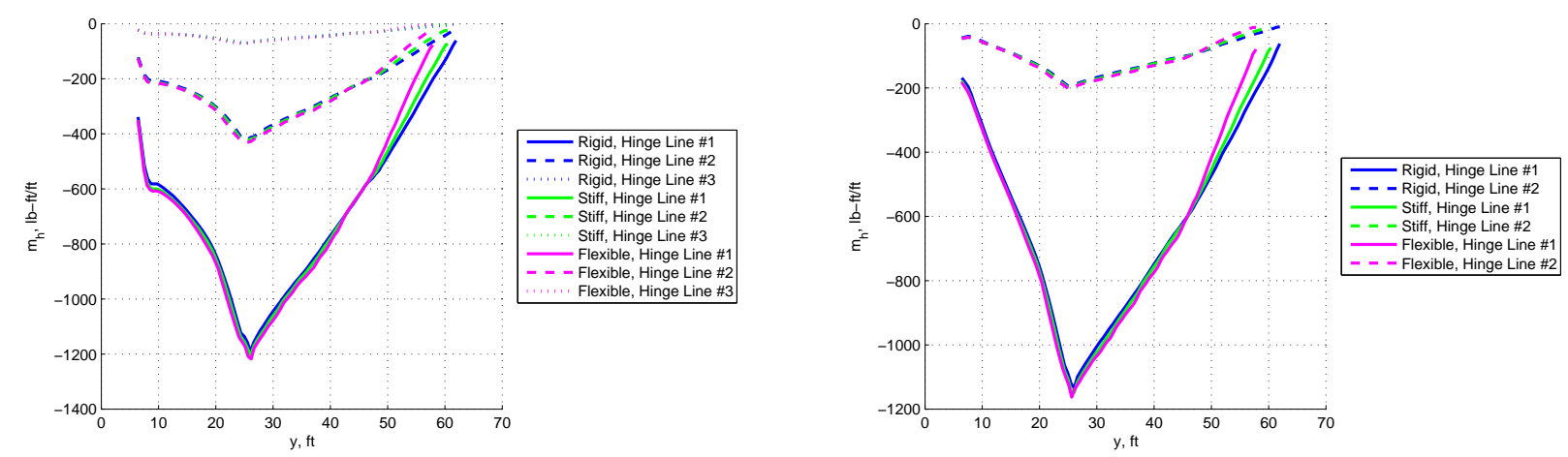

Figure 77. Distributed VCCTEF Hinge Moment Loads $m_{h}(y)$ at Loaded Cruise (VCCTEF configuration \#1, left, VCCTEF configuration \#2, right)
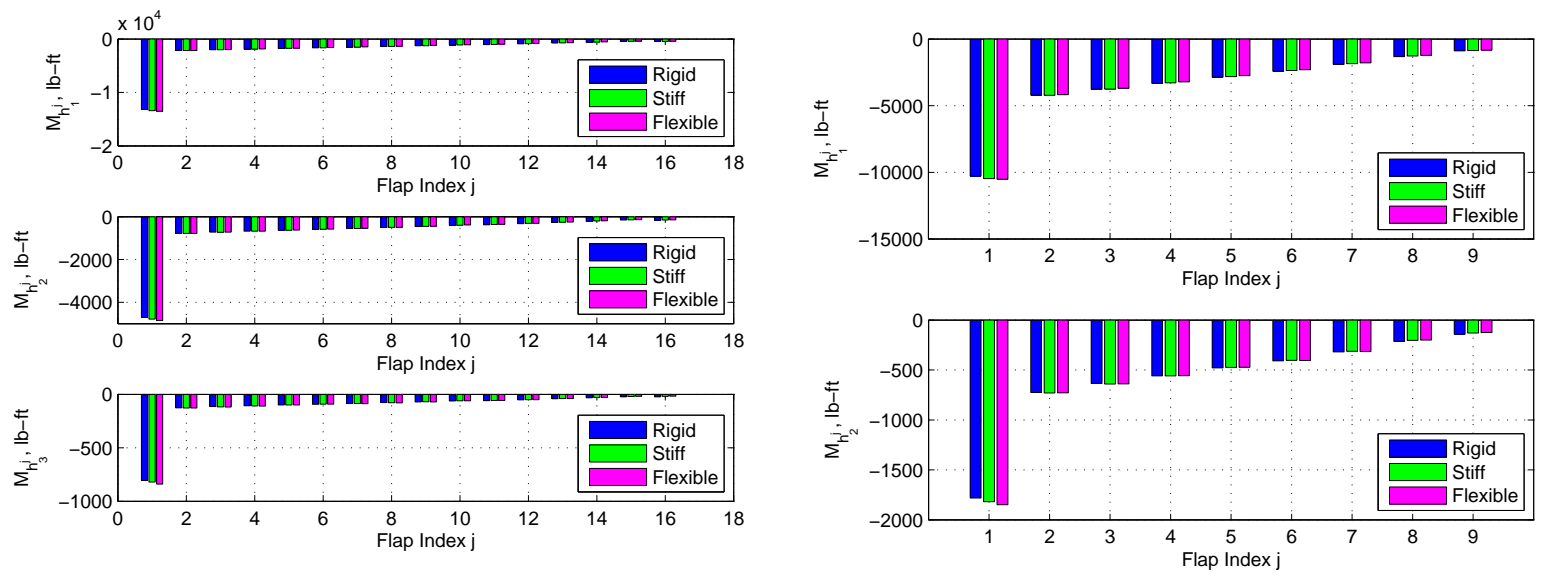

Figure 78. Integrated VCCTEF Hinge Moments $M_{h}$ at Loaded Cruise (VCCTEF configuration \#1, left, VCCTEF configuration \#2, right)

\section{CRUise With Limited COORDinATED TURN MANEUVER}

The hinge moments at cruise when the aircraft is conducting a coordinated turn take into account the control deflections necessary to execute the maneuver. Antisymmetric deflection of the VCCTEF is used to roll the aircraft. Deflection of the maneuver segments of the VCCTEF is assumed to have no impact on the aeroelastic shape of the wing, and the net effect of the antisymmetric deployment of the VCCTEF on the aircraft $C_{L}$ is neglected. The static aeroelastic model is run for the loaded flight condition, and the VCCTEF flap deflections are calculated using the VCCTEF roll derivatives at the flexible wing shape. The aircraft configuration for the coordinated turn maneuver is summarized in Table 6. Maneuvering VCCTEF deflection limits and rudder deflection limits are imposed. 


\begin{tabular}{|c|c|c|c|c|c|c|c|c|c|}
\hline \multicolumn{10}{|c|}{ VCCTEF Configuration \#1 } \\
\hline & $\alpha(\mathrm{deg})$ & $n$ & $C_{L}$ & $W_{\text {tip }}(\mathrm{ft})$ & $\Theta_{\text {tip }}(\mathrm{deg})$ & $\Delta \phi_{y, t i p}(\mathrm{deg})$ & $C_{l_{\delta_{f}}}$ & $\delta_{f}$ & $\delta r$ \\
\hline \hline Rigid Wing & 4.693 & 1.41 & 0.72 & - & - & - & 0.3018 & 5.687 & 10.001 \\
\hline Stiff Wing & 5.297 & 1.41 & 0.72 & 3.123 & -0.934 & -1.364 & 0.2954 & 6.037 & 10.000 \\
\hline Flexible Wing & 5.900 & 1.41 & 0.72 & 5.892 & -1.504 & -2.767 & 0.2849 & 6.492 & 10.000 \\
\hline
\end{tabular}

\begin{tabular}{|c|c|c|c|c|c|c|c|c|c|}
\hline \multicolumn{10}{|c|}{ VCCTEF Configuration \#2 } \\
\hline & $\alpha(\mathrm{deg})$ & $n$ & $C_{L}$ & $W_{\text {tip }}(\mathrm{ft})$ & $\Theta_{\text {tip }}(\mathrm{deg})$ & $\Delta \phi_{y, t i p}(\mathrm{deg})$ & $C_{l_{\delta_{f}}}$ & $\delta_{f}$ & $\delta r$ \\
\hline \hline Rigid Wing & 4.687 & 1.41 & 0.72 & - & - & - & 0.3330 & 5.151 & 10.000 \\
\hline Stiff Wing & 5.309 & 1.41 & 0.72 & 3.189 & -0.922 & -1.437 & 0.3258 & 5.479 & 9.999 \\
\hline Flexible Wing & 5.931 & 1.41 & 0.72 & 5.988 & -1.462 & -2.912 & 0.3139 & 5.902 & 9.999 \\
\hline
\end{tabular}

Table 6. Static Aeroelastic Solutions for Loaded Turn Maneuver at Cruise, $M=0.797, h=36000$ ft, $C_{L}=0.51$

Table 6 shows that all three of the rigid, stiff, and flexible configurations are rudder deflection limited. For the loaded turn maneuver case, hinge moment data is plotted for the left wing with positive VCCTEF maneuver flap deflection, which is higher than that of the hinge moment data for the right with negative VCCTEF maneuver section deflection. The values for the distributed hinge moments loads and the integrated values for each flap section are shown in Figures 79 and 80.
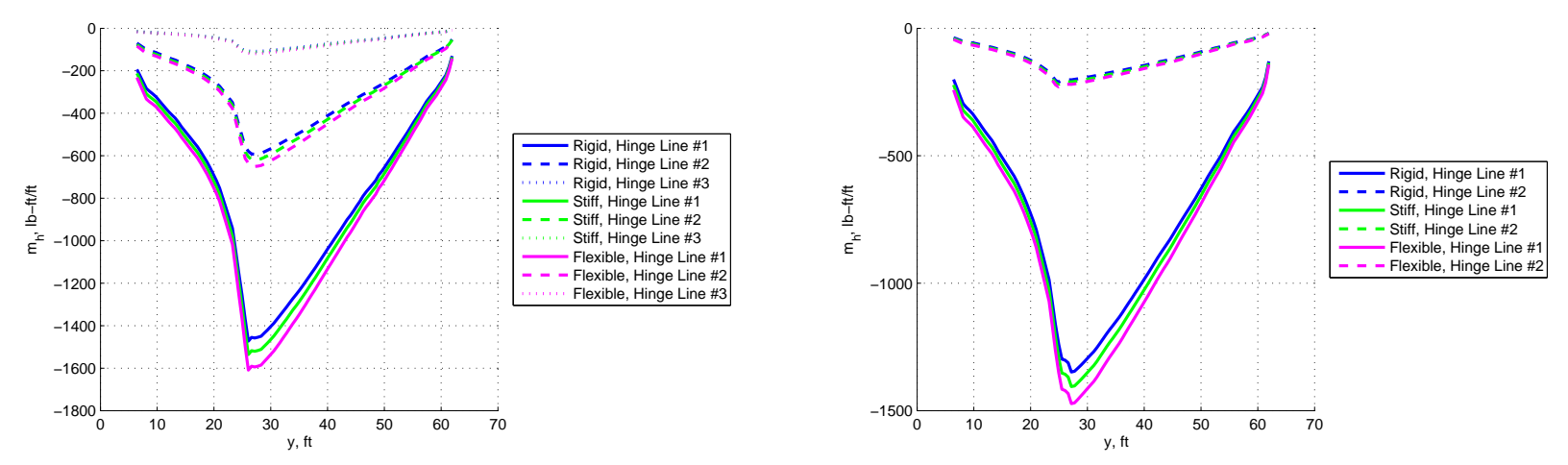

Figure 79. Distributed VCCTEF Hinge Moment Loads $m_{h}(y)$ for Loaded Turn Maneuver at Cruise (VCCTEF configuration \#1, left, VCCTEF configuration \#2, right) 

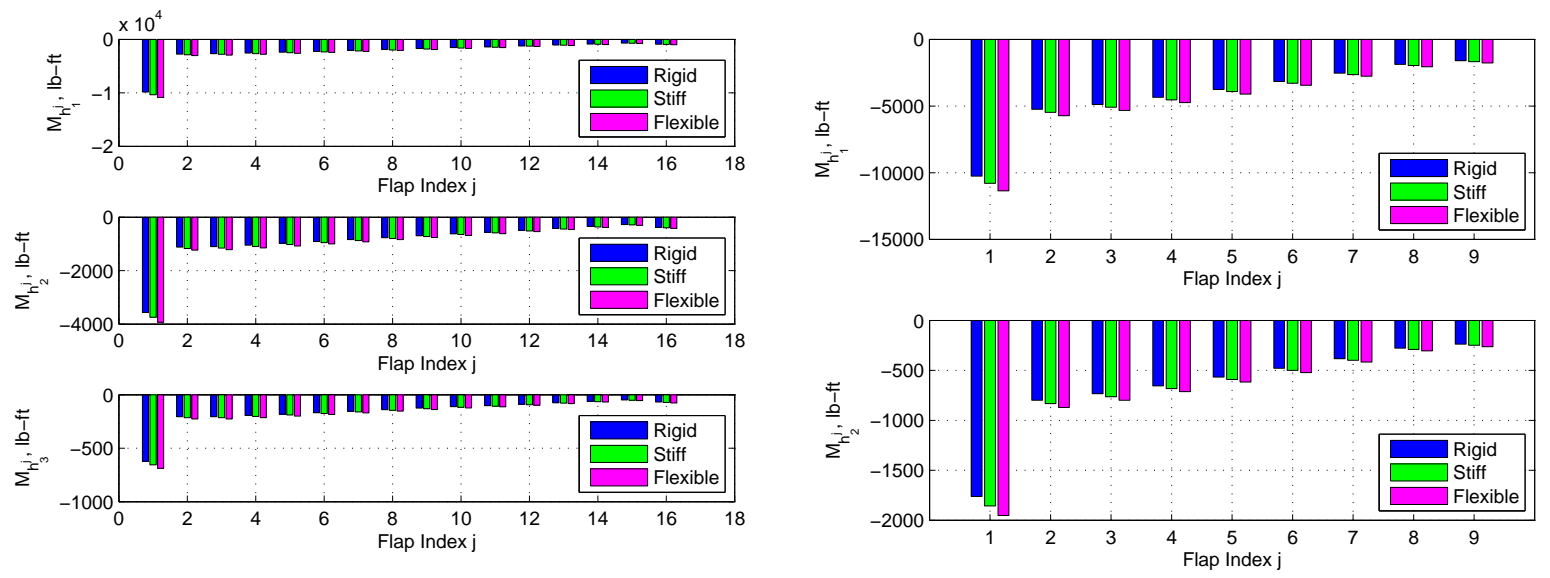

Figure 80. Integrated VCCTEF Hinge Moments $M_{h}$ for Loaded Turn Maneuver at Cruise (VCCTEF configuration \#1, left, VCCTEF configuration \#2, right)

\section{Low-Speed Take-Off}

The results for the three subcases at the low-speed take-off condition of $M=0.228, h=0 \mathrm{ft}$ are presented. For VCCTEF configuration \#1 $\bar{\delta}=25^{\circ}$, and for VCCTEF configuration \#2 $\bar{\delta}=35^{\circ}$.

\section{LOW-Speed TAKe-OfF Steady State}

The static aeroelastic solutions for the rigid, stiff, and flexible wing aircraft configurations at the low-speed steady state flight condition were presented in Table 2. Figure 81 shows the the distributed hinge moment loads $m_{h}(y)$ and the integrated hinge moment loads are shown in Fig. 82.
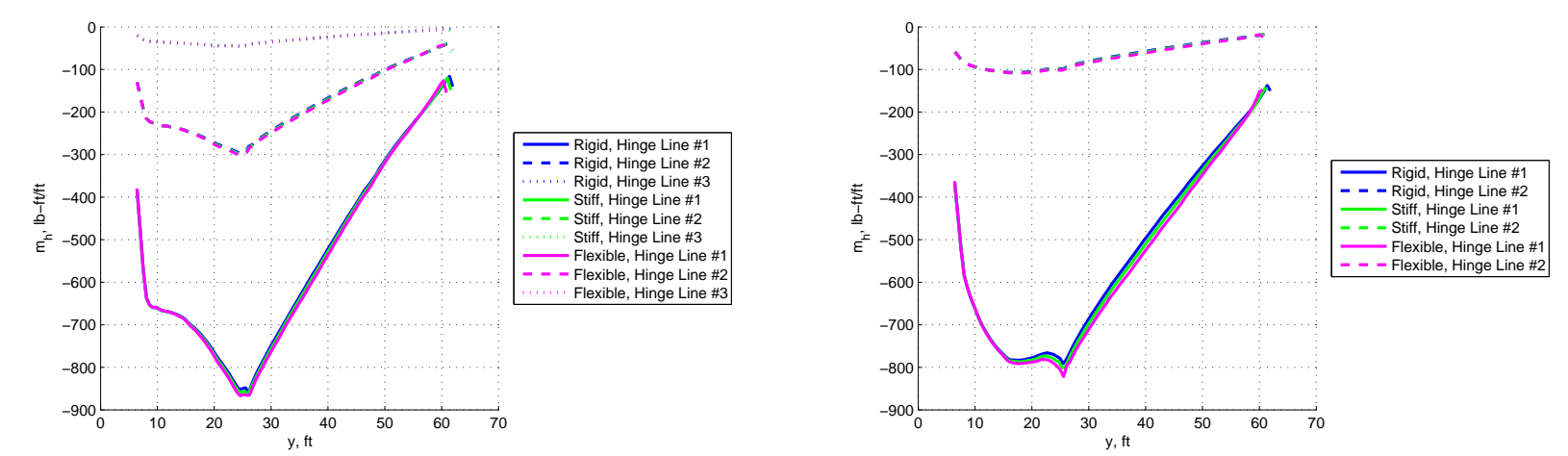

Figure 81. Distributed VCCTEF Hinge Moment Loads $m_{h}(y)$ at Low-Speed Take-Off Steady State (VCCTEF configuration \#1, left, VCCTEF configuration \#2, right) 

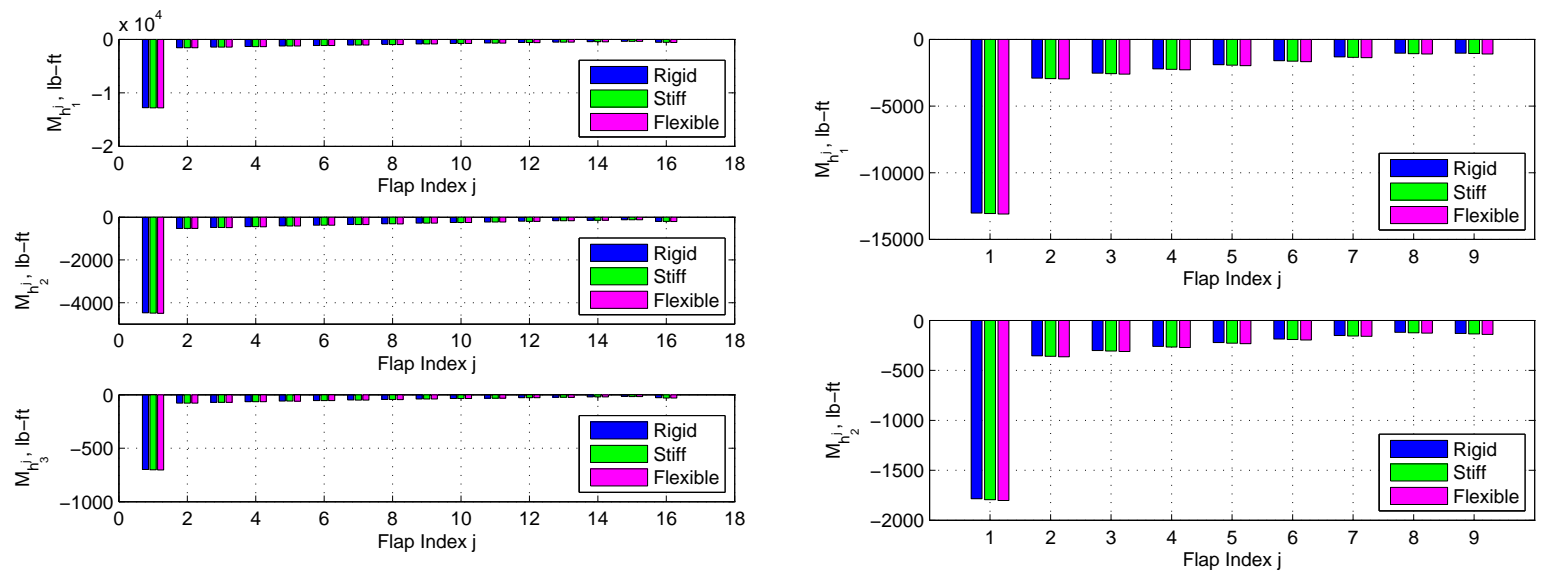

Figure 82. Integrated VCCTEF Hinge Moments $M_{h}$ at Low-Speed Take-Off Steady State (VCCTEF configuration \#1, left, VCCTEF configuration \#2, right)

\section{Low-SPEed TAKe-OFF AT MAXimum LoAD}

The second load case at the low-speed take-off flight condition is considered when the aircraft's angle of attack is increased to the maximum value of $\alpha=10^{\circ}$. The static aeroelastic solutions for the loaded low-speed take-off flight condition are summarized in Table 7.

\begin{tabular}{|c|c|c|c|c|c|c|}
\hline \multicolumn{7}{|c|}{ VCCTEF Configuration \#1 } \\
\hline $\bar{\delta}=25^{\circ}$ & $\alpha(\mathrm{deg})$ & $n$ & $C_{L}$ & $W_{\text {tip }}(\mathrm{ft})$ & $\Theta_{\text {tip }}(\mathrm{deg})$ & $\Delta \phi_{y, t i p}(\mathrm{deg})$ \\
\hline \hline Rigid Wing & 10.000 & 1.089 & 1.70 & - & - & - \\
\hline Stiff Wing & 10.000 & 1.063 & 1.66 & 2.611 & -0.477 & -1.489 \\
\hline Flexible Wing & 10.000 & 1.033 & 1.61 & 5.046 & -0.750 & -3.026 \\
\hline
\end{tabular}

\begin{tabular}{|c|c|c|c|c|c|c|}
\hline \multicolumn{7}{|c|}{ VCCTEF Configuration \#2 } \\
\hline $\bar{\delta}=35^{\circ}$ & $\alpha(\mathrm{deg})$ & $n$ & $C_{L}$ & $W_{\text {tip }}(\mathrm{ft})$ & $\Theta_{\text {tip }}(\mathrm{deg})$ & $\Delta \phi_{y, t i p}(\mathrm{deg})$ \\
\hline \hline Rigid Wing & 10.000 & 1.235 & 1.93 & - & - & - \\
\hline Stiff Wing & 10.000 & 1.208 & 1.89 & 3.327 & -0.491 & -2.015 \\
\hline Flexible Wing & 10.000 & 1.170 & 1.83 & 6.381 & -0.748 & -4.011 \\
\hline
\end{tabular}

Table 7. Static Aeroelastic Solutions for Loaded Low-Speed Take-Off, $M=0.228, h=0$ ft

Figures 83 and 84 show the the distributed hinge moment loads $m_{h}(y)$ and the integrated hinge moment loads for the loaded flight condition. 

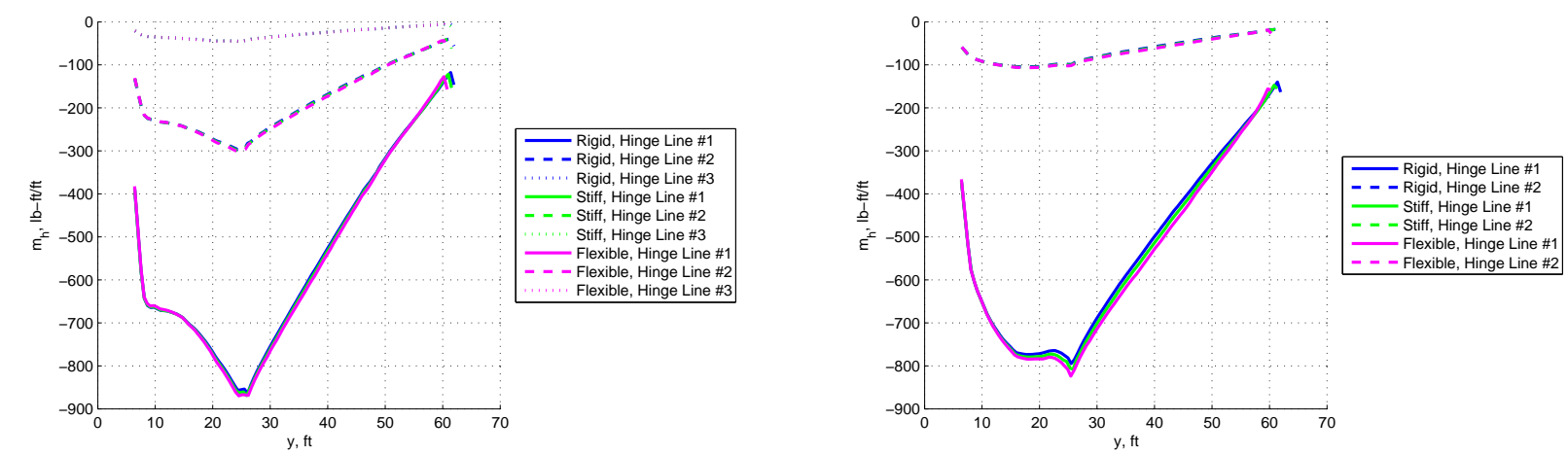

Figure 83. Distributed VCCTEF Hinge Moment Loads $m_{h}(y)$ at Loaded Low-Speed Take-Off (VCCTEF configuration \#1, left, VCCTEF configuration \#2, right)
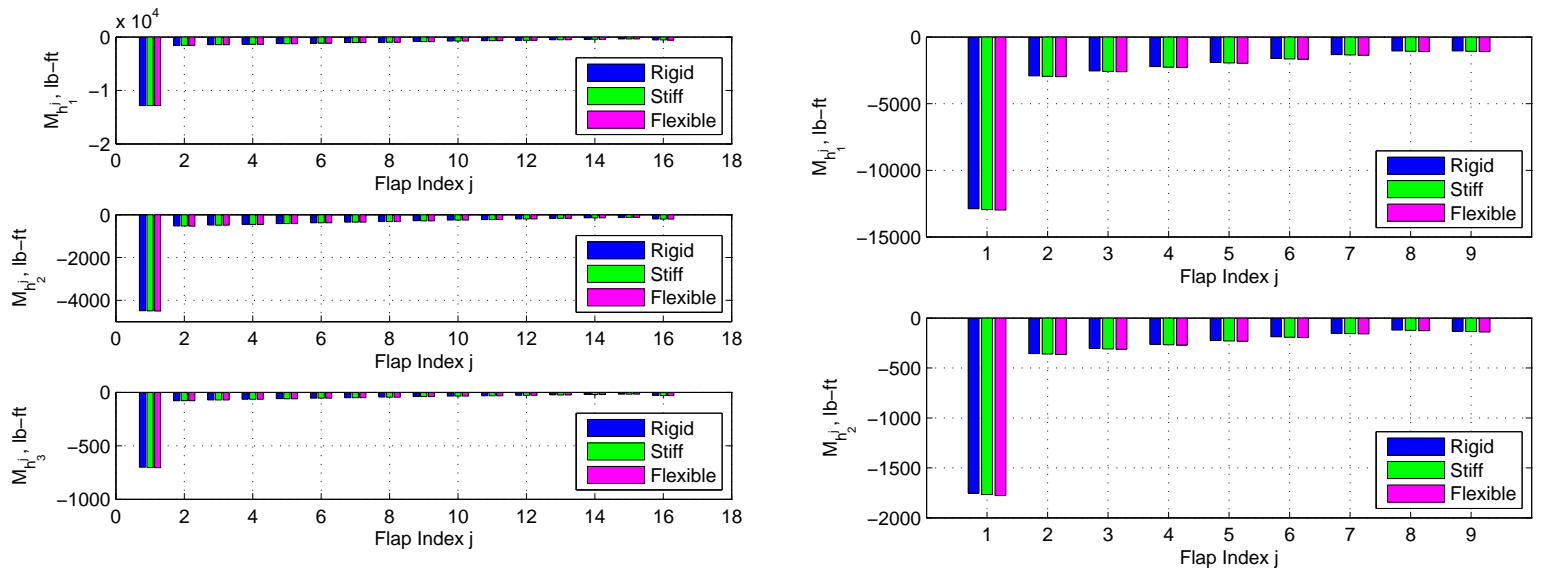

Figure 84. Integrated VCCTEF Hinge Moments $M_{h}$ at Loaded Low-Speed Take-Off (VCCTEF configuration \#1, left, VCCTEF configuration \#2, right)

It is observed that the hinge moment values are barely affected $(<1 \%$ increase on average) when loading the wings from steady state to the loaded cruise condition. This is partly due to the already high angle of attacks for the low-speed take-off flight condition that prevent the maximum load factor from being very high.

\section{Low-SPeEd TAKe-OFF With Limited CoORdinated Turn MANeuver}

The third subcase analyzed for the low-speed take-off flight case models the deflection of the VCCTEF required to conduct a loaded coordinated turn. Table 8 summarizes the aircraft flight configuration, including the maximum load factor, where the stall angle of attack, maneuvering VCCTEF deflection limits, and rudder deflection limits are imposed. 


\begin{tabular}{|c|c|c|c|c|c|c|c|c|c|}
\hline \multicolumn{10}{|c|}{ VCCTEF Configuration \#1 } \\
\hline $\bar{\delta}=25^{\circ}$ & $\alpha(\mathrm{deg})$ & $n$ & $C_{L}$ & $W_{\text {tip }}(\mathrm{ft})$ & $\Theta_{\text {tip }}(\mathrm{deg})$ & $\Delta \phi_{y, t i p}(\mathrm{deg})$ & $C_{l_{\delta_{f}}}$ & $\delta_{f}$ & $\delta r$ \\
\hline \hline Rigid Wing & 10.000 & 1.089 & 1.70 & - & - & - & 0.1331 & 5.629 & 5.567 \\
\hline Stiff Wing & 10.000 & 1.063 & 1.66 & 2.611 & -0.477 & -1.489 & 0.1441 & 3.228 & 3.752 \\
\hline Flexible Wing & 10.000 & 1.033 & 1.61 & 5.046 & -0.750 & -3.026 & 0.1422 & 1.382 & 1.926 \\
\hline
\end{tabular}

\begin{tabular}{|c|c|c|c|c|c|c|c|c|c|}
\hline \multicolumn{10}{|c|}{ VCCTEF Configuration \#2 } \\
\hline $\bar{\delta}=35^{\circ}$ & $\alpha(\mathrm{deg})$ & $n$ & $C_{L}$ & $W_{\text {tip }}(\mathrm{ft})$ & $\Theta_{\text {tip }}(\mathrm{deg})$ & $\Delta \phi_{y, t i p}(\mathrm{deg})$ & $C_{l_{\delta_{f}}}$ & $\delta_{f}$ & $\delta r$ \\
\hline \hline Rigid Wing & 7.855 & 1.150 & 1.79 & - & - & - & 0.1386 & 9.838 & 9.999 \\
\hline Stiff Wing & 8.485 & 1.148 & 1.79 & 3.170 & -0.382 & -2.006 & 0.1442 & 9.686 & 10.001 \\
\hline Flexible Wing & 9.365 & 1.146 & 1.79 & 6.258 & -0.657 & -4.010 & 0.1483 & 9.708 & 10.000 \\
\hline
\end{tabular}

Table 8. Static Aeroelastic Solutions for Loaded Turn Maneuver at Low-Speed Take-Off, $M=0.228, h=0$ ft

The results in Table 8 show that for VCCTEF configuration \#1, the loaded turn maneuver case is limited by stall angle of attack. Thus, the aeroelastic solution for VCCTEF configuration \#1 on the loaded roll maneuver case is the same as the previous maximum loaded subcase. The results for VCCTEF configuration \#2 are rudder deflection limited. The values for the distributed hinge moments loads and the integrated values for each flap section are shown in Figures 85 and 86. Once again, the results represent the positive deflected VCCTEF maneuver sections, and the hinge moment loading for the negative deflected VCCTEF were verified to be less.
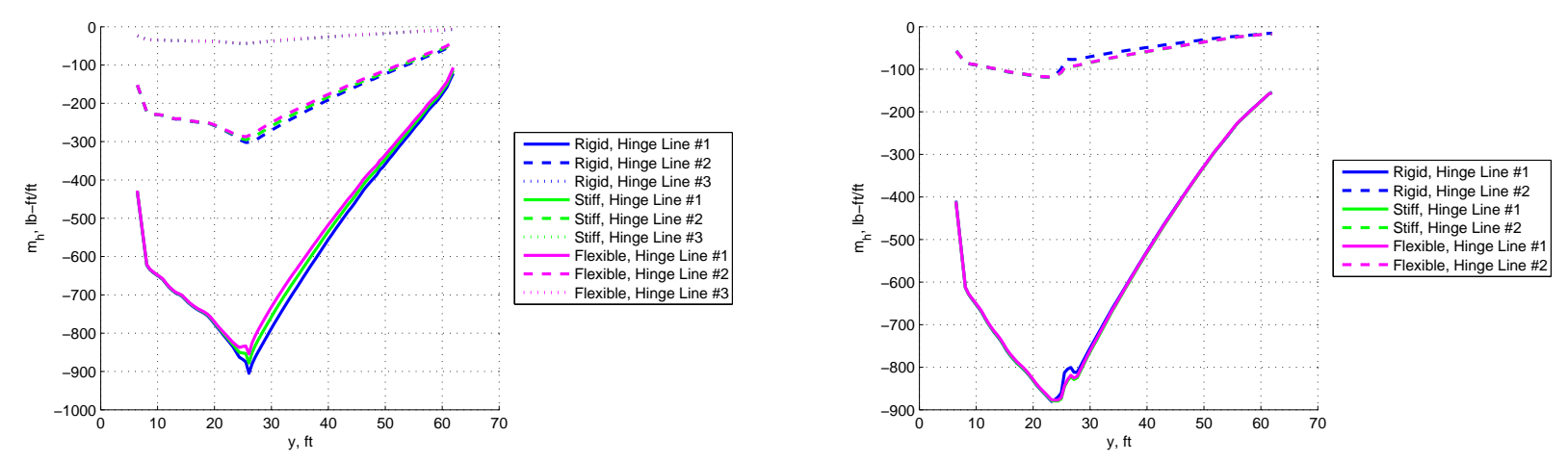

Figure 85. Distributed VCCTEF Hinge Moment Loads $m_{h}(y)$ for Loaded Turn Maneuver at Low-Speed Take-Off (VCCTEF configuration \#1, left, VCCTEF configuration \#2, right) 

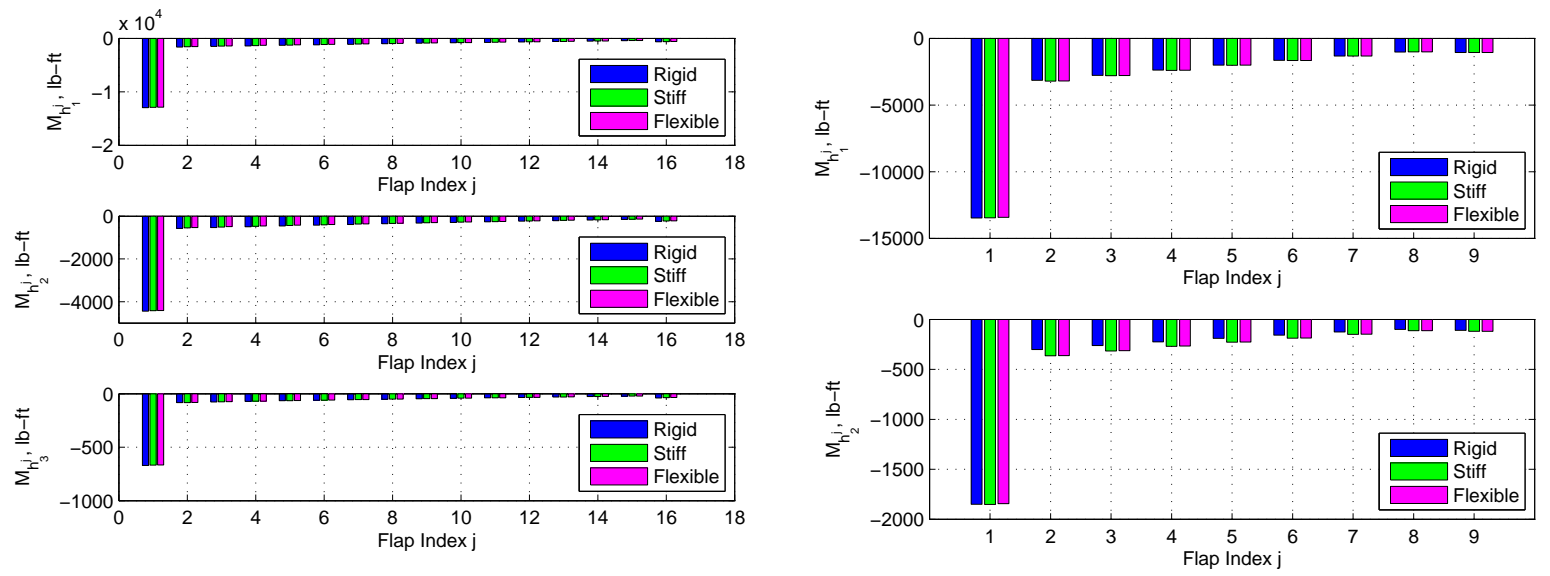

Figure 86. Integrated VCCTEF Hinge Moments $M_{h}$ for Loaded Turn Maneuver at Low-Speed Take-Off (VCCTEF configuration \#1, left, VCCTEF configuration \#2, right)

\section{OVERALl WORST CASE}

The overall worst case hinge moments are determined by comparing the three subcases examined at cruise and the three subcases examined at low-speed take-off. The worst case distributed hinge moment load $m_{h}$ is determined as the maximum value of the hinge moment load at each spanwise section considering the subases examined. It is noted that the critical load does not occur at a single subcase. The data is presented as a function of the normalized wing root to wing tip spanwise variable $\eta=2 \frac{y-y_{\text {root }}}{b^{\prime}-y_{\text {root }}}$ where $b^{\prime}$ is the projected span of the aeroelastically deformed wing in the $y$-direction. The value of $\eta=0$ corresponds to the wing root location and $\eta=1$ corresponds to the wing tip location. Figure 87 is a plot of the worst case distributed hinge moment loads for the rigid, stiff, and flexible wing configurations.
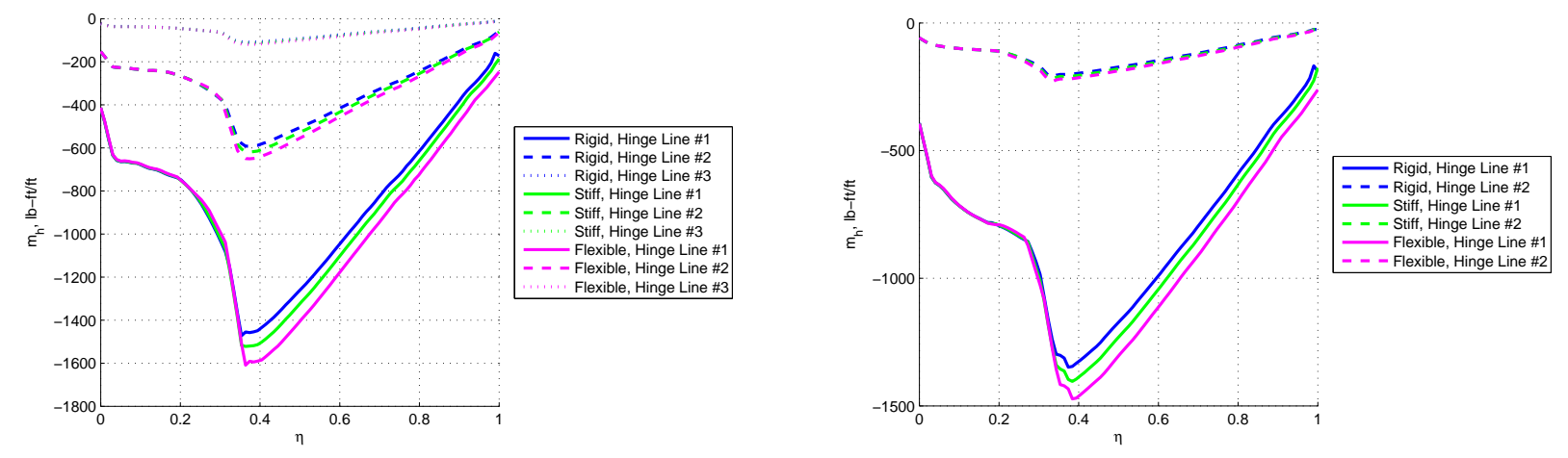

Figure 87. Worst Case Distributed VCCTEF Hinge Moment Loads $m_{h}(\eta)$ (VCCTEF configuration \#1, left, VCCTEF configuration \#2, right)

The worst case integrated hinge moment loads are shown in Fig. 88 for each of the VCCTEF flap sections/segments. The values are kept dimensional with units of $\mathrm{lb}-\mathrm{ft}$ in order to compare results at different dynamic pressure values. 

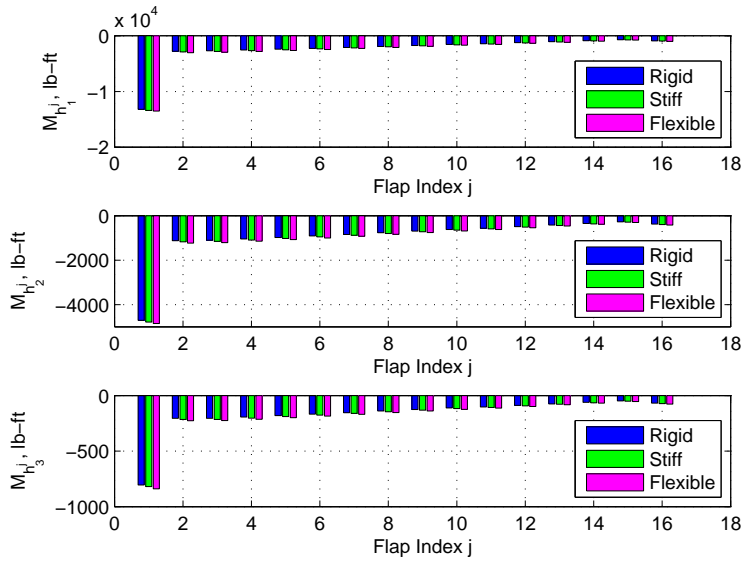
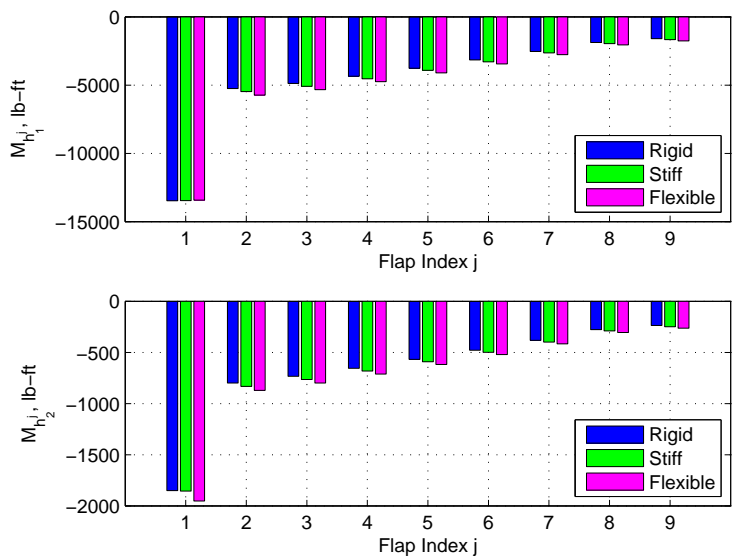

Figure 88. Worst Case Integrated VCCTEF Hinge Moments $M_{h}$ (VCCTEF configuration \#1, left, VCCTEF configuration \#2, right)

The results show that the worst case loads are increased for the VCCTEF as the wing increases in flexibility. This effect is observed to be more prominent on the outboard sections of the wing. Tables 9 and 10 represent the percent increase in the worst case hinge moment for each VCCTEF flap section/segment of the stiff and flexible wings relative to the rigid wing hinge moment values.

\begin{tabular}{|c|c|c|c|}
\hline \multicolumn{4}{|c|}{ VCCTEF Configuration \#1 Stiff } \\
\hline Camber Segment & 1 & 2 & 3 \\
\hline \hline Inner Flap & 1.60 & 1.66 & 1.91 \\
\hline Flap Section 2 & 4.29 & 4.46 & 4.57 \\
\hline Flap Section 3 & 4.34 & 4.51 & 4.55 \\
\hline Flap Section 4 & 4.34 & 4.56 & 4.73 \\
\hline Flap Section 5 & 4.23 & 4.46 & 4.64 \\
\hline Flap Section 6 & 4.16 & 4.36 & 4.74 \\
\hline Flap Section 7 & 4.11 & 4.29 & 4.55 \\
\hline Flap Section 8 & 4.12 & 4.29 & 4.55 \\
\hline Flap Section 9 & 4.17 & 4.36 & 4.69 \\
\hline Flap Section 10 & 4.13 & 4.33 & 4.76 \\
\hline Flap Section 11 & 4.16 & 4.32 & 4.63 \\
\hline Flap Section 12 & 4.24 & 4.42 & 4.82 \\
\hline Flap Section 13 & 4.28 & 4.50 & 5.05 \\
\hline Flap Section 14 & 4.34 & 4.55 & 5.09 \\
\hline Flap Section 15 & 4.46 & 4.69 & 5.37 \\
\hline Flap Section 16 & 4.64 & 4.96 & 5.90 \\
\hline
\end{tabular}

\begin{tabular}{|c|c|c|c|}
\hline \multicolumn{4}{|c|}{ VCCTEF Configuration \#1 Flexible } \\
\hline Camber Segment & 1 & 2 & 3 \\
\hline \hline Inner Flap & 2.45 & 2.93 & 4.19 \\
\hline Flap Section 2 & 8.54 & 8.89 & 9.01 \\
\hline Flap Section 3 & 8.56 & 8.95 & 9.08 \\
\hline Flap Section 4 & 8.59 & 9.06 & 9.31 \\
\hline Flap Section 5 & 8.46 & 8.96 & 9.20 \\
\hline Flap Section 6 & 8.25 & 8.72 & 9.38 \\
\hline Flap Section 7 & 8.20 & 8.60 & 9.22 \\
\hline Flap Section 8 & 8.28 & 8.70 & 9.36 \\
\hline Flap Section 9 & 8.35 & 8.80 & 9.63 \\
\hline Flap Section 10 & 8.34 & 8.78 & 9.66 \\
\hline Flap Section 11 & 8.33 & 8.75 & 9.55 \\
\hline Flap Section 12 & 8.38 & 8.78 & 9.59 \\
\hline Flap Section 13 & 8.50 & 8.94 & 9.93 \\
\hline Flap Section 14 & 8.68 & 9.14 & 10.22 \\
\hline Flap Section 15 & 8.96 & 9.50 & 10.84 \\
\hline Flap Section 16 & 9.17 & 9.79 & 11.42 \\
\hline
\end{tabular}

Table 9. Percent Increase in Worst Case Hinge Moment Relative to Rigid Wing Values for VCCTEF configuration \#1 


\begin{tabular}{|c|c|c|}
\hline \multicolumn{3}{|c|}{ VCCTEF Configuration \#2 Stiff } \\
\hline Camber Segment & 1 & 2 \\
\hline \hline Inner Flap & -0.05 & 0.29 \\
\hline Flap Section 2 & 4.26 & 4.12 \\
\hline Flap Section 3 & 4.28 & 4.13 \\
\hline Flap Section 4 & 4.21 & 4.13 \\
\hline Flap Section 5 & 4.20 & 4.08 \\
\hline Flap Section 6 & 4.29 & 4.26 \\
\hline Flap Section 7 & 4.33 & 4.32 \\
\hline Flap Section 8 & 4.47 & 4.48 \\
\hline Flap Section 9 & 4.74 & 5.03 \\
\hline
\end{tabular}

\begin{tabular}{|c|c|c|}
\hline \multicolumn{3}{|c|}{ VCCTEF Configuration \#2 Flexible } \\
\hline Camber Segment & 1 & 2 \\
\hline \hline Inner Flap & -0.30 & 5.20 \\
\hline Flap Section 2 & 8.41 & 8.14 \\
\hline Flap Section 3 & 8.40 & 8.08 \\
\hline Flap Section 4 & 8.27 & 7.97 \\
\hline Flap Section 5 & 8.31 & 8.02 \\
\hline Flap Section 6 & 8.39 & 8.20 \\
\hline Flap Section 7 & 8.48 & 8.29 \\
\hline Flap Section 8 & 8.86 & 8.94 \\
\hline Flap Section 9 & 9.45 & 10.00 \\
\hline
\end{tabular}

Table 10. Percent Increase in Worst Case Hinge Moment Relative to Rigid Wing Values for VCCTEF configuration \#2

The tables show that the increase in the hinge moment values is roughly proportional to the increase in wing flexibility; the flexible wing, which has about twice the aeroelastic deformation as the stiff wing, shows about twice the percent increase in the worst case hinge moments as the stiff wing. For further insight, Fig. 89 shows color coded diagrams indicating the critical determining load subcases for the VCCTEF flap sections/segments.
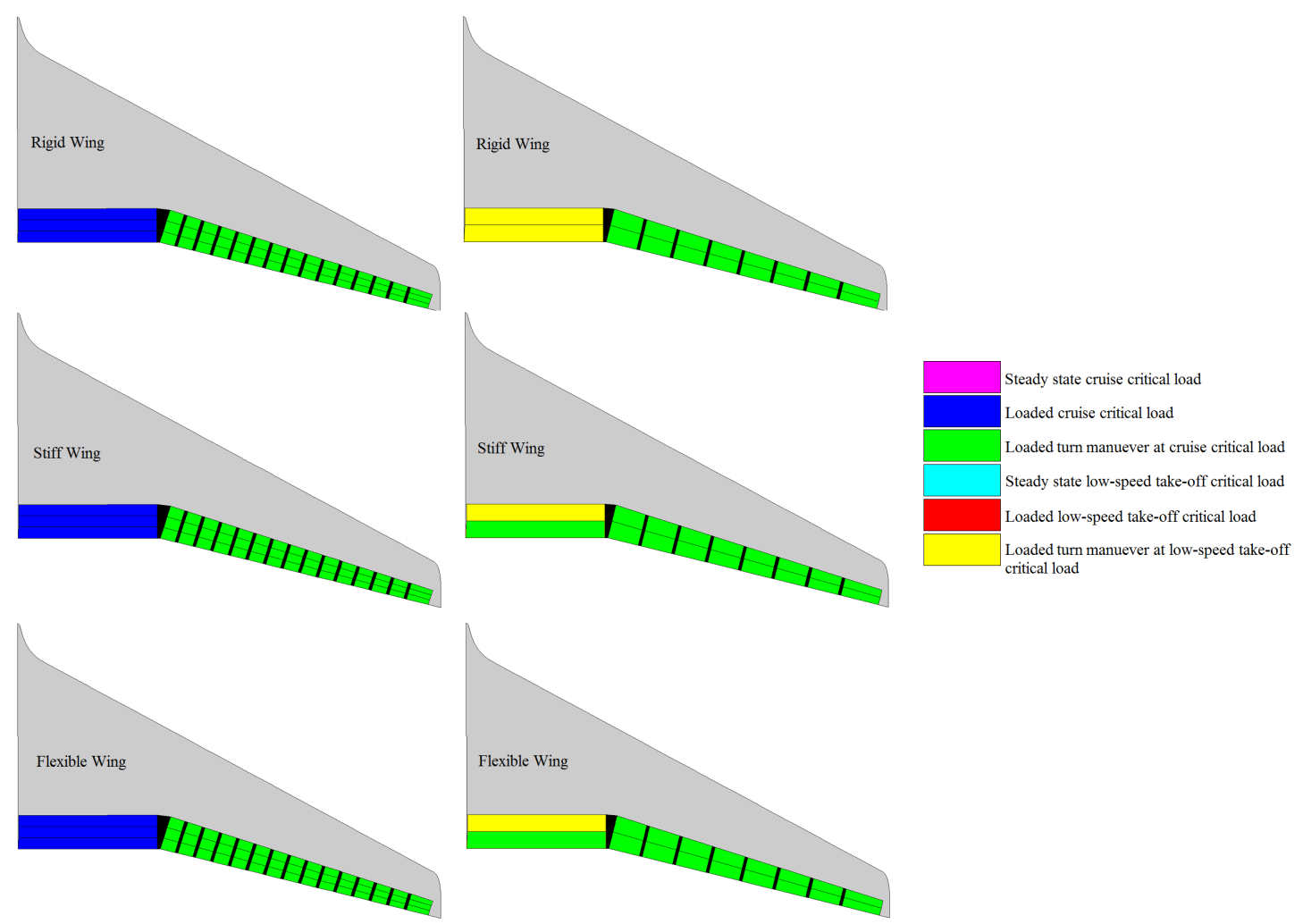

Figure 89. Critical Load Subcases for VCCTEF Sections (VCCTEF configuration \#1, left, VCCTEF configuration \#2, right)

The loaded turn maneuver at cruise is the critical load case for the outboard VCCTEF sections for all the aircraft configurations. For an aircraft with VCCTEF configuration \#1, the inboard section's worst case load is the maximum loaded case at cruise. For VCCTEF configuration \#2, the inboard section's worst case load is the loaded turn maneuver at low-speed take-off, while the second camber segment's is the loaded turn at cruise when wing flexibility is modeled. 


\section{Conclusion}

This paper presents a series of analyses conducted in order to gain insight into aerodynamic loads on the novel VCCTEF control effector when equipped on GTM aircraft with different wing flexibility models. Vortex-lattice method is used as the primary aerodynamic modeling tool, and an aeroelastic model using a coupled vortex-lattice finite-element framework is used to analyze wings with flexibility included. Two VCCTEF configurations are analyzed: VCCTEF configuration \#1 with 16 flap sections each with three camber segments, and VCCTEF configuration \#2 with nine flap sections each with two camber segments. Three wing flexibility models are analyzed: a "rigid" wing model which does not experience aeroelastic deformation, a "stiff" wing model that deforms based on baseline wing mass/stiffness values representative of a current generation aircraft, and a "flexible" wing model that has $50 \%$ reduction in stiffness from the stiff model representative of a future generation aircraft. Two primary flight conditions are considered for analysis: cruise and low-speed take-off. Aeroelastic solutions for the flight conditions considered are presented.

Two primary types of analyses are conducted on the aircraft configurations. The first type of analysis is control derivative analysis where the sensitivity of the aerodynamic loads to perturbations of the VCCTEF flap sections are determined. A methodology is presented utilizing vortex-lattice, and results are presented for rigid, stiff, and flexible wing platforms at the cruise and low-speed take-off conditions. For the cruise flight condition, a nominal VCCTEF flap deflection is considered of $\bar{\delta}=0$ or no VCCTEF deflection. For the low-speed take-off flight condition, an analysis is conducted in order to determine the nominal flap deflection $\bar{\delta}$ at which the VCCTEF has roll control authority similar to that of a conventional aileron system. For VCCTEF configuration \#1, the nominal flap deflection is determined to be $\bar{\delta}=25^{\circ}$. For VCCTEF configuration \#2, the nominal flap deflection is determined to be $\bar{\delta}=35^{\circ}$. With respect to VCCTEF flap deflections, plots of sectional aerodynamic derivatives are presented as well as total aircraft derivatives for the cruise and the low-speed take-off conditions about the nominal flap deflection values. The aerodynamic load and moment derivatives with respect to the fast actuated sections of the VCCTEF utilized for maneuvering are also tabulated. The results demonstrate trends when including aeroelastic modeling, where the lift coefficient derivatives $C_{L_{\delta}}$ and roll moment coefficient derivatives $C_{l_{\delta}}$ experience slight increases as wing flexibility is increased.

The second type of analysis conducted is hinge moment. A methodology is presented that utilizes vortex-lattice in order to determine the aerodynamic loading on VCCTEF flap sections along the hinge lines that can be integrated to determine the total load over a VCCTEF flap section/segment. For both the cruise and low-speed take-off flight conditions, hinge moment analyses are conducted at various different subcases. For both flight conditions, the hinge moments at the steady state flight condition, the hinge moments at a max loaded flight condition without VCCTEF deflection, and the hinge moments at a loaded coordinated turn flight condition with VCCTEF deflection are determined. For the max loaded flight conditions, $\alpha_{\text {stall }}$ is considered to be $\alpha_{\text {stall }}=12^{\circ}$ at cruise and $\alpha_{\text {stall }}=10^{\circ}$ at low-speed take off. For the loaded flight condition while executing coordinated turn, the aircraft maintains the stall angle of attack restriction as well as imposed antisymmetric VCCTEF maneuver section deflection limits at $\delta_{f, \max }=30^{\circ}$ and rudder deflection limits at $\delta r_{\max }=10^{\circ}$. For cruise, the maximum load condition is at $n=2.5$ for the rigid and stiff wing models while the $\alpha_{\text {stall }}$ is the limit for the flexible wing model. For the turn maneuver loaded flight condition, the models are all restricted by the rudder deflection. For low-speed take-off, $\alpha_{\text {stall }}$ is the maximum load condition, whereas when conducting the coordinated turn, VCCTEF configuration \#1 is limited by $\alpha_{\text {stall }}$ but VCCTEF configuration \#2 is limited by rudder deflection. The estimated worst case hinge moments on the three wing configurations for both VCCTEF configurations is determined by examining the largest hinge moments of the total six subcases (three subcases for cruise, three subcases for low-speed take-off). For both VCCTEF configurations, the outboard VCCTEF flap sections/segment's critical hinge moment loads are experienced during the loaded turn maneuver at cruise. For VCCTEF configuration \#1, the inner flap's critical hinge moment loads are experienced at the maximum load condition. For VCCTEF configuration \#2, the inner flap experiences the maximum load during the loaded turn maneuver at low-speed take-off. Hinge moments are affected by flexibility of the wing. For VCCTEF configuration \#1, the stiff wing experiences a maximum increase of 5.90\% in hinge moment load relative to the rigid wing and the flexible wing experiences a maximum increase of $11.42 \%$. For VCCTEF configuration \#2, the stiff wing experiences a maximum increase of $5.03 \%$ in hinge moment load relative to the rigid wing and the flexible wing experiences a maximum increase of $10.00 \%$. Thus, the observed trend is that as the wing flexibility increases, the maximum worst case hinge moments increase roughly proportionally to the decrease in stiffness.

The results of the control derivative analyses and the hinge moment analyses represent first-order aerodynamic analyses of the loading that the VCCTEF control effectors experience on conventional commercial aircraft configurations where wing aeroelasticity is considered. As a tool, vortex-lattice is only able to conduct quasi-steady aerodynamic analysis. Further work with regards to control derivatives involves incorporating unsteady aerodynamics and 
the effect of the rate of change of VCCTEF deflections on the aircraft sectional and total aerodynamic loads. Further refinements can be incorporated into the vortex-lattice modeling of the GTM as well, where the engine nacelles can be added by utilizing fusiform modeling. Asymmetric modeling of the aircraft can also be investigated. These improvements and future work also affects the hinge moment analysis, but further considerations of additional flight conditions and subcases can be considered. This work considers only the cruise and the low-speed take-off flight conditions, but additional points in the flight envelope can be included as well as other maximum load cases, such a maximum roll rate case. Higher fidelity aerodynamic modeling tools can be used to approximate loads at the lift-off condition or landing condition where viscous effects dominate. As these further extensions of this aerodynamic modeling work are being conducted, the results from this study can be utilized in flight dynamic, stability, and control modeling that involves the VCCTEF. Conventional rigid body aircraft flight dynamic models can incorporate the aircraft control derivative results while coupling with flexible wing models can utilize the spanwise derivative and extended hinge moment results. Hinge moment results can also be used by design and system engineers to size the actuation systems for wings designs with VCCTEF.

\section{Acknowledgments}

The authors would like to thank the Fixed Wing Project under the Fundamental Aeronautics Program of NASA Aeronautics Research Mission Directorate (ARMD) for funding support of this work. The authors also acknowledge Boeing Research and Technology for their collaboration with NASA on this work.

\section{References}

${ }^{1}$ Nguyen, N., "Elastically Shaped Future Air Vehicle Concept," NASA Innovation Fund Award 2010 Report, http://ntrs.nasa.gov/archive/nasa/casi.ntrs.nasa.gov/20110023698_2011024909.pdf, Submitted to NASA Innovative Partnerships Program, October 2010.

${ }^{2}$ Nguyen, N., Trinh, K., Reynolds, K., Kless, J., Aftosmis, M., Urnes, J., and Ippolito C., "Elastically Shaped Wing Optimization and Aircraft Concept for Improved Cruise Efficiency," AIAA Aerospace Sciences Meeting, AIAA-2013-0141, January 2013.

${ }^{3}$ Boeing Report No. 2010X0015, "Development of Variable Camber Continuous Trailing Edge Flap System," October 4, 2012.

${ }^{4}$ Urnes, J., Nguyen N., Ippolito, C., Totah, J., Trinh, K., and Ting, E., "A Mission Adaptive Variable Camber Flap Control System to Optimize High Lift and Cruise Lift to Drag Ratios of Future N+3 Transport Aircraft,” AIAA Aerospace Sciences meeting, AIAA-2013-0214, January 2013.

${ }^{5}$ Jordan, T. L., Langford, W. M., Belcastro, C. M., Foster, J. M., Shah, G. H., Howland, G., and Kidd, R., "Development of a Dynamically Scaled Generic Transport Model Testbest for Flight Research Experiments," AUVSI Unmanned Unlimited, Arlington, VA, 2004.

${ }^{6}$ Nguyen, N., Ting, E., Nguyen, D., Dao, T., Trinh, K., "Coupled Vortex-Lattice Flight Dynamic Model with Aeroelastic Finite-Element Model of Flexible Wing Transport Aircraft with Variable Camber Continuous Trailing Edge Flap for Drag Reduction", AIAA Atmospheric Flight Mechanics (AFM) Conference, AIAA-2013-4746, August 2013

${ }^{7}$ Ting, E., Nguyen, N., Trinh, K., "Static Aeroelastic and Longitudinal Trim Model of Flexible Wing Aircraft Using Finite-Element VortexLattice Coupled Solution”, AIAA Structures, Structural Dynamics, and Materials Conference, AIAA-2014-0837, January 2014.

${ }^{8}$ Anderson, J. D., Fundamentals of Aerodynamics, McGraw-Hill Higher Education, 2001.

${ }^{9}$ Miranda, L.R., Elliot, R.D., and Baker, W.M., "A Generalized Vortex Lattice Method for Subsonic and Supersonic Flow Applications," NASA CR-2865, 1977.

${ }^{10}$ Nguyen, N., Ting, E., Swei, S., Ishihara, A., "Distributed Parameter Optimal Control by Adjoint Aeroelastic Differential Operators for Mode Suppression Control”, AIAA Guidance, Navigation, and Control (GNC) Conference, AIAA-2013-4859, August 2013.

${ }^{11}$ Nguyen, N., Nelson, A., and Pulliam, T., "Damage Adaptive Control System Research Report," Internal NASA Report, April 2006.

${ }^{12}$ Aftosmis, M.J., Berger, M.J., and Melton, J.E., "Robust and Efficient CartesianMesh Generation for Component- Based Geometry,” AIAA Journal, Vol. 36, No. 6, 1998, pp. 953-960.

${ }^{13}$ Lebofsky, S., Ting, E., Nguyen, N., "Aeroelastic Modeling and Drag Optimization of Aircraft Wing with Variable Camber Continuous Trailing Edge Flap,” AIAA Applied Aerodynamics Conference, AIAA-2014-2443, June 2014.

${ }^{14}$ Houbolt, J. C and Brooks, G. W., "Differential Equations of Motion for Combined Flapwise Bending, Chordwise Bending, and Torsion of Twisted Nonuniform Rotor Blades," NACA Technical Note 3905, February 1957.

${ }^{15}$ Meirovitch, L., Fundamentals of Vibrations, McGraw-Hill Higher Education, 2001.

${ }^{16}$ Hodges, D.H. and Pierce, G.A., Introduction to Structural Dynamics and Aeroelasticity, Cambridge University Press, 2002.

${ }^{17}$ Hughes, T., The Finite Element Method Linear Static and Dynamic Finite Element Analysis, Prentice Hall, Inc., 1987.

${ }^{18}$ Craig, Jr., R.R. and Kurdila, A.J., Fundamentals of Structural Dynamics, Second Edition, John Wiley \& Sons, Inc., 2006.

${ }^{19}$ Nguyen, N., Trinh, K., Frost S., and Reynolds, K., "Coupled Aeroelastic Vortex Lattice Modeling of Flexible Aircraft," AIAA Applied Aerodynamics Conference, AIAA-2011-3021, June 2011.

${ }^{20}$ Nguyen, N., Trinh, K., Nguyen, D., Tuzcu, I., "Nonlinear Aeroelasticty of Flexible Wing Structure Coupled with Aircraft Flight Dynamics," AIAA Structures, Structural Dynamics, and Materials Conference, AIAA-2012-1792, April 2012.

${ }^{21}$ Martin, G. L., "Paneling Techniques for Use with the Vorlax Computer Program," NASA Contractor Report NASA CR-145364, 1978. 